\title{
REGIOCONTROLLED COUPLING OF ALKYNES AND DIPOLAR REAGENTS: IRON-MEDIATED [3+2] CYCLOADDITIONS REVISITED
}

Jin Zhu, Austin C. Durham, Yidong Wang, James C. Corcoran, Xiao-Dong Zuo, Steven J. Geib and Yi-Ming Wang

Department of Chemistry, University of Pittsburgh, Pittsburgh, PA, 15260 


\section{Contents}

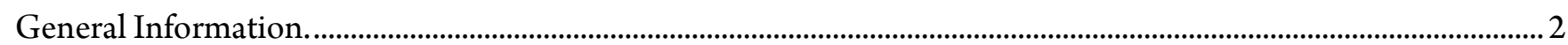

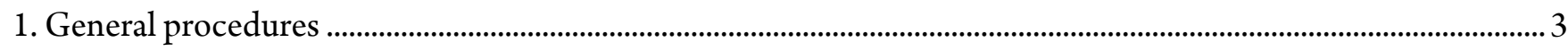

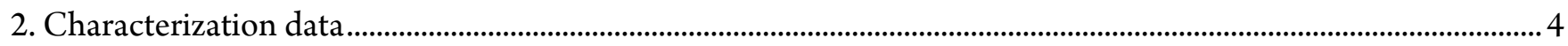

2.1 Synthesis of iron complexes ........................................................................................................................

2.2 Demetallation reactions of iron complex.................................................................................................................

2.3 Synthesis of $\mathrm{N}$-arylpyrazoles .............................................................................................................................

2.4 Synthesis of $\mathrm{N}$-acylpyrazole 14 ..........................................................................................................................11

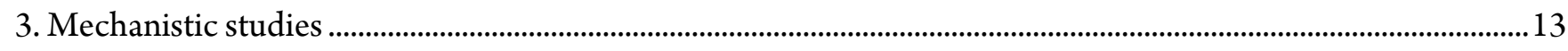

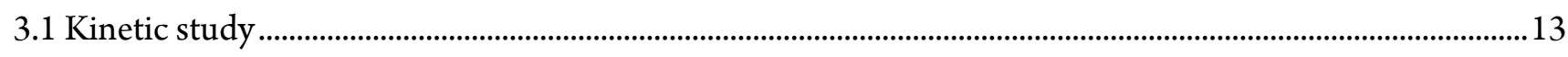

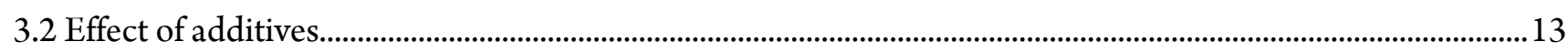

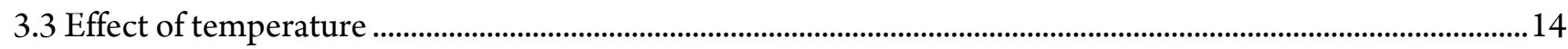

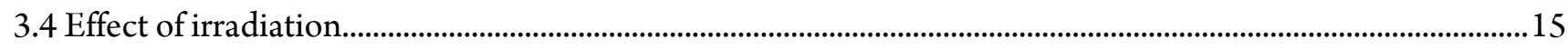

3.5 Effect of added diazonium salt ............................................................................................................................20

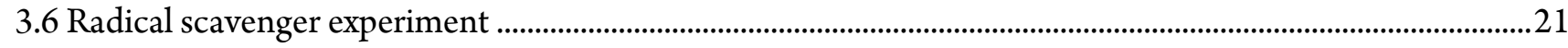

3.7 Effect of added silver iodide or silver $(0)$..............................................................................................................21

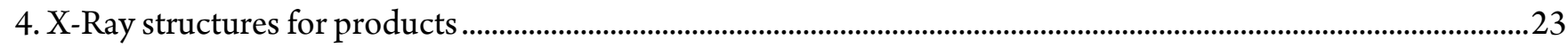

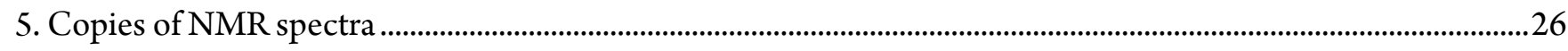

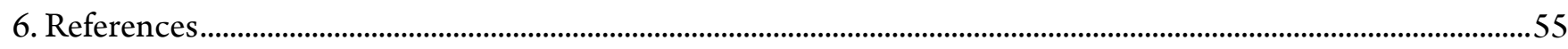




\section{General Information.}

Anhydrous solvents were purchased from Acros (AcroSeal packaging), Sigma Aldrich (Sure/Seal packaging), and Frontier Scientific (J\&KSeal packaging), respectively, and were transferred into an argon-filled glovebox and used as received. All reagents were purchased from Oakwood, Acros, Alfa Aesar, or Sigma Aldrich and used as received. Compounds were purified by flash column chromatography using SiliCycle SiliaFlash ${ }^{\circledast}$ F60 silica gel, unless otherwise indicated. Thin-layer chromatography (TLC) was performed on Silicycle $250 \mu \mathrm{m}$ (analytical) or $1000 \mu \mathrm{m}$ (preparative) silica gel plates. Compounds were visualized by irradiation with UV light, or by staining with iodine/silica gel, potassium permanganate, or phosphomolybdic acid (PMA). Yields refer to isolated compounds, unless otherwise indicated. All compounds (starting materials and products) were characterized by ${ }^{1} \mathrm{H} \mathrm{NMR},{ }^{19} \mathrm{~F} \mathrm{NMR},{ }^{13} \mathrm{C} \mathrm{NMR}$, low-resolution mass spectrometry and highresolution mass spectrometry. ${ }^{1} \mathrm{H}$ NMR spectra were recorded on Bruker $400 \mathrm{MHz}, 500 \mathrm{MHz}$, or $600 \mathrm{MHz}$ spectrometer and are referenced relative to residual $\mathrm{CDCl}_{3}$ proton signals at $\delta 7.26 \mathrm{ppm}, \mathrm{CD}_{2} \mathrm{Cl}_{2}$ at $\delta 5.33 \mathrm{ppm}, \mathrm{C}_{6} \mathrm{D}_{6}$ at $\delta 7.15 \mathrm{ppm}$. Data for ${ }^{1} \mathrm{H}$ and ${ }^{19} \mathrm{~F}$ NMR are reported as follows: chemical shift $(\delta \mathrm{ppm})$, multiplicity $(\mathrm{s}=$ singlet, $\mathrm{d}=$ doublet, $\mathrm{t}=$ triplet, $\mathrm{q}=$ quartet, $\mathrm{m}=$ multiplet, $\mathrm{ap}=$ apparent $)$, integration, and coupling constant $(\mathrm{Hz}) \cdot{ }^{13} \mathrm{C}$ NMR spectra were recorded on a Bruker $400 \mathrm{MHz}, 500 \mathrm{MHz}$, or $600 \mathrm{MHz}$ spectrometer and are referenced to $\mathrm{CDCl}_{3}$ at $\delta 77.16 \mathrm{ppm}, \mathrm{CD}_{2} \mathrm{Cl}_{2}$ at $\delta 53.84$ $\mathrm{ppm}, \mathrm{C}_{6} \mathrm{D}_{6}$ at $\delta 128.39 \mathrm{ppm}$. Unless otherwise stated, the ${ }^{13} \mathrm{C}$ NMR spectra were obtained with ${ }^{1} \mathrm{H}$ decoupling. Data for ${ }^{13} \mathrm{C}$ NMR are reported in terms of chemical shift and multiplicity where appropriate. High Resolution Mass spectra were obtained on a Bruker Daltonics, Inc. APEXIII 7.0 TESLA FTMS instrument (ESI) or Waters Micromass GCT Premier instrument (EI). IR spectra were recorded on a Thermo Scientific Nicolet iS5 spectrometer (iD5 ATR, diamond) and are reported in terms of frequency of absorption $\left(\mathrm{cm}^{-1}\right)$. Elemental analyses were performed by Atlantic Microlabs Inc., Norcross, GA. 


\section{General procedures}

General procedure A for the synthesis of $\eta^{2}$-alkynyl cyclopentadienyliron dicarbonyl tetrafluoroborate complexes or $\eta^{2}$-alkynyl pentamethylcyclopentadienyliron dicarbonyl tetrafluoroborate complexes

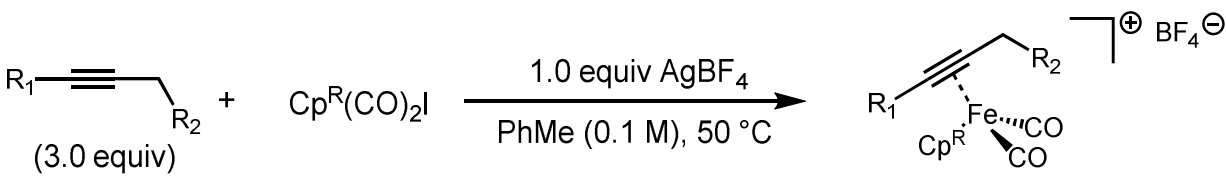

In an argon-filled glovebox, a flame-dried round-bottom flask was charged with $\mathrm{Cp}^{*} \mathrm{Fe}(\mathrm{CO})_{2} \mathrm{I}$ or $\mathrm{CpFe}(\mathrm{CO})_{2} \mathrm{I}(1.0 \mathrm{mmol}$, 1.0 equiv), $\operatorname{AgBF}_{4}$ (1.0 mmol, 1.0 equiv) and a stir bar. A solution of alkyne (3.0 mmol, 3.0 equiv) in dry toluene $(10 \mathrm{~mL})$ was added sequentially, and the obtained suspension was stirred at $50{ }^{\circ} \mathrm{C}$ until the supernatant became pale orange (typically $3 \mathrm{~h}$ ). The reaction mixture was cooled to room temperature and filtered through a pad of Celite. The filter cake was rinsed with $\mathrm{CH}_{2} \mathrm{Cl}_{2}$ until the filtrate was colorless. The desired iron complex was collected as dark yellow solids upon concentration in vacuo. The crude mixture was recrystallized from 1:1 of $\mathrm{CH}_{2} \mathrm{Cl}_{2}$ and $\mathrm{Et}_{2} \mathrm{O}$. The complex was stored at dark without apparent degradation for several days.

General procedure $\mathbf{B}$ for the synthesis of $\eta^{1}$-allenyl cyclopentadienyliron dicarbonyl complexes or $\eta^{1}$-allenyl pentamethylcyclopentadienyliron dicarbonyl complexes

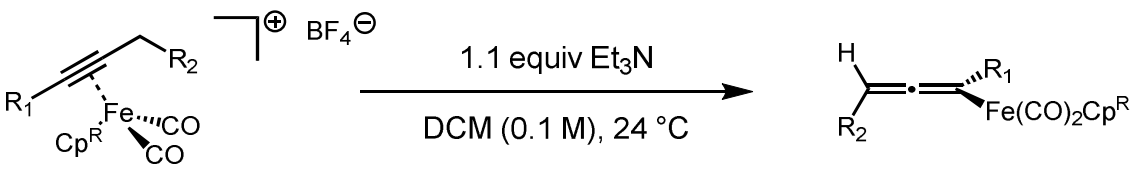

To a solution of $\eta^{2}$-alkynyl cyclopentadienyliron dicarbonyl tetrafluoroborate complex $(0.20 \mathrm{mmol}, 1.0$ equiv $)$ in dry $\mathrm{CH}_{2} \mathrm{Cl}_{2}(2 \mathrm{~mL})$ was added $\mathrm{Et}_{3} \mathrm{~N}(0.22 \mathrm{mmol}, 1.1$ equiv $)$ under air. The mixture was stirred at room temperature for $1 \mathrm{~min}$. The solvent was removed under reduced pressure for another $2 \mathrm{~min}$. $\mathrm{Et}_{2} \mathrm{O}$ was added into the crude solid and the suspension was filtered through a pad of Celite and washed with additional $\mathrm{Et}_{2} \mathrm{O}$. The filtrate was concentrated to give the product as yellow oily solid. After removal of residual $\mathrm{Et}_{2} \mathrm{O}$ under vacuum, the allenyliron complex was sufficiently pure and was used for the next coupling step without further purification.

General procedure $\mathbf{C}$ for the Synthesis of $\mathrm{N}$-arylpyrazoles

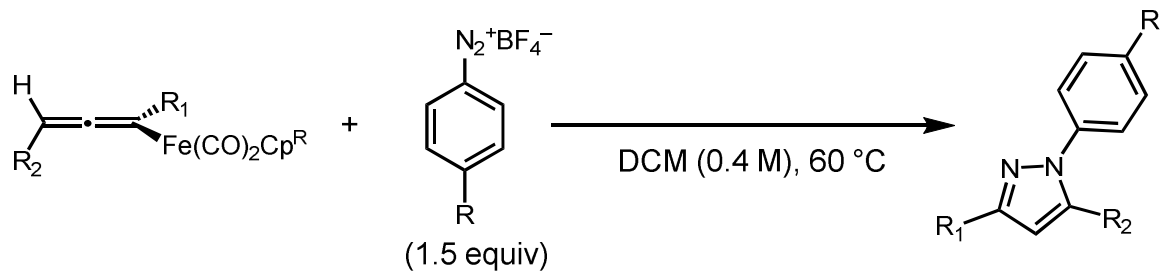

In an argon-filled glovebox, a sealed reaction tube equipped with a magnetic stir bar was charged with diazonium tetrafluoroborate ( $0.30 \mathrm{mmol}, 1.5$ equiv). A solution of freshly-prepared allenyliron complex $\left(0.20 \mathrm{mmol}, 1.0\right.$ equiv) in $\mathrm{CH}_{2} \mathrm{Cl}_{2}$ $(0.5 \mathrm{~mL})$ was added in rapid succession. The reaction tube was sealed and moved from the glovebox. The reaction tube was placed in an oil bath, which was preheated to $60^{\circ} \mathrm{C}$. After completion of the reaction (typically $12 \mathrm{~h}$ ), the reaction mixture was cooled to room temperature. The crude mixture was concentrated in vacuo and purified by flash column chromatography to provide the desired product. 


\section{Characterization data}

\subsection{Synthesis of iron complexes}

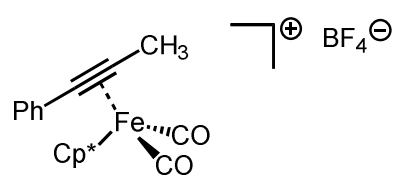

$1-I$

Iron complex 1-I. Prepared following General Procedure A using 1-phenyl-1-propyne (0.4 mL, $3.0 \mathrm{mmol}, 3.0$ equiv), $\mathrm{Cp}^{*} \mathrm{Fe}(\mathrm{CO})_{2} \mathrm{I}\left(374 \mathrm{mg}, 1.0 \mathrm{mmol}, 1.0\right.$ equiv), and $\mathrm{AgBF}_{4}(195 \mathrm{mg}, 1.0 \mathrm{mmol}, 1.0$ equiv). The reaction mixture was heated at $50{ }^{\circ} \mathrm{C}$ for $3 \mathrm{~h}$, filtered through a pad of Celite, and washed with $\mathrm{CH}_{2} \mathrm{Cl}_{2}(25 \mathrm{~mL})$. The solvent was evaporated to obtain the crude product as a dark orange solid. The crude solid was kept under vacuum overnight, then dissolved in $\mathrm{CH}_{2} \mathrm{Cl}_{2}$ and filtered to remove residual impurities. After removal of solvent, the desired product 1-I was recrystallized from $\mathrm{Et}_{2} \mathrm{O}$ / $\mathrm{CH}_{2} \mathrm{Cl}_{2}(1: 1 \mathrm{v} / \mathrm{v})$ as a bright yellow solid. Note: For the preparation of $\mathbf{1 a}$ in high purity, the storage of the crude solid overnight before filtration and recrystallization should not be omitted. Yield: $358 \mathrm{mg}, 79 \% .{ }^{1} \mathbf{H} \mathbf{~ N M R}\left(400 \mathrm{MHz}, \mathrm{CD}_{2} \mathrm{Cl}_{2}\right)$ $\delta$ 7.61-7.59 (m, 2H), 7.54-7.53 (m, 3H), $2.67(\mathrm{~s}, 3 \mathrm{H}), 1.88(\mathrm{~s}, 15 \mathrm{H}) .{ }^{19} \mathbf{F}$ NMR $\left(376 \mathrm{MHz}, \mathrm{CD}_{2} \mathrm{Cl}_{2}\right) \delta-152.79,-152.84$. ${ }^{13} \mathrm{C}$ NMR $\left(100 \mathrm{MHz}, \mathrm{CD}_{2} \mathrm{Cl}_{2}\right) \delta 210.9(\mathrm{CO}), 132.3(\mathrm{Ar}), 130.4(\mathrm{Ar}), 129.5(\mathrm{Ar}), 121.6(\mathrm{Ar}), 103.0\left(\underline{\mathrm{C}}_{5} \mathrm{Me}_{5}\right), 64.2(\mathrm{C} \equiv \mathrm{C})$, $58.6(\mathrm{C} \equiv \mathrm{C}), 9.5\left(\mathrm{C}_{5} \underline{\mathrm{Me}}_{5}\right)$ (one methyl carbon signal missing due to overlap). IR (ATR): $v(\mathrm{CO})=2049,2009 \mathrm{~cm}^{-1}$. HRMS (ESI) calcd. for $\mathrm{C}_{21} \mathrm{H}_{23} \mathrm{O}_{2} \mathrm{Fe}\left[\mathrm{M}-\mathrm{BF}_{4}\right]^{+}: 363.1042$, found 363.1039. EA calcd. for $\mathrm{C}_{21} \mathrm{H}_{23} \mathrm{O}_{2} \mathrm{FeBF}$ : $\mathrm{C}$ 56.04, $\mathrm{H}$ 5.15, found: C 55.74, H 5.12 .

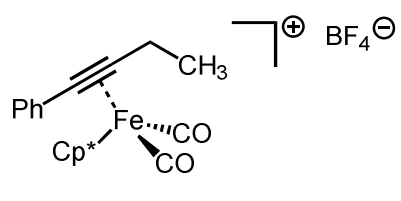

7-I

Iron complex 7-I. Prepared following a literature procedure. ${ }^{1}$

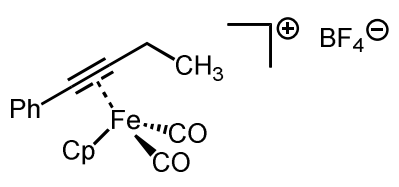

7-II

Iron complex 7-II. Prepared following General Procedure A using 1-phenyl-1-butyne (0.85 mL, $6.0 \mathrm{mmol}, 3.0$ equiv), $\mathrm{CpFe}(\mathrm{CO})_{2} \mathrm{I}\left(608 \mathrm{mg}, 2.0 \mathrm{mmol}, 1.0\right.$ equiv), and $\mathrm{AgBF}_{4}(390 \mathrm{mg}, 2.0 \mathrm{mmol}, 1.0$ equiv). The reaction mixture was heated at $50{ }^{\circ} \mathrm{C}$ for $3 \mathrm{~h}$, filtered through a pad of Celite, and washed with $\mathrm{CH}_{2} \mathrm{Cl}_{2}(50 \mathrm{~mL})$. The solvent was evaporated to obtain the desired product as a dark orange solid. The crude solid was recrystallized from $\mathrm{Et}_{2} \mathrm{O} / \mathrm{CH}_{2} \mathrm{Cl}_{2}(1: 1 \mathrm{v} / \mathrm{v})$ to afford 7-II as a bright orange solid. Yield: $538 \mathrm{mg}, 68 \%$. ${ }^{1} \mathbf{H}$ NMR $\left(400 \mathrm{MHz}, \mathrm{CD}_{2} \mathrm{Cl}_{2}\right) \delta 7.65$ (brs, $\left.2 \mathrm{H}\right), 7.57$ (brs, $3 \mathrm{H}$ ), 5.67 (brs, $5 \mathrm{H}$ ), 3.01 (brs, $2 \mathrm{H}), 1.54$ (brs, $3 \mathrm{H}) .{ }^{19} \mathbf{F}$ NMR $\left(376 \mathrm{MHz}, \mathrm{CD}_{2} \mathrm{Cl}_{2}\right) \delta$-149.7. ${ }^{13} \mathbf{C}$ NMR $\left(100 \mathrm{MHz}, \mathrm{CD}_{2} \mathrm{Cl}_{2}\right) \delta 207.4(\mathrm{CO})$, 132.7 (Ar), $130.8(\mathrm{Ar}), 129.7(\mathrm{Ar}), 122.3(\mathrm{Ar}), 89.8(\mathrm{Cp}), 59.6(\mathrm{C} \equiv \mathrm{C}), 48.7(\mathrm{C} \equiv \mathrm{C}), 21.7\left(\mathrm{CH}_{2}\right), 14.9\left(\mathrm{CH}_{3}\right)$. IR (ATR): $v(\mathrm{CO})=2044,2036 \mathrm{~cm}^{-1}$. HRMS (ESI) calcd. for $\mathrm{C}_{17} \mathrm{H}_{15} \mathrm{O}_{2} \mathrm{Fe}\left[\mathrm{M}-\mathrm{BF}_{4}\right]^{+}:$307.0416, found 307.0415. 


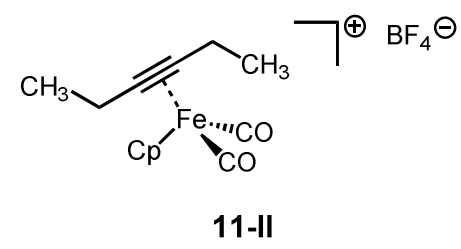

Iron complex 11-II. Prepared following General Procedure A using 3-hexyne (0.7 mL, $6.0 \mathrm{mmol}, 3.0$ equiv), $\mathrm{CpFe}(\mathrm{CO})_{2} \mathrm{I}\left(608 \mathrm{mg}, 2 \mathrm{mmol}, 1.0\right.$ equiv), and $\mathrm{AgBF}_{4}(389 \mathrm{mg}, 2 \mathrm{mmol}, 2.0$ equiv). The reaction mixture was heated at $50{ }^{\circ} \mathrm{C}$ for $2 \mathrm{~h}$, filtered through a pad of Celite, and the filter cake was washed with $\mathrm{CH}_{2} \mathrm{Cl}_{2}(50 \mathrm{~mL})$. The filtrate was evaporated to obtain the desired product as a dark orange solid. The crude solid was recrystallized from $\mathrm{Et}_{2} \mathrm{O} / \mathrm{CH}_{2} \mathrm{Cl}_{2}(1: 1 \mathrm{v} / \mathrm{v})$ to afford 11-II as a pale orange solid. Yield: $534 \mathrm{mg}, 77 \% .{ }^{1} \mathbf{H}$ NMR $\left(400 \mathrm{MHz}, \mathrm{CD}_{2} \mathrm{Cl}_{2}\right) \delta 5.57$ (brs, $\left.5 \mathrm{H}\right), 2.70(\mathrm{~d}, J=6.4$ $\mathrm{Hz}, 4 \mathrm{H}), 1.43(\mathrm{t}, J=6.4 \mathrm{~Hz}, 6 \mathrm{H}) .{ }^{19} \mathbf{F} \mathbf{~ N M R}\left(376 \mathrm{MHz}, \mathrm{CD}_{2} \mathrm{Cl}_{2}\right) \delta-151.9,-152.0 .{ }^{13} \mathbf{C} \mathbf{~ N M R}\left(100 \mathrm{MHz}, \mathrm{CD}_{2} \mathrm{Cl}_{2}\right) \delta 207.8$ (CO), $89.2(\mathrm{Cp}), 50.2(\mathrm{C} \equiv \mathrm{C}), 20.4\left(\mathrm{CH}_{2}\right), 15.1\left(\mathrm{CH}_{3}\right)$. IR (ATR): $v(\mathrm{CO})=2066,2029 \mathrm{~cm}^{-1}$. HRMS (ESI) calcd. for $\mathrm{C}_{13} \mathrm{H}_{15} \mathrm{O}_{2} \mathrm{Fe}\left[\mathrm{M}-\mathrm{BF}_{4}\right]^{+}: 259.0416$, found 259.0414 .

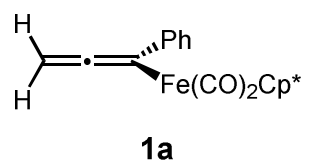

Iron complex 1a. Prepared following General Procedure B using iron complex 1-I ( $91 \mathrm{mg}, 0.2 \mathrm{mmol}, 1.0$ equiv), and $\mathrm{Et}_{3} \mathrm{~N}(31 \mu \mathrm{L}, 0.22 \mathrm{mmol}, 1.1$ equiv) The reaction mixture was stirred at room temperature for $1 \mathrm{~min}$ and the solvent was evaporated to dryness within an additional $2 \mathrm{~min} . \mathrm{Et}_{2} \mathrm{O}(5 \mathrm{~mL})$ was added into the crude solid and the suspension was filtered through a pad of Celite and washed with additional $\mathrm{Et}_{2} \mathrm{O}(10 \mathrm{~mL})$. Solvent was then removed to obtain the desired product 1a as an orange solid. Note: During workup, the temperature should not exceed $40{ }^{\circ} \mathrm{C}$; otherwise, partial isomerization to propargyliron isomer was observed. Yield: $69 \mathrm{mg}, 94 \% .{ }^{1} \mathbf{H}$ NMR $\left(400 \mathrm{MHz}, \mathrm{CD}_{2} \mathrm{Cl}_{2}\right) \delta 7.32-7.30(\mathrm{~m}$, 2H), 7.23-7.19 (m, 2H), 7.08-7.04 (m, 1H), $4.13(\mathrm{~s}, 2 \mathrm{H}), 1.75(\mathrm{~s}, 15 \mathrm{H}) \cdot{ }^{13} \mathbf{C}$ NMR $\left(100 \mathrm{MHz}, \mathrm{CD}_{2} \mathrm{Cl}_{2}\right) \delta 217.5(\mathrm{CO})$, 200.9 (C=$=\mathrm{C}), 145.0$ (Ar), 128.7 (Ar), 127.5 (Ar), 124.6 (Ar), $96.4\left(\underline{C}_{5} \mathrm{Me}_{5}\right), 94.3(\underline{\mathrm{C}}=\mathrm{C}=\mathrm{C}), 59.9(\mathrm{C}=\mathrm{C}=\underline{\mathrm{C}}), 9.4$ $\left(\mathrm{C}_{5} \underline{\mathrm{Me}}_{5}\right)$. IR (ATR, $\mathrm{CCl}_{4}$ solution): $v(\mathrm{CO})=1993,1917 \mathrm{~cm}^{-1}$. HRMS (ESI) calcd. for $\mathrm{C}_{21} \mathrm{H}_{23} \mathrm{O}_{2} \mathrm{Fe}[\mathrm{M}+\mathrm{H}]^{+}: 363.1042$, found 363.1042 .

Alternative procedure for iron complex 1a. To Fp*I ( $100 \mathrm{mg}, 268 \mu \mathrm{mol}, 1.0$ equiv) and $\mathrm{AgBF}_{4}(57.4 \mathrm{mg}, 295 \mu \mathrm{mol}, 1.1$ equiv) was added 1-phenyl-1-propyne $(67 \mu \mathrm{L}, 536 \mu \mathrm{mol}, 2.0$ equiv) then dry toluene $(1.0 \mathrm{~mL})$. The reaction was vigorously stirred until disappearance of red color and even suspension of the grey-orange solid. Hexanes was added $(6 \mathrm{~mL})$ then the contents were flushed through hexanes equilibrated pad of celite then washed with additional hexanes $(3 \times 5 \mathrm{~mL})$ then collected with dry DCM until the filtrate ran colorless $(15 \mathrm{~mL})$. To this solution was added neat triethylamine $(68 \mu \mathrm{L}$, $348 \mu \mathrm{mol}, 1.3$ equiv) causing a color change from orange to cloudy brown. The reaction was stirred for 5 minutes, then concentrated in vacuo to one sixth volume $(2-3 \mathrm{~mL}$ remaining) then hexanes $(20 \mathrm{~mL})$ was added causing the precipitation of a brown solid. The mixture is filtered through a hexanes-equilibrated plug of celite and the yellow-orange filtrated was concentrated to give an orange oil which crystallizes under high vacuum $(75.1 \mathrm{mg}, 77 \%$ over two steps, no detectable propargyliron isomer).

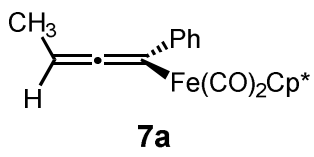

Iron complex 7a Prepared following a literature procedure from iron complex 7-I. ${ }^{1}$ 


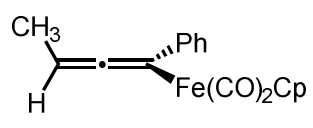

7b

Iron complex 7b. Prepared following General Procedure B using iron complex 7-II ( $93 \mathrm{mg}, 0.2 \mathrm{mmol}, 1.0$ equiv), and $\mathrm{Et}_{3} \mathrm{~N}(31 \mu \mathrm{L}, 0.22 \mathrm{mmol}, 1.1$ equiv). The reaction mixture was stirred at room temperature for $1 \mathrm{~min}$ and the solvent was evaporated within an additional $2 \mathrm{~min} . \mathrm{Et}_{2} \mathrm{O}(5 \mathrm{~mL})$ was added into the crude solid and the suspension was filtered through a pad of Celite and washed with additional $\mathrm{Et}_{2} \mathrm{O}(10 \mathrm{~mL})$. Solvent was then removed to obtain the desired product $7 \mathbf{b}$ as an orange oil. Yield: $59 \mathrm{mg}, 95 \% .{ }^{1} \mathbf{H}$ NMR $\left(400 \mathrm{MHz}, \mathrm{C}_{6} \mathrm{D}_{6}\right) \delta$ 7.46-7.43 (m, 2H), 7.21-7.17 (m, 2H), 7.05-7.01 $(\mathrm{m}, 1 \mathrm{H}), 4.80(\mathrm{q}, J=6.8 \mathrm{~Hz}, 1 \mathrm{H}), 4.11(\mathrm{~s}, 5 \mathrm{H}), 1.71(\mathrm{~d}, J=6.8 \mathrm{~Hz}, 3 \mathrm{H}) \cdot{ }^{13} \mathbf{C} \mathbf{N M R}\left(100 \mathrm{MHz}, \mathrm{C}_{6} \mathrm{D}_{6}\right) \delta 216.1(\mathrm{CO}), 215.8$ (CO), $203.5(\mathrm{C}=\underline{\mathrm{C}}=\mathrm{C}), 147.6(\mathrm{Ar}), 128.3(\mathrm{Ar}), 127.8(\mathrm{Ar}), 124.9(\mathrm{Ar}), 86.1(\underline{\mathrm{C}}=\mathrm{C}=\mathrm{C}), 85.6(\mathrm{Cp}), 73.8(\mathrm{C}=\mathrm{C}=\underline{\mathrm{C}}), 14.6$ $\left(\mathrm{CH}_{3}\right)$. IR (ATR, $\mathrm{CCl}_{4}$ solution): $v(\mathrm{CO})=2010,1955 \mathrm{~cm}^{-1}$. HRMS (ESI) calcd. for $\mathrm{C}_{17} \mathrm{H}_{15} \mathrm{O}_{2} \mathrm{Fe}[\mathrm{M}+\mathrm{H}]^{+}:$307.0416, found 307.0418.

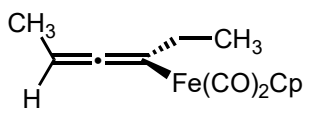

11

Iron complex 11. Prepared following General Procedure B using iron complex 11-II ( $69 \mathrm{mg}, 0.2 \mathrm{mmol}, 1.0$ equiv), and $\mathrm{Et}_{3} \mathrm{~N}$ ( $31 \mu \mathrm{L}, 0.22 \mathrm{mmol}, 1.1$ equiv). The reaction mixture was stirred at room temperature for $1 \mathrm{~min}$ and the solvent was evaporated within an additional $2 \mathrm{~min} . \mathrm{Et}_{2} \mathrm{O}(5 \mathrm{~mL})$ was added into the crude solid and the suspension was filtered through a pad of Celite and washed with additional $\mathrm{Et}_{2} \mathrm{O}(10 \mathrm{~mL})$. Solvent was then removed to obtain the desired product 11 as an orange oil. Yield: $49 \mathrm{mg}, 95 \% .{ }^{1} \mathbf{H}$ NMR $\left(400 \mathrm{MHz}, \mathrm{C}_{6} \mathrm{D}_{6}\right) \delta 4.76-4.70(\mathrm{~m}, 1 \mathrm{H}), 4.12(\mathrm{~s}, 5 \mathrm{H}), 2.20-1.97(\mathrm{~m}$, $2 \mathrm{H}), 1.72(\mathrm{~d}, J=6.8 \mathrm{~Hz}, 3 \mathrm{H}), 1.25(\mathrm{t}, J=7.2 \mathrm{~Hz}, 3 \mathrm{H}) .{ }^{13} \mathbf{C}$ NMR (100 MHz, $\left.\mathrm{C}_{6} \mathrm{D}_{6}\right) \delta 216.4(\mathrm{CO}), 216.3(\mathrm{CO}), 200.0$ $(\mathrm{C}=\underline{\mathrm{C}}=\mathrm{C}), 87.6(\underline{\mathrm{C}}=\mathrm{C}=\mathrm{C}), 85.3(\mathrm{Cp}), 75.9(\mathrm{C}=\mathrm{C}=\underline{\mathrm{C}}), 36.3\left(\mathrm{CH}_{2}\right), 15.4\left(\mathrm{CH}_{3}\right), 15.3\left(\mathrm{CH}_{3}\right)$. IR $\left(\mathrm{ATR}, \mathrm{CCl}_{4}\right.$ solution$)$ : $v(\mathrm{CO})=1954,2010 \mathrm{~cm}^{-1}$. HRMS (ESI) calcd. for $\mathrm{C}_{13} \mathrm{H}_{15} \mathrm{O}_{2} \mathrm{Fe}[\mathrm{M}+\mathrm{H}]^{+}: 259.0416$, found 259.0415.

\section{One pot synthesis of iron complex 3}

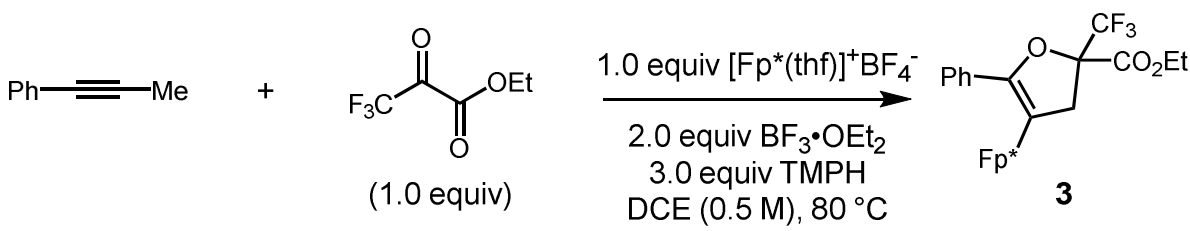

In an argon filled glovebox, a threaded reaction tube $(5 \mathrm{~mL})$ equipped with a magnetic stir bar was charged with $\left[\mathrm{Fp}^{*}(\mathrm{thf})\right]^{+} \mathrm{BF}_{4}^{-}(203 \mathrm{mg}, 0.50 \mathrm{mmol}, 1.0$ equiv), 1-phenyl-1-propyne $(125 \mu \mathrm{L}, 1.0 \mathrm{mmol}, 2.0$ equiv), ethyl trifluoropyruvate $\left(66 \mu \mathrm{L}, 0.50 \mathrm{mmol}, 1.0\right.$ equiv), and $\mathrm{DCE}(1 \mathrm{~mL})$. Subsequently, $\mathrm{BF}_{3} \cdot \mathrm{OEt}_{2}(123 \mu \mathrm{L}, 1.0 \mathrm{mmol}, 2.0$ equiv $)$ and distilled TMPH ( $235 \mu \mathrm{L}, 1.5 \mathrm{mmol}, 3.0$ equiv) were added in rapid succession and the reaction tube was fitted with a septum cap and removed from the glovebox. The reaction mixture was stirred for $12 \mathrm{~h}$ at $80{ }^{\circ} \mathrm{C}$, and the crude material was loaded directly onto a silica gel column packed with hexanes and purified by chromatography (hexanes/EtOAc $=9: 1$ to $4: 1$ ) to give the product 3 as an oily yellow solid which crystallized upon storage under nitrogen. Yield: $135 \mathrm{mg}, 51 \%$. ${ }^{1} \mathbf{H}$ NMR $\left(500 \mathrm{MHz}, \mathrm{CD}_{2} \mathrm{Cl}_{2}\right) \delta 7.61(\mathrm{~d}, J=7.0 \mathrm{~Hz}, 2 \mathrm{H}), 7.35(\mathrm{t}, J=7.0 \mathrm{~Hz}, 2 \mathrm{H}), 7.30-7.27(\mathrm{~m}, 1 \mathrm{H}), 4.31(\mathrm{q}, J=7.0 \mathrm{~Hz}, 2 \mathrm{H}), 3.25-$ $3.15(\mathrm{~m}, 2 \mathrm{H}), 1.70(\mathrm{~s}, 15 \mathrm{H}), 1.34(\mathrm{t}, J=7.0 \mathrm{~Hz}, 3 \mathrm{H}) .{ }^{19} \mathbf{F} \mathbf{N M R}(470 \mathrm{~Hz})-75.9 .{ }^{13} \mathbf{C} \mathbf{N M R}\left(125 \mathrm{MHz}, \mathrm{CD}_{2} \mathrm{Cl}_{2}\right) \delta 217.0$

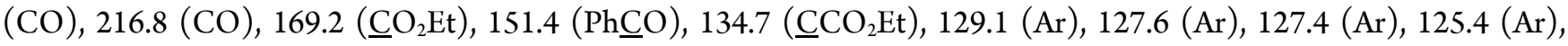
$124.3\left(\mathrm{q}, J=281 \mathrm{~Hz}, \mathrm{CF}_{3}\right), 108.0\left(\mathrm{Fp}^{*} \underline{\mathrm{C}}\right), 96.5\left(\underline{\mathrm{C}}_{5} \mathrm{Me}_{5}\right), 83.4(\mathrm{q}, J=23 \mathrm{~Hz}, \underline{\mathrm{CCF}} 3), 62.2\left(\underline{\mathrm{CH}}_{2} \mathrm{CH}_{3}\right), 52.7\left(\mathrm{CH}_{2}\right), 13.8$ $\left(\mathrm{CH}_{3}\right), 9.4\left(\mathrm{C}_{5} \underline{\mathrm{Me}}_{5}\right)$. IR (ATR): $v(\mathrm{CO})=1993,1931,1753 \mathrm{~cm}^{-1}$. HRMS (ESI) calcd. for $\mathrm{C}_{26} \mathrm{H}_{28} \mathrm{~F}_{3} \mathrm{FeO}_{5}[\mathrm{M}+\mathrm{H}]^{+}$: 533.1233, found: 533.1232 . 


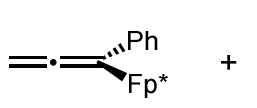

$1 a$<smiles>CCOC(=O)C(=O)C(F)(F)F</smiles>

(1.5 equiv)
$\overrightarrow{D C M}(0.25 \mathrm{M}), 24{ }^{\circ} \mathrm{C}$

$\operatorname{DCM}(0.25 \mathrm{M}), 24^{\circ} \mathrm{C}$

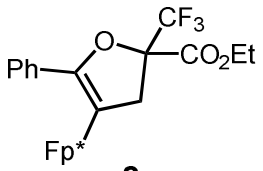

3

In an argon filled glovebox, a threaded reaction tube $(5 \mathrm{~mL})$ equipped with a magnetic stir bar was charged with ethyl trifluoropyruvate ( $99 \mu \mathrm{L}, 0.75 \mathrm{mmol}, 1.5$ equiv). A solution of freshly-prepared allenyliron complex $1 \mathrm{a}(182 \mathrm{mg}, 0.50 \mathrm{mmol}$, 1.0 equiv) in $\mathrm{CH}_{2} \mathrm{Cl}_{2}(0.5 \mathrm{~mL})$ was then added, and the reaction tube was fitted with a septum cap and removed from the glovebox. The reaction mixture was then stirred at room temperature $\left(24^{\circ} \mathrm{C}\right)$ for $2.5 \mathrm{~h}$. The crude mixture was concentrated in vacuo and purified by flash column chromatography on neutral aluminum oxide (hexanes $/ \mathrm{CH}_{2} \mathrm{Cl}_{2}=1: 0$ to $10: 1$ to $0: 1$ ) and subsequent recrystallization to provide the desired product 3 as a bright yellow solid. A crystal suitable for Xray diffraction was obtained by carefully laying hexanes onto a dichloromethane solution of the corresponding compound. Yield: $128 \mathrm{mg}$, 48\%. EA calcd. for $\mathrm{C}_{26} \mathrm{H}_{27} \mathrm{~F}_{3} \mathrm{FeO}_{5}$ : C 58.66, H 5.11, found: C 58.74, $\mathrm{H}$ 5.14.

\section{One pot synthesis of iron complex 6}

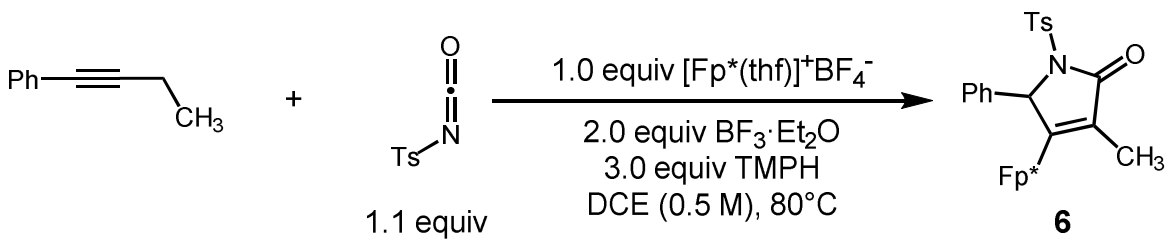

To a septum capped, argon filled reaction tube $(5 \mathrm{~mL})$ equipped with a magnetic stir bar and charged with $\left[\mathrm{Fp}^{*}(\mathrm{thf})\right]^{+} \mathrm{BF}_{4}$ ( $203 \mathrm{mg}, 0.50 \mathrm{mmol}, 1.0$ equiv) was added 1-phenyl-1-propyne $(125 \mu \mathrm{L}, 1.0 \mathrm{mmol}, 2.0$ equiv), tosyl isocyanate $(82 \mu \mathrm{L}$, $0.54 \mathrm{mmol}, 1.1$ equiv) and DCE $(1 \mathrm{~mL})$. Then, in rapid succession, $\mathrm{BF}_{3} \cdot \mathrm{OEt}_{2}(123 \mu \mathrm{L}, 1.0 \mathrm{mmol}, 2.0$ equiv $)$ and distilled TMPH $\left(235 \mu \mathrm{L}, 1.5 \mathrm{mmol}, 3.0\right.$ equiv) were added. After stirring for $12 \mathrm{~h}$ at $80^{\circ} \mathrm{C}$, the crude reaction mixture was purified directly by chromatography $\left(\mathrm{CH}_{2} \mathrm{Cl}_{2} / \mathrm{EtOAc}=1: 0\right.$ to $\left.49: 1\right)$ to give the product 6 as an oily yellow solid which crystallized upon storage under nitrogen. A crystal suitable for X-ray diffraction was obtained by slowly cooling a solution in hot $\mathrm{CH}_{2} \mathrm{Cl}_{2} /$ hexanes $(1: 20 \mathrm{v} / \mathrm{v})$. Yield: $126 \mathrm{mg}, 44 \% .{ }^{1} \mathbf{H}$ NMR $\left(400 \mathrm{MHz}, \mathrm{CD}_{2} \mathrm{Cl}_{2}\right) \delta 7.57$ (brs, $\left.1 \mathrm{H}\right), 7.41-7.40(\mathrm{~m}, 1 \mathrm{H})$, $7.27(\mathrm{t}, J=7.2 \mathrm{~Hz}, 1 \mathrm{H}), 7.21-7.19(\mathrm{~m}, 2 \mathrm{H}), 7.04-7.02(\mathrm{~m}, 2 \mathrm{H}), 6.98(\mathrm{brs}, 1 \mathrm{H}), 6.40(\mathrm{brs}, 1 \mathrm{H}), 5.37(\mathrm{~s}, 1 \mathrm{H}), 2.32(\mathrm{~s}, 3 \mathrm{H})$, $2.00(\mathrm{~s}, 3 \mathrm{H}), 1.73(\mathrm{~s}, 15 \mathrm{H}) .{ }^{13} \mathrm{C}$ NMR $\left(100 \mathrm{MHz}, \mathrm{CD}_{2} \mathrm{Cl}_{2}\right) \delta 216.1(\mathrm{CO}), 213.7(\mathrm{CO}), 167.4(\underline{\mathrm{CON}}), 143.7(\mathrm{Ar}), 139.3$ (Ar), 137.9 (Ar), 136.8 (Ar), 129.0 (Ar), 128.7 (Ar), 128.22 (Ar), $128.16(\mathrm{Ar}), 127.4(\underline{\mathrm{C}}=\mathrm{C}), 125.2(\mathrm{C}=\underline{\mathrm{C}}), 96.8\left(\underline{\mathrm{C}}_{5} \mathrm{Me}_{5}\right)$, $75.8(\mathrm{Ph} \underline{\mathrm{CHN}}), 21.2\left(\mathrm{CH}_{3}\right), 14.0\left(\mathrm{CH}_{3}\right), 9.5\left(\mathrm{C}_{5} \underline{\mathrm{Me}}_{5}\right)$. IR (ATR): $v(\mathrm{CO})=1993,1940,1730 \mathrm{~cm}^{-1}$. HRMS (ESI) calcd. for $\mathrm{C}_{30} \mathrm{H}_{32} \mathrm{FeNO}_{5} \mathrm{~S}[\mathrm{M}+\mathrm{H}]^{+}:$: 574.1345, found: 574.1340 .

\section{Synthesis of iron complex 6 starting from $\eta^{1}$-allenyl pentamethylcyclopentadienyliron dicarbonyl complex 7a}
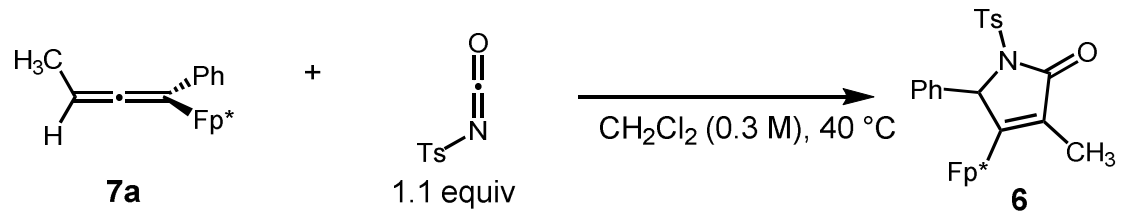

In an argon filled glovebox, a threaded reaction tube $(5 \mathrm{~mL})$ equipped with a magnetic stir bar was charged with TsNCO (37 $\mu \mathrm{L}, 0.22 \mathrm{mmol}, 1.1$ equiv). A solution of freshly-prepared allenyliron complex $7 \mathbf{a}(75 \mathrm{mg}, 0.20 \mathrm{mmol}, 1.0$ equiv) in $\mathrm{CH}_{2} \mathrm{Cl}_{2}(0.6 \mathrm{~mL})$ was then added. The reaction tube was fitted with a septum cap and removed from the glovebox. The reaction mixture was then stirred at $40^{\circ} \mathrm{C}$ for $16 \mathrm{~h}$. Subsequently, the reaction mixture was concentrated in vacuo, and the crude material was purified by flash column chromatography on neutral aluminum oxide (hexanes $/ \mathrm{CH}_{2} \mathrm{Cl}_{2}=1: 0$ to $10: 1$ to $0: 1)$ and subsequent recrystallization from hexanes $/ \mathrm{CH}_{2} \mathrm{Cl}_{2}(10: 1 \mathrm{v} / \mathrm{v})$ to provide the desired product 6 as a bright yellow solid. Yield: $86 \mathrm{mg}, 75 \%$. EA calcd. for $\mathrm{C}_{30} \mathrm{H}_{31} \mathrm{FeNO}_{5} \mathrm{~S}$ : C 62.83, H 5.45, found: C 62.57, H 5.41. 


\section{Synthesis of iron complex $6^{\prime}$ starting from $\eta^{1}$-allenyl pentamethylcyclopentadienyliron dicarbonyl complex 7 a}

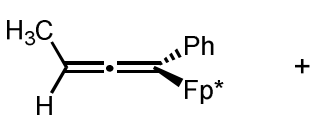

$7 a$

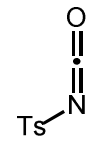

1.1 equiv

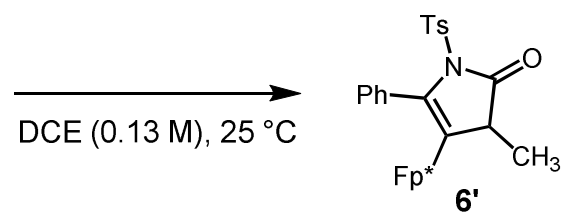

In an argon filled glovebox, a threaded reaction tube $(5 \mathrm{~mL})$ equipped with a magnetic stir bar was charged with a solution of $7 \mathbf{a}(98 \mathrm{mg}, 0.26 \mathrm{mmol}, 1.0$ equiv) in dry DCE $(2 \mathrm{~mL})$. Tosyl isocyanate $(45 \mu \mathrm{L}, 0.29 \mathrm{mmol}, 1.1$ equiv) was added. The reaction tube was then fitted with a septum cap and removed from the glovebox, whereupon the reaction was stirred at room temperature for $1 \mathrm{~h}$. The reaction mixture was loaded directly onto a column equilibrated with hexanes $/ \mathrm{CH}_{2} \mathrm{Cl}_{2}$ $(1: 1 \mathrm{v} / \mathrm{v})$ and quickly purified by chromatography (hexanes $/ \mathrm{CH}_{2} \mathrm{Cl}_{2} / \mathrm{EtOAc}=9: 1: 0$ to 1:1:0 to 0:49:1) to afford a bright yellow amorphous solid $\mathbf{6}^{\mathbf{}}$ that dried to a foam under high vacuum. Yield: $64 \mathrm{mg}, 43 \% .{ }^{1} \mathbf{H} \mathbf{~ N M R}\left(400 \mathrm{MHz}, \mathrm{CDCl}_{3}\right) \delta$ 7.61-7.59 (d, $J=8.3 \mathrm{~Hz}, 2 \mathrm{H}), 7.354-7.348(\mathrm{~m}, 3 \mathrm{H}), 7.29-7.27(\mathrm{~m}, 4 \mathrm{H}), 2.74(\mathrm{q}, J=7.6 \mathrm{~Hz}, 1 \mathrm{H}), 2.42(\mathrm{~s}, 3 \mathrm{H}), 1.62(\mathrm{~s}$, $15 \mathrm{H}), 1.21(\mathrm{~d}, J=7.6 \mathrm{~Hz}, 3 \mathrm{H}) .{ }^{13} \mathbf{C} \mathbf{~ N M R}(100 \mathrm{~Hz}): 217.3(\mathrm{CO}), 215.8(\mathrm{CO}), 183.2(\mathrm{CON}), 144.3(\mathrm{NC}=\mathrm{C}), 138.1(\mathrm{Ar})$, 137.3 (Ar), 137.0 (Ar), 136.6 (Ar), 129.7 (Ar), $129.2(\mathrm{Ar}), 127.7$ (Ar), 127.6 (Ar), 126.3 (C=ㅡ) $, 96.4\left(\underline{\mathrm{C}}_{5} \mathrm{Me}_{5}\right), 21.4$ $\left(\mathrm{CH}_{3}\right), 18.0\left(\mathrm{CH}_{3}\right), 9.3\left(\mathrm{C}_{5} \underline{\mathrm{Me}}_{5}\right)$ (the signal for $\mathrm{CCH}_{3}$ was not observed and is likely obscured by the solvent signal). LRMS (ESI) calcd. for $\mathrm{C}_{30} \mathrm{H}_{32} \mathrm{FeNO}_{5} \mathrm{~S}[\mathrm{M}+\mathrm{H}]^{+}$: 574.14, found: 573.95 .

\subsection{Demetallation reactions of iron complex}

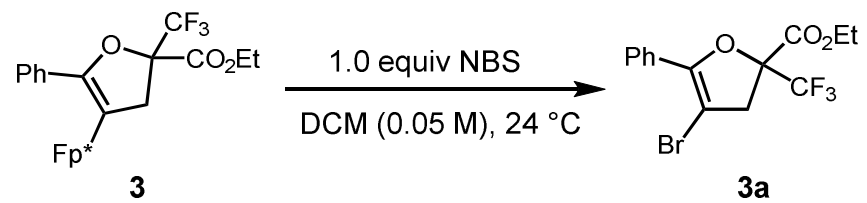

Ethyl 4-bromo-5-phenyl-2-(trifluoromethyl)-2,3-dihydrofuran-2-carboxylate (3a). To a solution of recentlychromatographed iron complex 3 (13 mg, $23 \mu \mathrm{mol}, 1.0$ equiv) in dry $\mathrm{CH}_{2} \mathrm{Cl}_{2}(0.5 \mathrm{~mL})$ was added NBS $(4.2 \mathrm{mg}, 23 \mu \mathrm{mol}$, 1.0 equiv). The mixture was stirred at $24^{\circ} \mathrm{C}$ under air and monitored by thin layer chromatography until disappearance of the starting material $(1 \mathrm{~h})$, concentrated in vacuo, and the crude residue was purified by preparatory thin layer chromatography $\left(\mathrm{SiO}_{2}\right.$, hexanes/EtOAc $\left.=85: 15\right)$ to give 3a. Yield: $4.6 \mathrm{mg}$, 54\%. Alternatively, 3a could be obtained using stoichiometric $\mathrm{Br}_{2}$ in place of NBS, though side product formation was apparent and a reduced yield of desired product was observed. Yield: $1.3 \mathrm{mg}, 15 \% .{ }^{1} \mathbf{H}$ NMR $\left(400 \mathrm{MHz}, \mathrm{CDCl}_{3}\right) \delta$ 7.92-7.90 (m, 2H), 7.42-7.40 (m, 3H), $4.36(\mathrm{q}, J=7.2 \mathrm{~Hz}$, $2 \mathrm{H}), 3.54(\mathrm{~s}, 2 \mathrm{H}), 1.35(\mathrm{t}, J=7.2 \mathrm{~Hz}, 3 \mathrm{H}) .{ }^{19} \mathbf{F} \mathbf{~ N M R}\left(376 \mathrm{MHz}, \mathrm{CDCl}_{3}\right) \delta$-78.9. ${ }^{13} \mathbf{C} \mathbf{N M R}\left(100 \mathrm{MHz}, \mathrm{CDCl}_{3}\right) \delta 166.1$ $\left(\underline{\mathrm{CO}}{ }_{2} \mathrm{Et}\right), 149.7(\mathrm{PhCO}), 129.7(\mathrm{Ar}), 128.3(\mathrm{Ar}), 128.1(\mathrm{Ar}), 127.3(\mathrm{Ar}), 122.7$ (q, J = 282 Hz, CF3), $86.5(\mathrm{CBr}), 83.5$ (q, $\left.J=31 \mathrm{~Hz}, \underline{\mathrm{CCF}_{3}}\right), 63.3\left(\mathrm{CO}_{2} \underline{\mathrm{CH}_{2}} \mathrm{CH}_{3}\right), 43.4\left(\mathrm{CH}_{2}\right), 13.9\left(\mathrm{CO}_{2} \mathrm{CH}_{2} \underline{\mathrm{C}} \mathrm{H}_{3}\right)$. HRMS (ESI) calcd. for $\mathrm{C}_{14} \mathrm{H}_{13} \mathrm{O}_{3} \mathrm{BrF}_{3}$ $[\mathrm{M}+\mathrm{H}]^{+}$: 364.9995, found: 364.9998 .

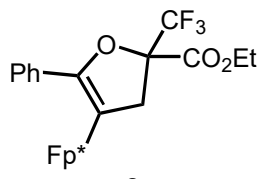

3

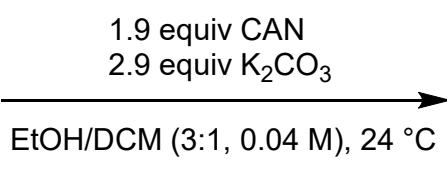

EtOH/DCM $(3: 1,0.04 \mathrm{M}), 24^{\circ} \mathrm{C}$

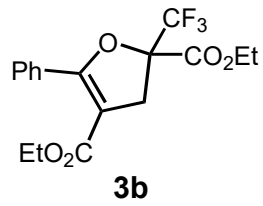

$3 b$

Diethyl 5-phenyl-2-(trifluoromethyl)-2,3-dihydrofuran-2,4-dicarboxylate (3b). To a solution of recentlychromatographed $3\left(29.2 \mathrm{mg}, 54.9 \mu \mathrm{mol}, 1.0\right.$ equiv) in dry $\mathrm{CH}_{2} \mathrm{Cl}_{2}(0.3 \mathrm{~mL})$ was added solid $\mathrm{K}_{2} \mathrm{CO}_{3}(22.2 \mathrm{mg}, 161 \mu \mathrm{mol}$, 2.9 equiv), $\mathrm{EtOH}(1.0 \mathrm{~mL})$, and ceric ammonium nitrate (CAN) $(57.4 \mathrm{mg}, 105 \mu \mathrm{mol}, 1.9$ equiv). The mixture was stirred in air at $24^{\circ} \mathrm{C}$ until disappearance of the starting material $(2 \mathrm{~h})$. The mixture was then filtered through a plug of silica, con- 
centrated in vacuo and purified by preparatory plate chromatography $\left(\mathrm{SiO}_{2}\right.$, hexanes/EtOAc $\left.=85: 15\right)$ to give 3b. Yield: $8.5 \mathrm{mg}, 43 \% .{ }^{1} \mathbf{H}$ NMR $\left(600 \mathrm{MHz}, \mathrm{CDCl}_{3}\right) \delta$ 7.86-7.85 (m, 2H), 7.47-7.45 (m, 1H), 7.43-7.40 (m, 2H), $4.37(\mathrm{q}, J=7.2$ $\mathrm{Hz}, 2 \mathrm{H}), 4.18-4.15(\mathrm{~m}, 2 \mathrm{H}), 3.60-3.53(\mathrm{~m}, 2 \mathrm{H}), 1.36(\mathrm{t}, J=7.2 \mathrm{~Hz}, 3 \mathrm{H}), 1.24(\mathrm{t}, J=7.2 \mathrm{~Hz}, 3 \mathrm{H}) .{ }^{19} \mathbf{F}$ NMR $(282 \mathrm{MHz}$, $\left.\mathrm{CDCl}_{3}\right) \delta$-78.6. ${ }^{13} \mathrm{C}$ NMR $\left(151 \mathrm{MHz}, \mathrm{CDCl}_{3}\right) \delta 165.9\left(\mathrm{CO}_{2} \mathrm{Et}\right), 163.5\left(\mathrm{CO}_{2} \mathrm{Et}\right), 163.3(\mathrm{PhCO}), 131.1(\mathrm{Ar}), 129.5(\mathrm{Ar})$, $\left.128.1(\mathrm{Ar}), 127.8(\mathrm{Ar}), 122.7\left(\mathrm{q}, J=283 \mathrm{~Hz}, \mathrm{CF}_{3}\right), 101.9(\underline{\mathrm{CCO}} \mathrm{Et}), 84.6(\mathrm{q}, J=31 \mathrm{~Hz}, \underline{\mathrm{C} C F})_{3}\right), 63.3\left(\mathrm{CO}_{2} \underline{\mathrm{C}} \mathrm{H}_{2} \mathrm{CH}_{3}\right), 60.5$ $\left.\left(\mathrm{CO}_{2} \underline{\mathrm{C}} \mathrm{H}_{2} \mathrm{CH}_{3}\right), 37.4\left(\mathrm{CH}_{2}\right), 14.2\left(\mathrm{CO}_{2} \mathrm{CH}_{2} \underline{\mathrm{CH}}\right)_{3}\right), 14.0\left(\mathrm{CO}_{2} \mathrm{CH}_{2} \underline{\mathrm{C}} \mathrm{H}_{3}\right)$.
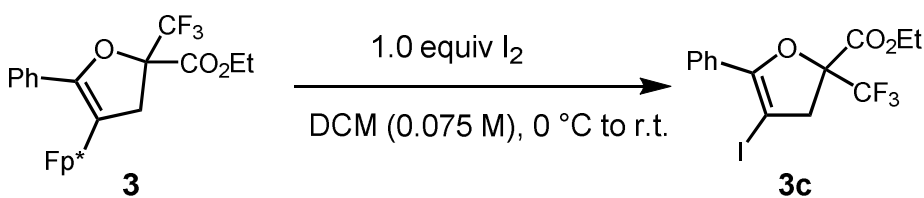

Ethyl 4-iodo-5-phenyl-2-(trifluoromethyl)-2,3-dihydrofuran-2-carboxylate (3c). To a solution of recentlychromatographed iron complex 3 (77.8 mg, $0.15 \mathrm{mmol}, 1.0$ equiv) in dry $\mathrm{CH}_{2} \mathrm{Cl}_{2}(0.5 \mathrm{~mL})$ was added $\mathrm{I}_{2}(0.1 \mathrm{M}$ in $\mathrm{CH}_{2} \mathrm{Cl}_{2}, 1.5 \mathrm{~mL}, 1.0$ equiv) at $0{ }^{\circ} \mathrm{C}$. The reaction mixture was stirred at $24^{\circ} \mathrm{C}$ for $2 \mathrm{~h}$, concentrated in vacuo, and the crude residue was purified by flash column chromatography (hexanes/EtOAc $=85: 15$ ) to give 3c. Yield: $25 \mathrm{mg}$, $42 \%$. Additionally, Fp*I was recovered (34 mg, 63\%). ${ }^{1} \mathbf{H}$ NMR $\left(400 \mathrm{MHz}, \mathrm{CDCl}_{3}\right) \delta 7.62-7.59(\mathrm{~m}, 2 \mathrm{H}), 7.37-7.35(\mathrm{~m}, 3 \mathrm{H}), 4.34(\mathrm{q}, J=$ $7.2 \mathrm{~Hz}, 2 \mathrm{H}), 3.31(\mathrm{~d}, J=2.4 \mathrm{~Hz}, 2 \mathrm{H}), 1.33(\mathrm{t}, J=7.2 \mathrm{~Hz}, 3 \mathrm{H}) .{ }^{19} \mathbf{F} \mathbf{N M R}\left(376 \mathrm{MHz}, \mathrm{CDCl}_{3}\right)-78.8 .{ }^{13} \mathbf{C} \mathbf{~ N M R}(100 \mathrm{MHz}$, $\left.\mathrm{CDCl}_{3}\right) \delta 166.2\left(\underline{\mathrm{CO}}_{2} \mathrm{Et}\right), 153.5(\mathrm{Ph} \underline{\mathrm{CO}}), 129.9(\mathrm{Ar}), 128.8(\mathrm{Ar}), 128.2(\mathrm{Ar}), 127.9(\mathrm{Ar}), 122.8\left(\mathrm{q}, J=282 \mathrm{~Hz}, \mathrm{CF}_{3}\right), 84.8$ $\left.(\mathrm{q}, J=31 \mathrm{~Hz}, \underline{\mathrm{C} C F})_{3}\right), 63.2\left(\mathrm{CO}_{2} \underline{\mathrm{C}} \mathrm{H}_{2} \mathrm{CH}_{3}\right), 51.8(\mathrm{CI}), 47.1\left(\mathrm{CH}_{2}\right), 14.0\left(\mathrm{CO}_{2} \mathrm{CH}_{2} \underline{\mathrm{C}}{ }_{3}\right)$. LRMS (ESI) calcd. for $\mathrm{C}_{14} \mathrm{H}_{13} \mathrm{O}_{3} \mathrm{IF}_{3}[\mathrm{M}+\mathrm{H}]^{+}$: 412.99, found: 412.85 .
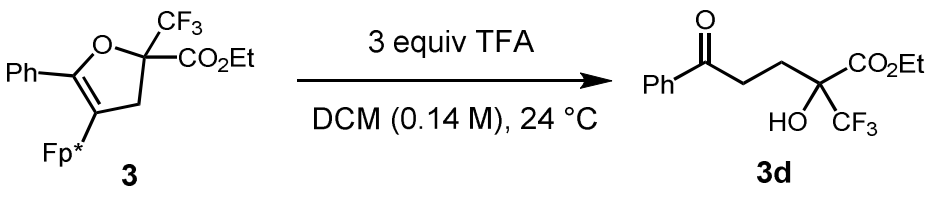

Ethyl 2-hydroxy-5-oxo-5-phenyl-2-(trifluoromethyl)pentanoate (3d). To a solution of recently-chromatographed iron complex 3 (37 mg, $69 \mu \mathrm{mol}, 1.0$ equiv) in $\mathrm{CH}_{2} \mathrm{Cl}_{2}(0.5 \mathrm{~mL}$, stored in air on the benchtop) was added trifluoroacetic acid $(10.7 \mu \mathrm{L}, 0.14 \mathrm{mmol}, 2.0$ equiv). The reaction mixture was stirred overnight $(12 \mathrm{~h})$ under air. The purplish mixture was filtered through a short plug of basic aluminum oxide, washed with $\mathrm{CH}_{2} \mathrm{Cl}_{2}$, and the filtrate concentrated in vacuo to give the crude residue, which was purified by preparatory plate chromatography $\left(\mathrm{SiO}_{2}\right.$, hexanes $\left./ \mathrm{EtOAc}=85: 15\right)$ to afford 3d. Yield: $21 \mathrm{mg}, 98 \% .{ }^{1} \mathbf{H}$ NMR $\left(400 \mathrm{MHz}, \mathrm{CDCl}_{3}\right) \delta 7.95(\mathrm{~d}, J=7.4 \mathrm{~Hz}, 2 \mathrm{H}), 7.58(\mathrm{t}, J=7.4 \mathrm{~Hz}, 1 \mathrm{H}), 7.47(\mathrm{t}, J=7.6$ $\mathrm{Hz}, 2 \mathrm{H}), 4.46-4.31(\mathrm{~m}, 2 \mathrm{H}), 4.00(\mathrm{~s}, 1 \mathrm{H}), 3.28-3.19(\mathrm{~m}, 1 \mathrm{H}), 2.95-2.87(\mathrm{~m}, 1 \mathrm{H}), 2.53-2.46(\mathrm{~m}, 1 \mathrm{H}), 2.43-2.36(\mathrm{~m}, 1 \mathrm{H})$, $1.35(\mathrm{t}, J=7.1 \mathrm{~Hz}, 3 \mathrm{H}) .{ }^{19} \mathbf{F}$ NMR $\left(376 \mathrm{MHz}, \mathrm{CDCl}_{3}\right) \delta-78.3 .{ }^{13} \mathbf{C} \mathbf{N M R}\left(100 \mathrm{MHz}, \mathrm{CDCl}_{3}\right) \delta 198.2(\mathrm{PhCO}), 169.8$ ( $\left.\underline{\mathrm{CO}}_{2} \mathrm{Et}\right), 136.4(\mathrm{Ar}), 133.4(\mathrm{Ar}), 128.7(\mathrm{Ar}), 128.1(\mathrm{Ar}), 123.5\left(\mathrm{q}, J=285 \mathrm{~Hz}, \mathrm{CF}_{3}\right), 64.1\left(\mathrm{CO}_{2} \underline{\mathrm{CH}}_{2} \mathrm{CH}_{3}\right), 31.9$ $\left.\left(\mathrm{CO}_{\mathrm{CH}} \mathrm{CH}_{2}\right), 26.2\left(\mathrm{COCH}_{2} \underline{\mathrm{CH}}\right)_{2}\right), 13.9\left(\mathrm{CO}_{2} \mathrm{CH}_{2} \underline{\mathrm{CH}} 3\right)$ (the quaternary carbon was not observed). LRMS (ESI) calcd. for $\mathrm{C}_{14} \mathrm{H}_{14} \mathrm{~F}_{3} \mathrm{O}_{3}\left[\mathrm{M}-\mathrm{H}_{2} \mathrm{O}\right]^{+}:$287.09, found: 286.95 .

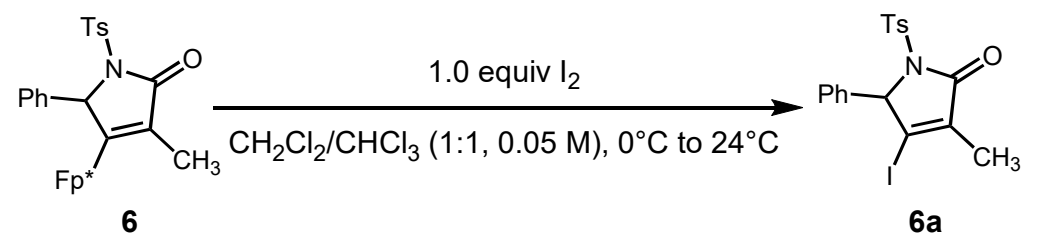

4-Iodo-3-methyl-5-phenyl-1-tosyl-1,5-dihydro-2H-pyrrol-2-one (6a). To a solution of recrystallized iron complex 6

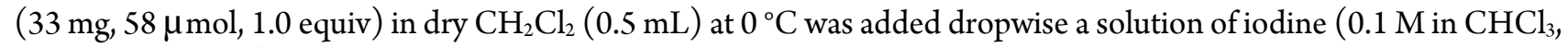
$460 \mu \mathrm{L}, 0.80$ equiv) over 15 minutes. The mixture was stirred at $0{ }^{\circ} \mathrm{C}$ for 30 minutes and then at room temperature for another $2 \mathrm{~h}$. The solution was chromatographed directly (hexanes/EtOAc $=95: 5$ to the desired product and 85:15 to 4:1 to 
Fp*I) to afford 6a. Yield: $12 \mathrm{mg}, 59 \%$. Additionally, Fp*I was recovered (9.2 mg, 53\%). Yield of 6a was much lower (7.2 $\mathrm{mg}, 31 \%)$ when 1.0 equiv each of $\mathbf{6 a}$ and $\mathrm{I}_{2}$ were used instead. ${ }^{1} \mathbf{H} \mathbf{N M R}\left(400 \mathrm{MHz}, \mathrm{CDCl}_{3}\right) \delta 7.39-7.37(\mathrm{~m}, 3 \mathrm{H}), 7.32$ $7.28(\mathrm{~m}, 2 \mathrm{H}), 7.10(\mathrm{~d}, J=8.0 \mathrm{~Hz}, 2 \mathrm{H}), 7.05(\mathrm{~d}, J=8.0 \mathrm{~Hz}, 2 \mathrm{H}), 5.53(\mathrm{~s}, 1 \mathrm{H}), 2.36(\mathrm{~s}, 3 \mathrm{H}), 1.93(\mathrm{~s}, 3 \mathrm{H}) .{ }^{13} \mathbf{C} \mathbf{~ N M R}(400$ $\mathrm{MHz}_{\mathrm{CDCl}}$ ) $\delta 165.8(\underline{\mathrm{CON}}), 144.8(\mathrm{Ar}), 139.6(\mathrm{Ar}), 135.5(\mathrm{Ar}), 134.0(\mathrm{Ar}), 129.3(\mathrm{Ar}), 129.2(\mathrm{Ar}), 128.8(\mathrm{Ar}), 128.4$ $(\mathrm{Ar}), 128.0\left(\mathrm{IC}=\mathrm{CCH}_{3}\right), 118.9\left(\mathrm{IC}=\mathrm{CCH}_{3}\right), 71.5(\mathrm{Ph} \underline{\mathrm{CHN}}), 21.7\left(\mathrm{CH}_{3}\right), 13.4\left(\mathrm{CH}_{3}\right)$. LRMS (ESI) calcd. for $\mathrm{C}_{18} \mathrm{H}_{17} \mathrm{INO}_{3} \mathrm{~S}[\mathrm{M}+\mathrm{H}]^{+}: 454.00$, found: 453.85 .

\subsection{Synthesis of $\mathrm{N}$-arylpyrazoles}

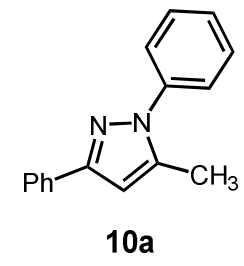

5-Methyl-1,3-diphenyl-1H-pyrazole (10a). Prepared following General Procedure $\mathbf{C}$ using iron complex $7 \mathbf{b}$ (59 mg, $0.19 \mathrm{mmol}, 1.0$ equiv), and phenyldiazonium tetrafluoroborate $(56 \mathrm{mg}, 0.29 \mathrm{mmol}, 1.5$ equiv). The reaction mixture was stirred at $60{ }^{\circ} \mathrm{C}$ for $12 \mathrm{~h}$ and the crude residue was purified by flash column chromatography (hexanes $/$ EtOAc $=30: 1$ to 10:1). The desired product 10a was obtained as a white solid. Regioisomeric ratio ( $>100: 1 \mathrm{r} . \mathrm{r})$ was determined by ${ }^{1} \mathrm{H}$ NMR of the crude residue. Yield: $12 \mathrm{mg}, 27 \% .{ }^{1} \mathbf{H}$ NMR $\left(400 \mathrm{MHz}, \mathrm{CDCl}_{3}\right) \delta$ 7.87-7.85 (m, 2H), 7.54-7.47 (m, 4H), 7.42-7.37 (m, 3H), 7.33-7.30 (m, 1H), $6.53(\mathrm{~s}, 1 \mathrm{H}), 2.39(\mathrm{~s}, 3 \mathrm{H}) .{ }^{13} \mathbf{C}$ NMR (100 MHz, CDCl $) \delta 151.5(\mathrm{PhCN}), 140.2$ $\left(\mathrm{CH}_{3} \underline{\mathrm{CN}}\right), 140.0$ (Ar), 133.4 (Ar), 129.1 (Ar), 128.6 (Ar), 127.8 (Ar), 127.6 (Ar), 125.7(Ar), $125.0(\mathrm{Ar}), 104.4(\mathrm{CH})$, $12.6\left(\mathrm{CH}_{3}\right)$. The spectra are in accordance with the literature data. ${ }^{2}$

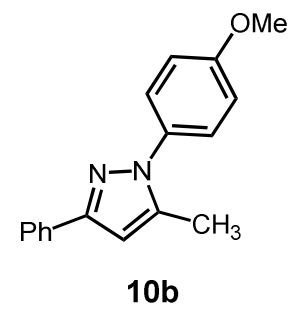

1-(4-Methoxyphenyl)-5-methyl-3-phenyl-1H-pyrazole (10b). Prepared following General Procedure $\mathbf{C}$ using iron complex $7 \mathbf{b}$ ( $53 \mathrm{mg}, 0.17 \mathrm{mmol}, 1.0$ equiv), and 4-methoxybenzenediazonium tetrafluoroborate (56 $\mathrm{mg}, 0.25 \mathrm{mmol}, 1.5$ equiv). The reaction mixture was stirred at $60^{\circ} \mathrm{C}$ for $12 \mathrm{~h}$ and the crude residue was purified by flash column chromatography (hexanes $/$ EtOAc $=30: 1$ to 5:1). The desired product $\mathbf{1 0 b}$ was obtained as a pale yellow solid. Regioisomeric ratio (>100:1 r.r.) was determined by ${ }^{1} \mathrm{H}$ NMR of the crude residue. Yield: $16 \mathrm{mg}, 36 \% .{ }^{1} \mathbf{H} \mathbf{N M R}\left(500 \mathrm{MHz}, \mathrm{CDCl}_{3}\right) \delta 7.86-$ 7.84 (m, 2H), 7.43-7.38 (m, 4H), 7.31-7.28 (m, 1H), 7.00-6.99 (m, 2H), $6.50(\mathrm{~s}, 1 \mathrm{H}), 3.86(\mathrm{~s}, 3 \mathrm{H}), 2.33(\mathrm{~s}, 3 \mathrm{H}) .{ }^{13} \mathbf{C}$ NMR $\left(125 \mathrm{MHz}, \mathrm{CDCl}_{3}\right) \delta 159.1$ ( $\left.\underline{\mathrm{COMe}}\right), 151.1(\mathrm{Ph} \underline{\mathrm{CN}}), 140.3\left(\underline{\mathrm{CH}}_{3} \mathrm{CN}\right), 133.5(\mathrm{Ar}), 133.1$ (Ar), $128.5(\mathrm{Ar}), 127.6$ (Ar), 126.6 (Ar), 125.7 (Ar), 114.3 (Ar), $103.8(\mathrm{CH}), 55.6\left(\mathrm{OCH}_{3}\right), 12.4\left(\mathrm{CH}_{3}\right)$. The spectra are in accordance with the literature data. ${ }^{3}$

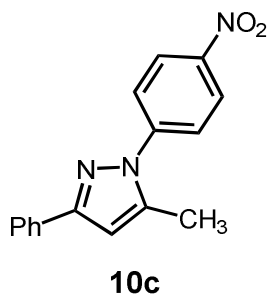


5-Methyl-1-(4-nitrophenyl)-3-phenyl-1H-pyrazole (10c). Prepared following General Procedure $\mathbf{C}$ using iron complex $7 \mathbf{b}$ (59 mg, $0.19 \mathrm{mmol}, 1.0$ equiv), and 4-nitrobenzenediazonium tetrafluoroborate ( $70 \mathrm{mg}, 0.29 \mathrm{mmol}, 1.5$ equiv). The reaction mixture was stirred at $60{ }^{\circ} \mathrm{C}$ for $12 \mathrm{~h}$ and the crude residue was purified by flash column chromatography (hexanes $/ \mathrm{EtOAc}=30: 1$ to 10:1). The desired product 10c was obtained as a white solid. Regioisomeric ratio $(>100: 1$ r.r.) was determined by ${ }^{1} \mathrm{H}$ NMR of the crude residue. Yield: $18 \mathrm{mg}, 35 \% .{ }^{1} \mathbf{H}$ NMR $\left(400 \mathrm{MHz}, \mathrm{CDCl}_{3}\right) \delta 8.38-8.35(\mathrm{~m}, 2 \mathrm{H})$, 7.87-7.85 (m, 2H), 7.81-7.77 (m, 2H), 7.45-7.41 (m, 2H), 7.38-7.34 (m, 1H), 6.61 (d, J=0.8 Hz, 1H), 2.52 (d, $J=0.4 \mathrm{~Hz}$, $3 \mathrm{H}) .{ }^{13} \mathrm{C}$ NMR $\left(100 \mathrm{MHz}, \mathrm{CDCl}_{3}\right) \delta 153.0(\mathrm{PhCN}), 145.9\left(\mathrm{CNO}_{2}\right), 145.0(\mathrm{Ar}), 140.6\left(\mathrm{CH}_{3} \underline{\mathrm{CN}}\right), 132.5(\mathrm{Ar}), 128.7(\mathrm{Ar})$, 128.4 (Ar), $125.8(\mathrm{Ar}), 124.8(\mathrm{Ar}), 123.9(\mathrm{Ar}), 106.6(\mathrm{CH}), 13.4\left(\mathrm{CH}_{3}\right)$. The spectra are in accordance with literature data. ${ }^{4}$

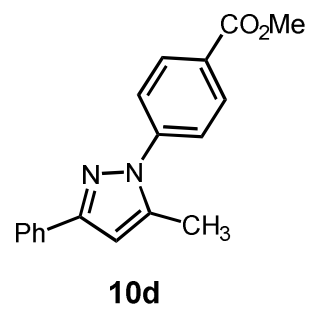

Methyl 4-(5-methyl-3-phenyl-1H-pyrazol-1-yl)benzoate (10d). Prepared following General Procedure $\mathbf{C}$ using iron complex $7 \mathbf{b}$ ( $53 \mathrm{mg}, 0.17 \mathrm{mmol}, 1.0$ equiv), and 4-(methoxycarbonyl)benzenediazonium tetrafluoroborate $(64 \mathrm{mg}, 0.25$ mmol, 1.5 equiv). The reaction mixture was stirred at $60^{\circ} \mathrm{C}$ for $12 \mathrm{~h}$ and the crude residue was purified by flash column chromatography (hexanes/EtOAc $=30: 1$ to 10:1). The desired product 10d was obtained as a white solid. Regioisomeric ratio (>100:1 r.r.) was determined by ${ }^{1} \mathrm{H}$ NMR of the crude residue. Yield: $17 \mathrm{mg}, 35 \% .{ }^{1} \mathbf{H} \mathbf{~ N M R}\left(500 \mathrm{MHz}, \mathrm{CDCl}_{3}\right) \delta$ 8.17-8.16 (m, 2H), 7.87-7.85 (m, 2H), $7.65(\mathrm{~d}, J=8.5 \mathrm{~Hz}, 2 \mathrm{H}), 7.43-7.40(\mathrm{~m}, 2 \mathrm{H}), 7.35-7.32(\mathrm{~m}, 1 \mathrm{H}), 6.57(\mathrm{~s}, 1 \mathrm{H}), 3.96$ (s, 3H), $2.46(\mathrm{~s}, 3 \mathrm{H}) .{ }^{13} \mathrm{C}$ NMR $\left(100 \mathrm{MHz}, \mathrm{CDCl}_{3}\right) \delta 166.4\left(\underline{\mathrm{CO}}_{2} \mathrm{Me}\right), 152.2(\mathrm{PhCN}), 143.6(\mathrm{Ar}), 140.3\left(\mathrm{CH}_{3} \underline{\mathrm{CN}}\right)$, $133.0(\mathrm{Ar}), 130.6(\mathrm{Ar}), 128.7(\mathrm{Ar}), 128.6(\mathrm{Ar}), 128.1(\mathrm{Ar}), 125.8(\mathrm{Ar}), 123.9(\mathrm{Ar}), 105.6(\mathrm{CH}), 52.3\left(\mathrm{CO}_{2} \underline{\mathrm{CH}_{3}}\right), 13.1$ $\left(\mathrm{CH}_{3}\right)$.

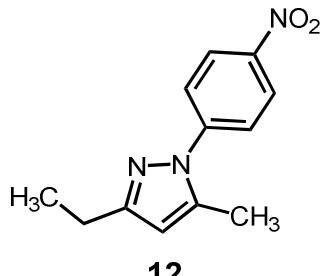

12

3-Ethyl-5-methyl-1-(4-nitrophenyl)-1H-pyrazole (12). Prepared following General Procedure E using iron complex 11 (49 mg, $0.19 \mathrm{mmol}, 1.0$ equiv), and 4-nitrobenzenediazonium tetrafluoroborate ( $70 \mathrm{mg}, 0.29 \mathrm{mmol}, 1.5$ equiv). The reaction mixture was stirred at $60{ }^{\circ} \mathrm{C}$ for $12 \mathrm{~h}$ and the crude residue was purified by flash column chromatography (hexanes $/ E t O A c=30: 1$ to 10:1). The desired product 12 was obtained as a yellow oil. Regioisomeric ratio (9.0:1 r.r.) was determined by GCMS. Yield: $17 \mathrm{mg}, 40 \% .{ }^{1} \mathbf{H}$ NMR $\left(400 \mathrm{MHz}, \mathrm{CDCl}_{3}\right) \delta 8.34-8.30(\mathrm{~m}, 2 \mathrm{H}), 7.70-7.67(\mathrm{~m}, 2 \mathrm{H}), 6.11(\mathrm{~s}$, $1 \mathrm{H}), 2.68(\mathrm{q}, J=7.6 \mathrm{~Hz}, 2 \mathrm{H}), 2.44(\mathrm{~d}, J=0.3 \mathrm{~Hz}, 3 \mathrm{H}), 1.28(\mathrm{t}, J=7.6 \mathrm{~Hz}, 3 \mathrm{H}) .{ }^{13} \mathbf{C} \mathbf{N M R}\left(100 \mathrm{MHz}, \mathrm{CDCl}_{3}\right) \delta 156.8$ $\left(\mathrm{CH}_{3} \mathrm{CH}_{2} \underline{\mathrm{CN}}\right), 145.6(\mathrm{Ar}), 145.1(\mathrm{Ar}), 139.8\left(\mathrm{CH}_{3} \underline{\mathrm{CN}}\right), 124.7(\mathrm{Ar}), 123.6(\mathrm{Ar}), 107.9(\mathrm{CH}), 21.5\left(\underline{\mathrm{CH}}_{2} \mathrm{CH}_{3}\right), 13.7$ $\left(\mathrm{CH}_{3}\right), 13.3\left(\mathrm{CH}_{3}\right)$. HRMS (ESI) calcd. for $\mathrm{C}_{12} \mathrm{H}_{14} \mathrm{~N}_{3} \mathrm{O}_{2}[\mathrm{M}+\mathrm{H}]^{+}:$232.1081, found 232.1084. Connectivity was assigned by nuclear Overhauser effect spectroscopy.

\subsection{Synthesis of $\mathrm{N}$-acylpyrazole 14}



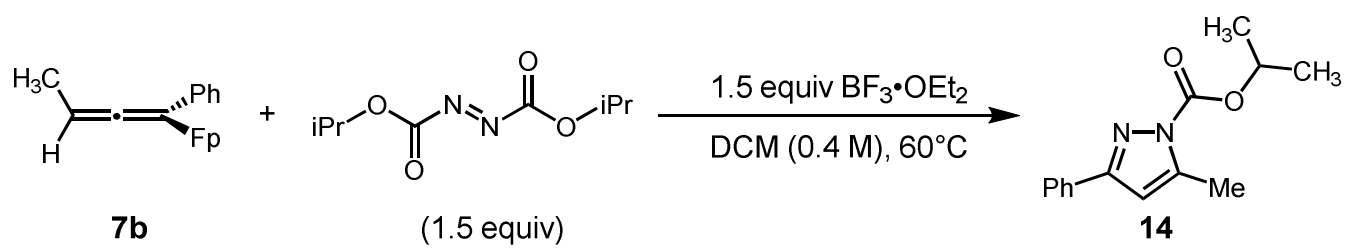

Isopropyl 5-methyl-3-phenyl-1H-pyrazole-1-carboxylate (14). In a glovebox, diisopropyl azodicarboxylate $(60 \mu \mathrm{L}$, $0.31 \mathrm{mmol}, 1.6$ equiv) and $\mathrm{BF}_{3} \cdot \mathrm{OEt}_{2}\left(38 \mu \mathrm{L}, 0.31 \mathrm{mmol}, 1.6\right.$ equiv) was dissolved $\mathrm{CH}_{2} \mathrm{Cl}_{2}(0.25 \mathrm{~mL})$ in a reaction tube equipped with a magnetic stir bar. A solution of allenyliron complex $7 \mathbf{b}\left(59 \mathrm{mg}, 0.19 \mathrm{mmol}, 1.0\right.$ equiv) in $\mathrm{CH}_{2} \mathrm{Cl}_{2}(0.25$ $\mathrm{mL}$ ) was added in rapid succession. The reaction mixture was stirred at $60^{\circ} \mathrm{C}$ for $12 \mathrm{~h}$ and the crude residue was purified by flash column chromatography (hexanes/EtOAc $=30: 1$ to 5:1). The desired product 14 was obtained as a brown oil. Regioisomeric ratio (> 20:1 r.r.) was determined by ${ }^{1} \mathrm{H}$ NMR of the crude residue. Yield: $17 \mathrm{mg}, 36 \% .{ }^{1} \mathbf{H}$ NMR (400 $\left.\mathrm{MHz}, \mathrm{CDCl}_{3}\right) \delta$ 7.87-7.85 (m, 2H), 7.43-7.34 (m, 3H), $6.48(\mathrm{~s}, 1 \mathrm{H}), 5.29-5.23(\mathrm{~m}, 1 \mathrm{H}), 2.59(\mathrm{~s}, 3 \mathrm{H}), 1.48(\mathrm{~d}, J=6.4 \mathrm{~Hz}$, 6H). ${ }^{13} \mathrm{C}$ NMR $\left(125 \mathrm{MHz}, \mathrm{CDCl}_{3}\right) \delta 153.9\left(\mathrm{CO}_{2} \mathrm{iPr}\right), 150.2(\mathrm{PhCN}), 145.1\left(\mathrm{CH}_{3} \underline{\mathrm{CN}}\right), 132.0(\mathrm{Ar}), 128.9(\mathrm{Ar}), 128.6$ (Ar), $126.5(\mathrm{Ar}), 107.7(\mathrm{CH}), 72.6\left(\underline{\mathrm{CH}}\left(\mathrm{CH}_{3}\right)_{2}\right), 21.8\left(\mathrm{CH}(\underline{\mathrm{CH}})_{2}\right), 14.7\left(\mathrm{CH}_{3}\right)$. HRMS (ESI) calcd. for $\mathrm{C}_{14} \mathrm{H}_{17} \mathrm{~N}_{2} \mathrm{O}_{2}$ $[\mathrm{M}+\mathrm{H}]^{+}: 245.1285$, found 245.1285 . Connectivity was verified by comparison with an independent synthesis of the pyrazole following literature report. ${ }^{5}$

Independent synthesis of 14:

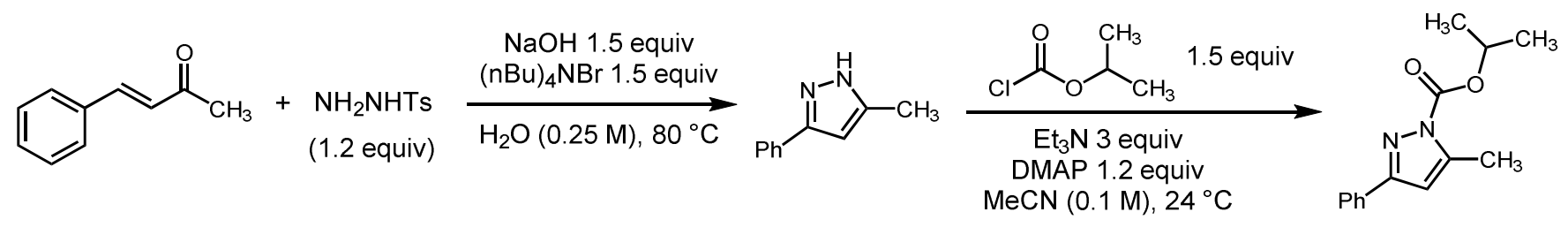

Step $1:{ }^{\mathrm{sa}}$ Into a reaction tube charged with (E)-4-phenylbut-3-en-2-one (73 mg, $0.50 \mathrm{mmol}, 1.0$ equiv), $p$-toluenesulfonyl hydrazide ( $111 \mathrm{mg}, 0.60 \mathrm{mmol}, 1.2$ equiv), tetrabutylammonium bromide ( $242 \mathrm{mg}, 0.75 \mathrm{mmol}, 1.5$ equiv) and $\mathrm{NaOH}(30$ $\mathrm{mg}, 0.75 \mathrm{mmol}, 1.5$ equiv) was added water $(2 \mathrm{~mL})$. The reaction mixture was stirred at $80^{\circ} \mathrm{C}$ under air for $10 \mathrm{~h}$. The reaction was allowed to cool to room temperature, diluted with $20 \mathrm{~mL}$ EtOAc. The organic phase was washed with brine and dried over $\mathrm{Na}_{2} \mathrm{SO}_{4}$. After the removal of the solvent, the crude residue was purified by flash column chromatography (hexanes/EtOAc $=10: 1)$. The desired product 5-methyl-3-phenyl-1H-pyrazole was obtained as a white solid. Yield: $65 \mathrm{mg}$, 82\%. ${ }^{1}$ H NMR (300 MHz, $\left.\mathrm{CDCl}_{3}\right)$ \& 7.73-7.70 (m, 2H), 7.43-7.38 (m, 2H), 7.34-7.30 (m, 1H), $6.37(\mathrm{~s}, 1 \mathrm{H}), 2.36(\mathrm{~s}, 3 \mathrm{H})$.

Step 2: Into a reaction tube charged with 5-methyl-3-phenyl-1H-pyrazole $(65 \mathrm{mg}, 0.41 \mathrm{mmol}, 1.0 \mathrm{equiv})$ was added $\mathrm{MeCN}(4 \mathrm{~mL})$, followed by $\mathrm{Et}_{3} \mathrm{~N}(171 \mu \mathrm{L}, 1.23 \mathrm{mmol}, 3.0$ equiv) and 4-(dimethylamino)pyridine (60 mg, $0.49 \mathrm{mmol}, 1.2$ equiv). The reaction was then cooled to $0{ }^{\circ} \mathrm{C}$, and isopropyl chloroformate ( $1 \mathrm{M}$ in toluene, $620 \mu \mathrm{L}, 0.62 \mathrm{mmol}, 1.5$ equiv) was added dropwise. The reaction was then warmed to room temperature and stirred for $2 \mathrm{~h}$. After the removal of the solvent, the crude residue was purified by flash column chromatography (hexanes/EtOAc $=10: 1$ ). Only one spot was appeared on the TLC plate and the product was obtained as a colorless oil. Yield: $40 \mathrm{mg}, 40 \% .{ }^{1} \mathbf{H} \mathbf{~ N M R}\left(300 \mathrm{MHz}, \mathrm{CDCl}_{3}\right)$ $\delta$ 7.87-7.85 (m, 2H), 7.44-7.35 (m, 3H), $6.47(\mathrm{~s}, 1 \mathrm{H})$, 5.30-5.22 (m, $1 \mathrm{H}), 2.59(\mathrm{~s}, 3 \mathrm{H}), 1.48(\mathrm{~d}, J=6.3 \mathrm{~Hz}, 6 \mathrm{H})$. The spectrum was found to be indistinguishable from that of $\mathbf{1 4}$ prepared from the iron complex.

In a previous report, the acylation of 3-methyl-5-phenyl-1H-pyrazole (which should rapidly tautomerize with 5-methyl-3phenyl-1H-pyrazole in solution at room temperature $)^{\text {sc }}$ gave 3-methyl-5-phenyl- and 5-methyl-3-phenyl-1-acetylpyrazole as a 1:5 mixture. ${ }^{5 b}$ The spectrum we recorded was clearly analogous to that of 5-methyl-3-phenyl-1-acetylpyrazole rather than that of 3-methyl-5-phenyl-1-acetylpyrazole $\left(\mathrm{CDCl}_{3}, 2.60 \mathrm{ppm} v s .2 .30 \mathrm{ppm}\right.$ for the methyl group on the pyrazole, and $6.50 \mathrm{ppm} v s .6 .17 \mathrm{ppm}$ for the pyrazole hydrogen). ${ }^{5 b} \mathrm{In}$ addition, the $\mathrm{N}$-acylation is believed to be a reversible process and sterically less hindered derivative is expected to be the favored product. ${ }^{\text {sd }}$ Thus, we assign the final acylation product as isopropyl 5-methyl-3-phenyl-1H-pyrazole-1-carboxylate. 


\section{Mechanistic studies}

\subsection{Kinetic study}

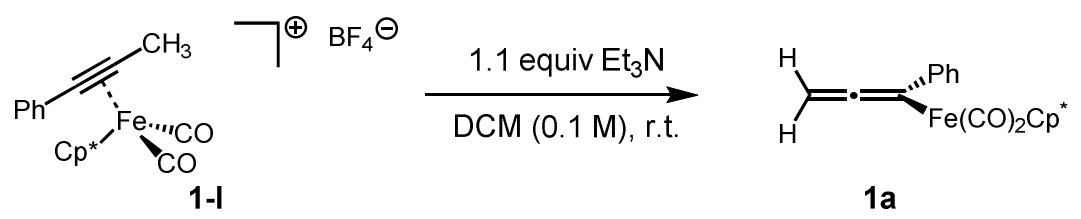

To a solution of $\eta^{2}$-1-phenyl-1-propyne complex 1-I $\left(22.5 \mathrm{mg}, 0.05 \mathrm{mmol}, 1.0\right.$ equiv) in dry $\mathrm{CH}_{2} \mathrm{Cl}_{2}(0.5 \mathrm{~mL})$ was added $\mathrm{Et}_{3} \mathrm{~N}$ ( $8 \mu \mathrm{L}, 0.055 \mathrm{mmol}, 1.1$ equiv) in an argon filled glovebox with the reaction vessel was protected from light. The mixture was stirred at room temperature for $1 \mathrm{~min}$. Then, the solvent was removed under reduced pressure within an additional $2 \mathrm{~min}$, the residue was suspended in $\operatorname{dry~}_{\mathrm{Et}} \mathrm{O}(8 \mathrm{~mL})$, and the suspension was filtered through Celite to remove the insoluble triethylammonium tetrafluoroborate. The filtrate was concentrated in vacuo. In an argon filled glovebox, protected from light, the allenyliron complex $1 \mathrm{a}$ was dissolved in $\mathrm{CD}_{2} \mathrm{Cl}_{2}(0.6 \mathrm{~mL})$ and the solution was added to an NMR tube charged with [2.2] paracyclophane as the internal standard. The NMR was then sealed with Parafilm, wrapped with aluminum foil and moved out of the glovebox for subsequent quantitative ${ }^{1} \mathrm{H}$ NMR spectroscopy.

Table S1: Kinetic analysis of $\eta^{1}$-allenyliron to $\eta^{1}$-propargyliron isomerization

\begin{tabular}{ccccc}
\hline $\begin{array}{c}\text { Time } \\
(\mathrm{min})\end{array}$ & $\begin{array}{c}\text { allenyliron] } \\
(\mathrm{M})\end{array}$ & $\begin{array}{c}\text { [propargyliron] } \\
(\mathrm{M})\end{array}$ & $\ln ([$ allenyliron] $)$ & Fraction of propargyliron \\
\hline 0 & 0.205 & 0.001 & 1.451 & 0.007 \\
59 & 0.199 & 0.003 & 1.425 & 0.017 \\
122 & 0.193 & 0.005 & 1.393 & 0.028 \\
179 & 0.192 & 0.007 & 1.389 & 0.034 \\
299 & 0.190 & 0.009 & 1.378 & 0.045 \\
539 & 0.186 & 0.012 & 1.354 & 0.063 \\
1325 & 0.185 & 0.019 & 1.347 & 0.093 \\
1816 & 0.166 & 0.023 & 1.238 & 0.122 \\
1975 & 0.160 & 0.024 & 1.206 & 0.130 \\
2799 & 0.138 & 0.030 & 1.057 & 0.179 \\
3416 & 0.135 & 0.041 & 1.036 & 0.235 \\
4204 & 0.119 & 0.057 & 0.907 & 0.323 \\
\hline
\end{tabular}

\subsection{Effect of additives}

To a solution of $\eta^{2}$-1-phenyl-1-propyne complex 1-I ( $22.5 \mathrm{mg}, 0.05 \mathrm{mmol}, 1.0$ equiv) in dry $\mathrm{CH}_{2} \mathrm{Cl}_{2}(0.5 \mathrm{~mL})$ was added $\mathrm{Et}_{3} \mathrm{~N}$ ( $8 \mu \mathrm{L}, 0.055 \mathrm{mmol}, 1.1$ equiv) outside of the glovebox. The mixture was stirred at room temperature for $1 \mathrm{~min}$. Then, the solvent was removed under reduced pressure within an additional $2 \mathrm{~min}$, the residue was suspended in $\operatorname{dry} \mathrm{Et}_{2} \mathrm{O}(8$ $\mathrm{mL}$ ), and the suspension was filtered through Celite to remove the insoluble triethylammonium tetrafluoroborate. The filtrate was concentrated in vacuo. The allenyliron complex 1 a was dissolved in $0.5 \mathrm{~mL} \mathrm{CD}_{2} \mathrm{Cl}_{2}$, and the solution was added to NMR tube charged with $\left[\mathrm{Et}_{3} \mathrm{NH}\right]^{+}\left[\mathrm{BF}_{4}\right]^{-}\left(9.4 \mathrm{mg}, 1.0\right.$ equiv), $\mathrm{Et}_{3} \mathrm{~N}$ (7 $\mu \mathrm{L}, 1.0$ equiv) or $\mathrm{Fp}^{*}(\mathrm{thf}) \mathrm{BF}_{4}(3 \mathrm{mg}, 15 \%)$ for subsequent quantitative ${ }^{1} \mathrm{H}$ NMR spectroscopy. 
Table S2: Comparison of the fraction of $\boldsymbol{\eta}^{1}$-propargyliron after adding additives

\begin{tabular}{cc|cc|cc}
\hline \multicolumn{2}{c|}{$15 \% \mathrm{Fp}^{*}(\mathrm{thf}) \mathrm{BF}_{4}$} & \multicolumn{2}{|c|}{1.0 equiv $\mathrm{Et}_{3} \mathrm{NHBF}_{4}$} & \multicolumn{2}{c}{1.0 equiv $\mathrm{Et}_{3} \mathrm{~N}$} \\
\hline time & & time & time & \\
$(\mathrm{min})$ & Fraction of propargyliron & $(\mathrm{min})$ & Fraction of propargyliron & $(\mathrm{min})$ & Fraction of propargyliron \\
0 & 0.156 & 0 & 0.006 & 0 & 0.000 \\
35 & 0.349 & 36 & 0.056 & 29 & 0.000 \\
65 & 0.425 & 92 & 0.151 & 94 & 0.000 \\
121 & 0.517 & 159 & 0.267 & 208 & 0.000 \\
244 & 0.616 & 339 & 0.559 & 332 & 0.000 \\
1110 & 0.982 & 517 & 0.743 & 567 & 0.018 \\
& & & & 1344 & 0.059 \\
\hline
\end{tabular}

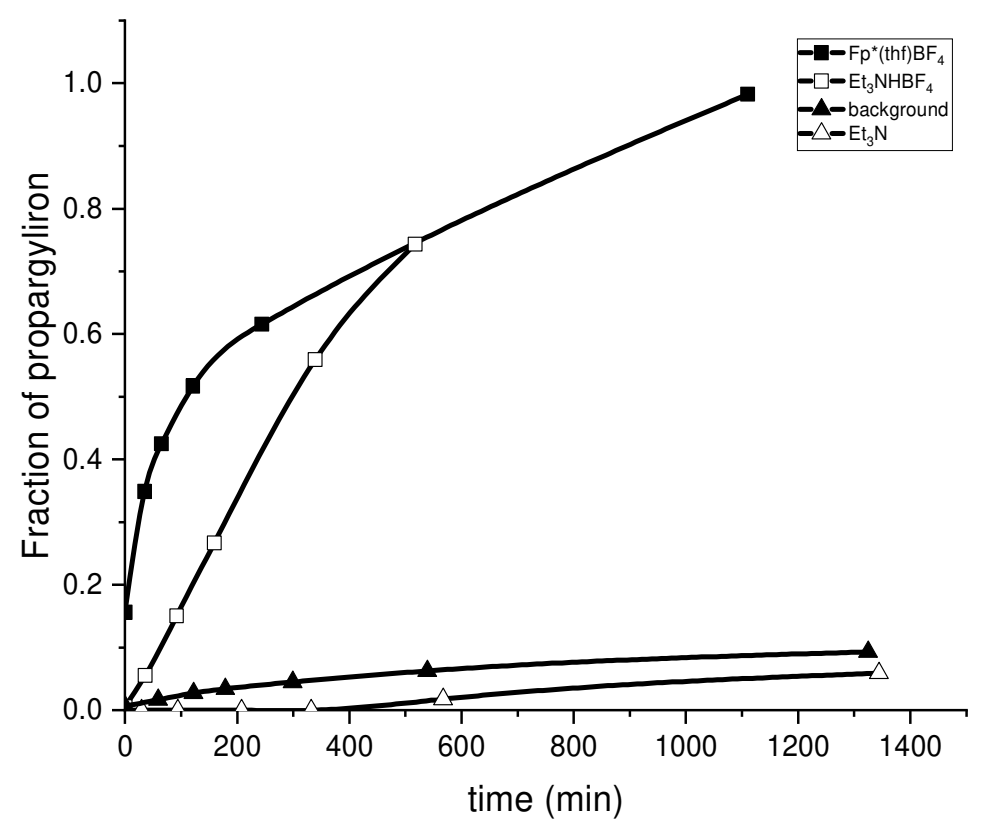

Figure S1: Plot of the fraction of $\eta^{1}$-propargyliron vs time

\subsection{Effect of temperature}

To a solution of $\eta^{2}$-1-phenyl-1-propyne complex 1-I $\left(22.6 \mathrm{mg}, 0.05 \mathrm{mmol}, 1.0\right.$ equiv) in dry $\mathrm{CH}_{2} \mathrm{Cl}_{2}(0.5 \mathrm{~mL}, 0.1 \mathrm{M})$ was added $\mathrm{Et}_{3} \mathrm{~N}$ ( $8.3 \mu \mathrm{L}, 0.06 \mathrm{mmol}, 1.2$ equiv) outside of the glove box. The mixture was stirred at room temperature for 1 $\mathrm{min}$. Then, the solvent was removed under reduced pressure within an additional $2 \mathrm{~min}$, the residue was suspended in dry $\mathrm{Et}_{2} \mathrm{O}(10 \mathrm{~mL})$, and the suspension was filtered through Celite to remove the insoluble triethylammonium tetrafluoroborate. The filtrate was concentrated in vacuo at $20^{\circ} \mathrm{C} .2500 \mu \mathrm{L}$ of $\mathrm{C}_{6} \mathrm{D}_{6}$ was added to make the stock solution. In each separated NMR tube, $500 \mu \mathrm{L}$ stock solution of $1 \mathrm{a}$ was added. The NMR tubes were placed into an oil bath which was preheated and stabilized at the desired temperature for $60 \mathrm{~min}$. Then the NMR tubes were taken out of the oil bath for subsequent ${ }^{1}$ HNMR spectroscopy. The conversion to $1 \mathbf{a}^{\prime}$ stabilizes at approximatively $92 \%$, suggesting the equilibrium is reached at that point. 


\begin{tabular}{cc}
\hline Temperature $\left({ }^{\circ} \mathrm{C}\right)$ & Fraction of propargyliron \\
\hline 24.0 & 0.013 \\
33.7 & 0.140 \\
37.6 & 0.443 \\
52.8 & 0.760 \\
61.0 & 0.846 \\
61.0 & $0.920(1.5 \mathrm{~h})$ \\
66.6 & $0.921(2.0 \mathrm{~h})$ \\
\hline
\end{tabular}

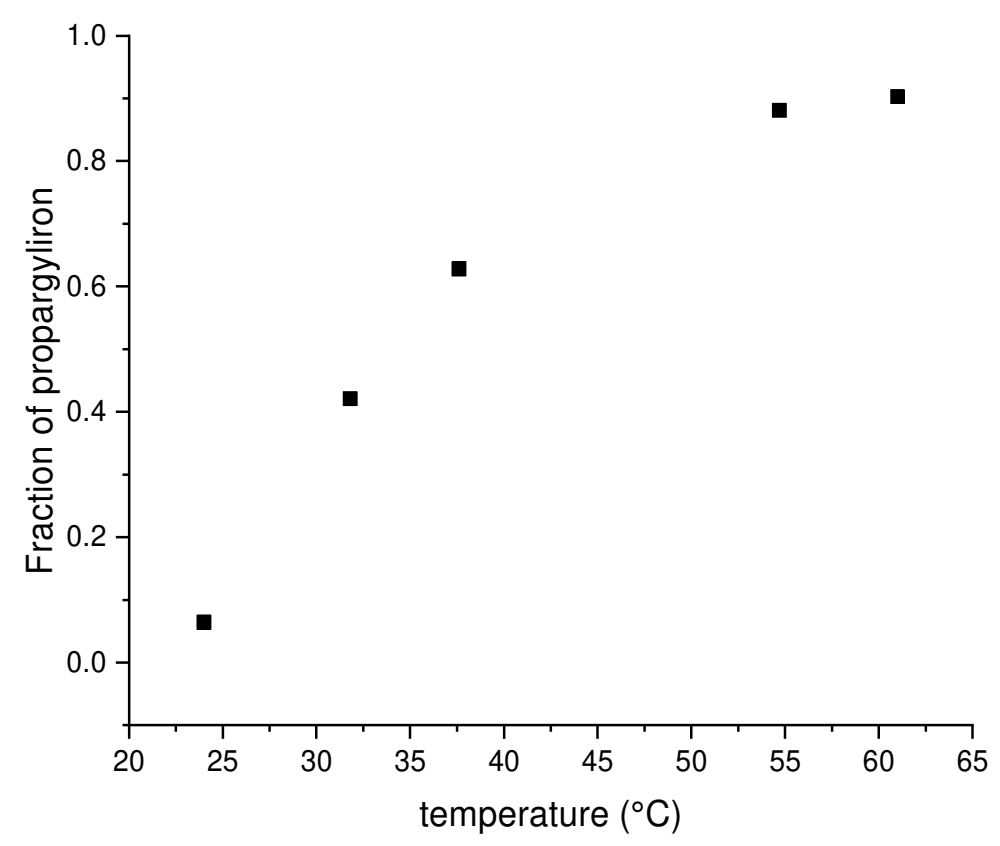

Figure S2: Plot of the fraction of $\eta^{1}$-propargyliron vs temperature

\subsection{Effect of irradiation}

To a solution of $\eta^{2}$-1-phenyl-1-propyne complex 1-I $\left(27.2 \mathrm{mg}, 0.06 \mathrm{mmol}, 1.0\right.$ equiv) in dry $\mathrm{CH}_{2} \mathrm{Cl}_{2}(0.6 \mathrm{~mL}, 0.1 \mathrm{M})$ was added $\mathrm{Et}_{3} \mathrm{~N}(10 \mu \mathrm{L}, 0.066 \mathrm{mmol}, 1.1$ equiv) outside of the glovebox. The mixture was stirred at room temperature for 1 $\mathrm{min}$. Then, the solvent was removed under reduced pressure within an additional $2 \mathrm{~min}$, the residue was suspended in dry $\mathrm{Et}_{2} \mathrm{O}(10 \mathrm{~mL})$, and the suspension was filtered through Celite to remove the insoluble triethylammonium tetrafluoroborate. The filtrate was concentrated in vacuo. The allenyliron complex $1 \mathrm{a}$ was dissolved in $\mathrm{C}_{6} \mathrm{D}_{6}(1.2 \mathrm{~mL})$, and $0.6 \mathrm{~mL}$ of the stock solution was transferred to two NMR tube separately. Each NMR tube was flushed with nitrogen. Into one of the NMR tubes was added $\mathrm{Fp}_{2}{ }_{2}(10 \mu \mathrm{L}$ of $0.03 \mathrm{M}$ stock solution, $1 \%)$ via a syringe. The two NMR tubes were placed inside an $85 \mathrm{~W}$ CFL for $15 \mathrm{~min}$. The mixture was filtered over cotton to remove insoluble matter. The solution was then transfer to a clean NMR tube and flushed with nitrogen for subsequent ${ }^{1} \mathrm{H}-\mathrm{NMR}$ spectroscopy. On standing in $\mathrm{C}_{6} \mathrm{D}_{6}$, a loss of resolution due to paramagnetic broadening, as well as the formation of a solid residue were observed after $12 \mathrm{~h}$, indicating the decomposition of the product 1a". CO gas was introduced into a reaction tube capped with a Teflon/silicon septum screw

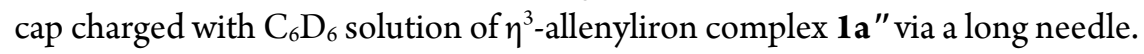




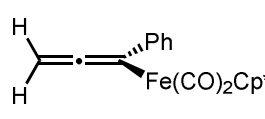

$1 a$

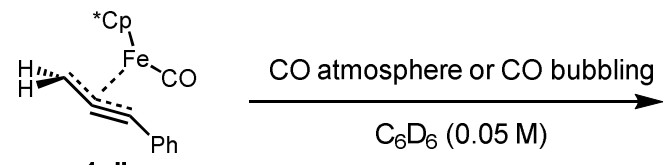

1a"
$\mathrm{C}_{6} \mathrm{D}_{6}(0.05 \mathrm{M})$ 85 W CFL, $15 \mathrm{~min}$

$\mathrm{C}_{6} \mathrm{D}_{6}(0.05 \mathrm{M})$

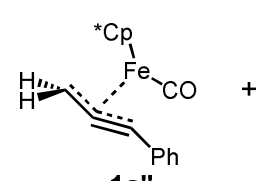

$1 \mathrm{a"}$

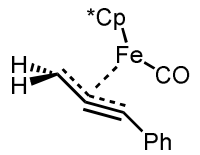

1a", quantitative

no detectable conversion

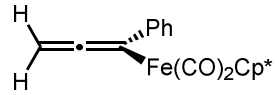

$1 \mathrm{a}$

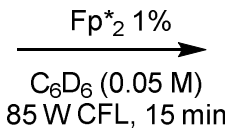

$85 \mathrm{WCFL}, 15 \mathrm{~min}$

14.4

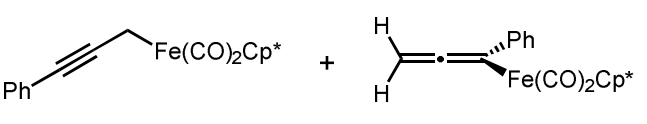

$1 a^{\prime}$

3.0
0.7

Scheme S1: Effect of irradiation on the $\eta^{1}$-allenyliron to $\eta^{1}$-propargyliron isomerization

Upon irradiation with $85 \mathrm{~W}$ CFL or $5 \mathrm{~W}$ blue LED for $15 \mathrm{~min}, \eta^{1}$-allenyliron complex $1 \mathrm{a}$ in $\mathrm{C}_{6} \mathrm{D}_{6}$ readily underwent decarbonylation to give the $\eta^{3}$-allenyl complex 1a" (Scheme S1). The ${ }^{1} \mathrm{H}$ NMR of $\mathbf{1} \mathbf{a}^{\prime \prime}$ in $\mathrm{C}_{6} \mathrm{D}_{6}$ exhibited a downfield-shifted $\mathrm{Cp}^{*}$ signal at $\delta 1.47 \mathrm{ppm}$ compared with that of allenyliron at $\delta 1.38 \mathrm{ppm}$. Two doublets at $\delta 2.81$ and $3.37 \mathrm{ppm}$ were assigned to the inequivalent hydrogens coupled to each other $\left(J_{\text {gem }}=8.5 \mathrm{~Hz}\right)$. In the $\mathrm{C}-\mathrm{H}$ coupled ${ }^{13} \mathrm{C}$ NMR spectrum of 1a", a doublet of doublets at $37.0 \mathrm{ppm}\left(J_{\mathrm{C}-\mathrm{H}}=163 \mathrm{~Hz}, 155 \mathrm{~Hz}\right)$ was assigned to the terminal allenyl carbons. In the C-H decoupled ${ }^{13} \mathrm{C}$ NMR, two signals at $\delta 95.6$ and $72.9 \mathrm{ppm}$ were assigned to the quaternary allenyl carbon, and two signals at $\delta 89.9$ and $9.4 \mathrm{ppm}$ were assigned to $\mathrm{Cp}^{*}$ moiety. The allenyl carbon chemical shifts as well as coupling constants of $\eta^{3}$ allenyl complex were similar to those previous reports of $\eta^{3}$-allenyl or $\eta^{3}$-propargyl complexes. ${ }^{6}$ In the IR spectrum of $\mathbf{1 a}$ " in toluene, one $\mathrm{CO}$ band was observed at $1915 \mathrm{~cm}^{-1}$. By comparison, complexes of analogous $\eta^{3}$-allyl complexes typically exhibit a single $v(\mathrm{CO})$ peak at 1950-1940 $\mathrm{cm}^{-1}$. Further introduction of $\mathrm{CO}$ into the $\mathrm{C}_{6} \mathrm{D}_{6}$ solution of $\mathbf{1 a}$ " indicated the irreversibility of the decarbonylation, indicating that although the addition of $\mathrm{Fp}_{2}{ }_{2}$ induced $\eta^{1}$-allenyliron (1a) to $\eta^{1}$ propargyliron $\left(\mathbf{1} \mathbf{a}^{\prime}\right)$ isomerization, the $\eta^{3}$-allenyl complex $\left(\mathbf{1} \mathbf{a}^{\prime \prime}\right)$ is probably not an intermediate.

Full characterization data for iron complex 1a':

${ }^{1} \mathbf{H} \operatorname{NMR}\left(400 \mathrm{MHz}, \mathrm{C}_{6} \mathrm{D}_{6}\right) \delta$ 7.59-7.56 (m, 2H), 7.07-7.03 (m, 2H), 6.98-6.94 (m, 1H), $1.60(\mathrm{~s}, 2 \mathrm{H}), 1.40(\mathrm{~s}, 15 \mathrm{H}) .{ }^{13} \mathbf{C}$ NMR $\left(100 \mathrm{MHz}, \mathrm{C}_{6} \mathrm{D}_{6}\right) \delta 218.0(\mathrm{CO}), 130.9(\mathrm{Ar}), 128.2(\mathrm{Ar}), 126.0(\mathrm{Ar}), 102.5(\underline{\mathrm{C}} \equiv \mathrm{C}), 95.0\left(\underline{\mathrm{C}}_{5} \mathrm{Me}_{5}\right), 81.1(\mathrm{C} \equiv \underline{\mathrm{C}}), 8.7$ $\left(\mathrm{C}_{5} \underline{\mathrm{Me}}_{5}\right),-12.2\left(\underline{\mathrm{CH}_{2} \mathrm{Fe}}\right)$ (one Ar-C peak was missing due to overlap). IR (ATR, $\mathrm{CCl}_{4}$ solution): $v(\mathrm{CO})=1986 \mathrm{~cm}^{-1}$, $1930 \mathrm{~cm}^{-1}$. 


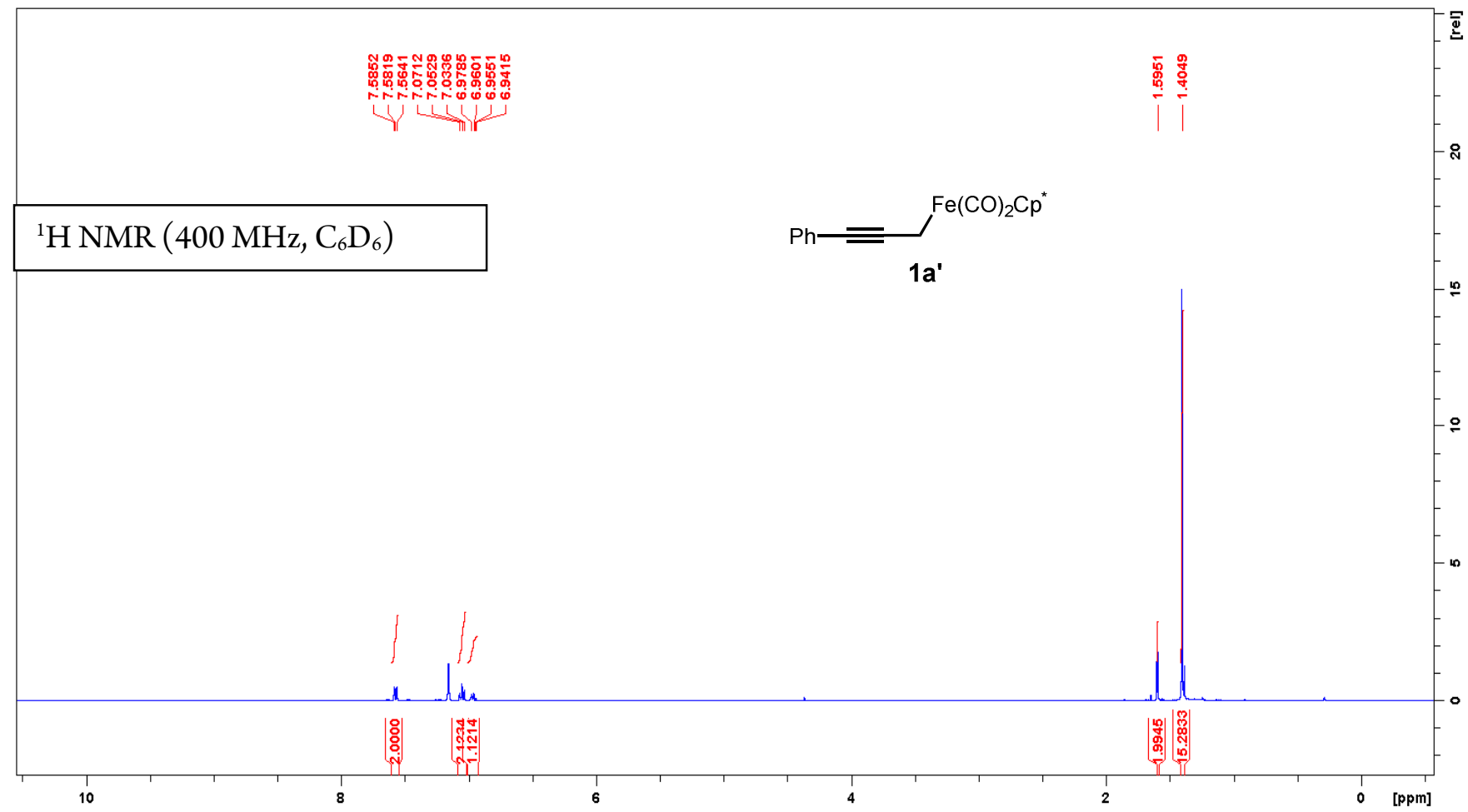

Figure S3: ${ }^{1} \mathrm{H}$ NMR spectrum of 1a'

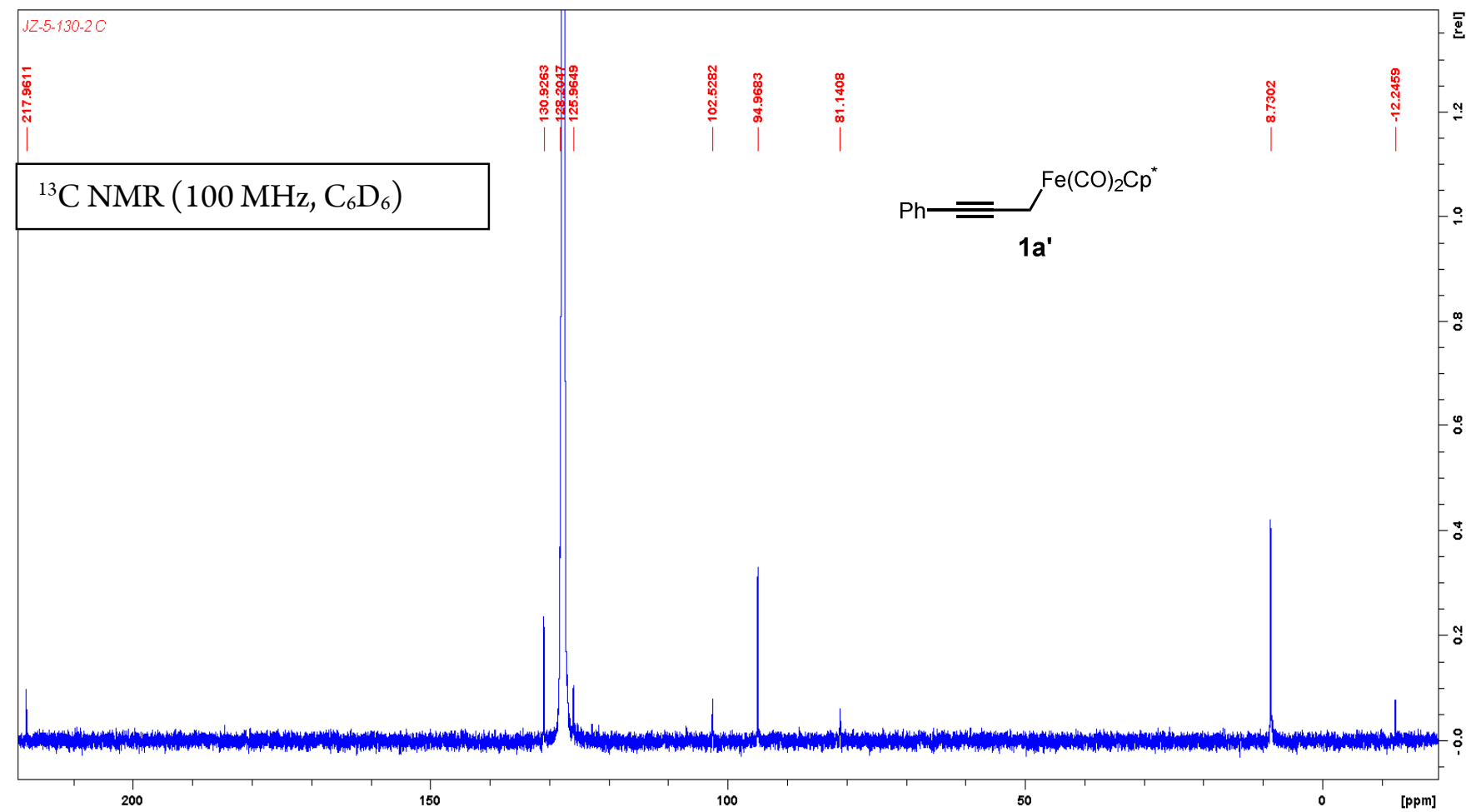

Figure S4: ${ }^{13} \mathrm{C}$ NMR spectrum of $1 a^{\prime}$ 


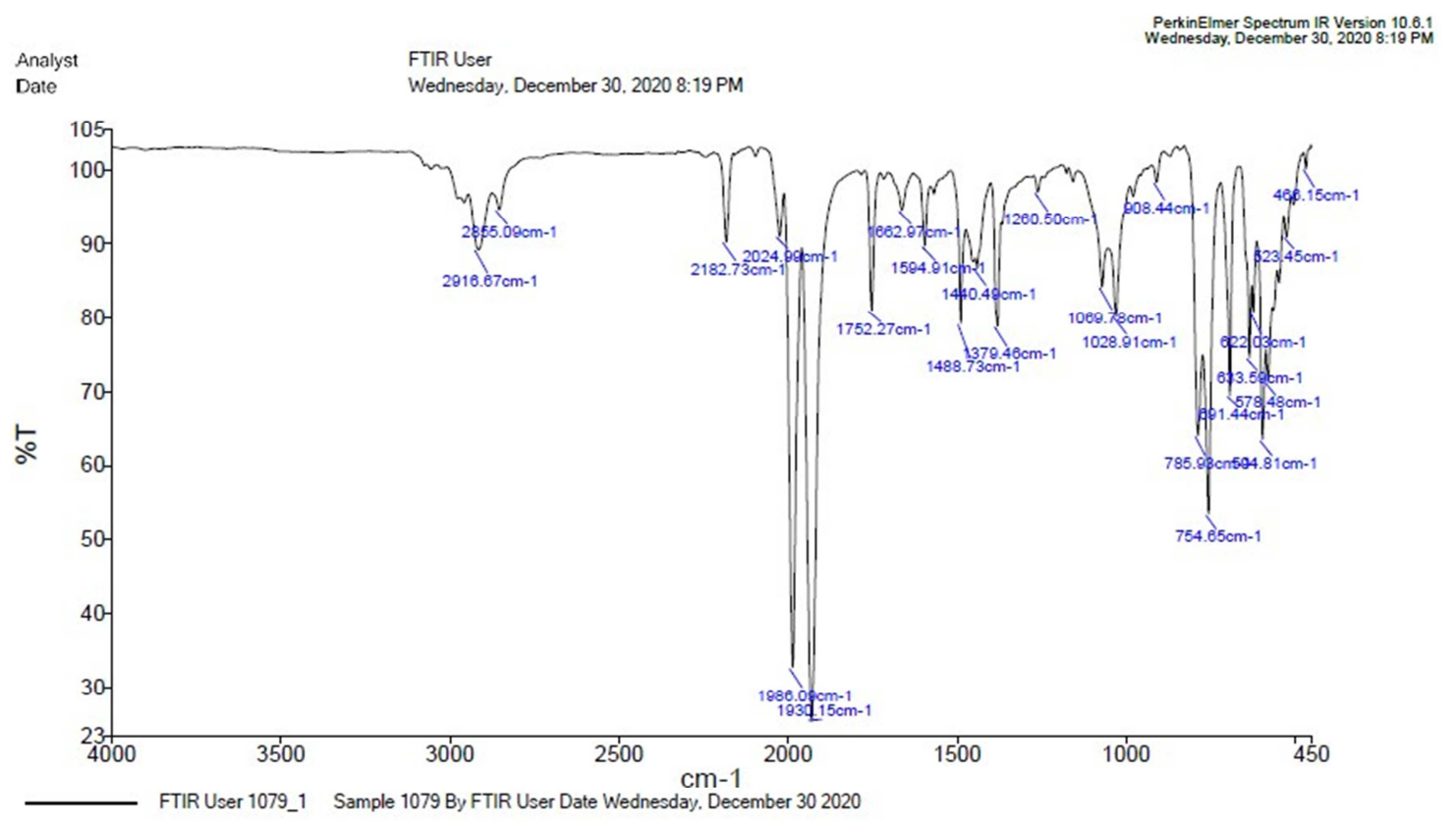

Figure S5: IR spectrum of 1a'

Full characterization data for iron complex 1a":

${ }^{1}$ H NMR $\left(500 \mathrm{MHz}, \mathrm{C}_{6} \mathrm{D}_{6}\right) \delta$ 7.82-7.80 (m, 2H), 7.17-7.15 (m, 2H, one Ar-H overlap with solvent residual peak), 7.07$7.04(\mathrm{~m}, 1 \mathrm{H}), 3.37(\mathrm{~d}, J=8.5 \mathrm{~Hz}, 1 \mathrm{H}), 2.81(\mathrm{~d}, J=8.5 \mathrm{~Hz}, 1 \mathrm{H}), 1.47(\mathrm{~s}, 15 \mathrm{H}) .{ }^{13} \mathbf{C} \mathbf{~ N M R}\left(125 \mathrm{MHz}, \mathrm{C}_{6} \mathrm{D}_{6}\right) \delta 215.4(\mathrm{CO})$, $131.8(\mathrm{Ar}), 131.2(\mathrm{Ar}), 128.2(\mathrm{Ar}), 126.6(\mathrm{Ar}), 95.6(\underline{\mathrm{C}}=\mathrm{C}), 89.9\left(\underline{\mathrm{C}}_{5} \mathrm{Me}_{5}\right), 72.9(\mathrm{C}=\underline{\mathrm{C}}), 36.9\left(\mathrm{dd}, J_{\mathrm{CH}}=163 \mathrm{~Hz}, 155 \mathrm{~Hz}\right.$, $\left.\mathrm{CH}_{2}\right)$, $9.4\left(\mathrm{C}_{5} \underline{\mathrm{Me}}_{5}\right)$. IR (ATR, toluene solution): $v(\mathrm{CO})=1915 \mathrm{~cm}^{-1}$.

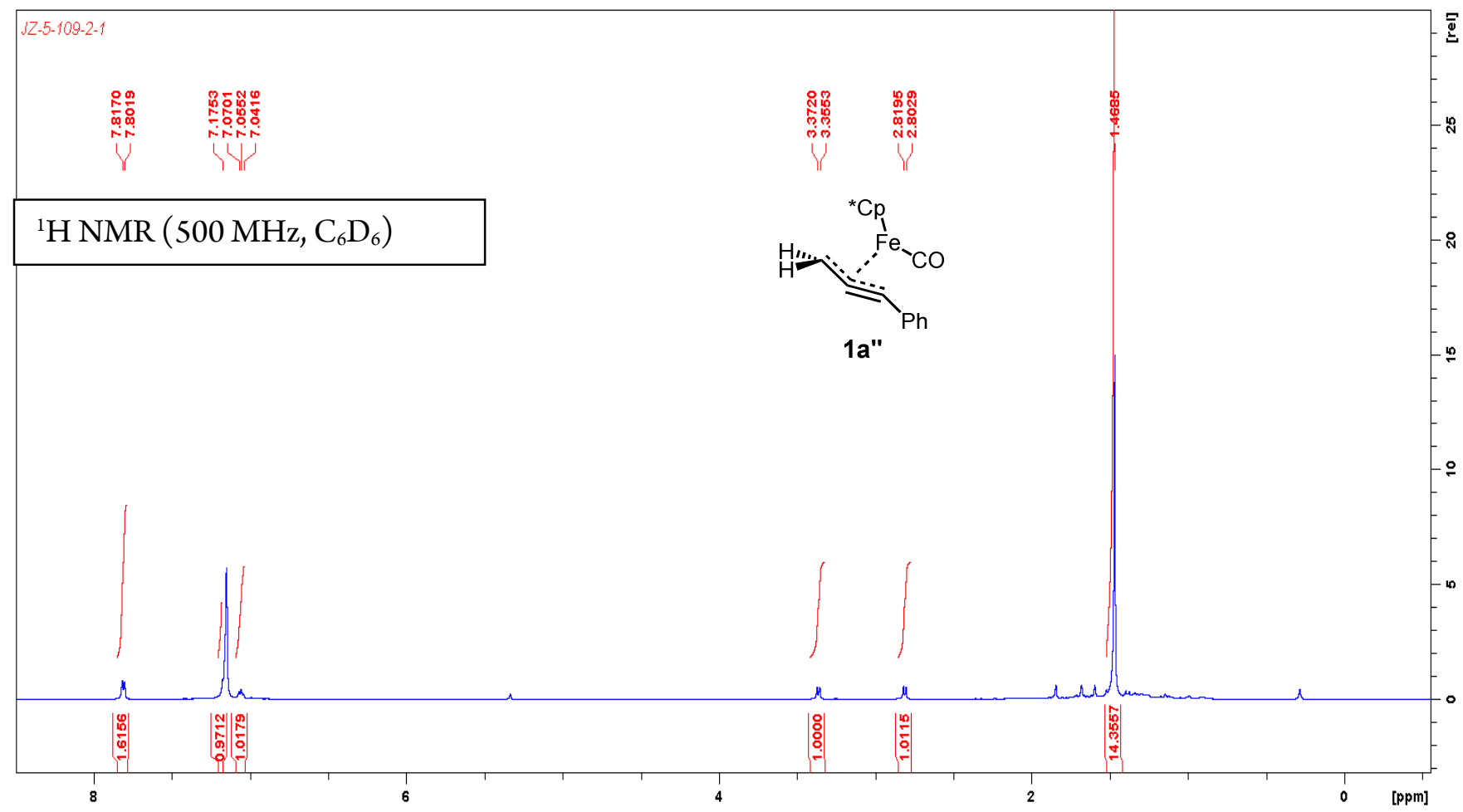

Figure S6: ${ }^{1} \mathrm{H}$ NMR spectrum of $1 \mathrm{a} "$ 


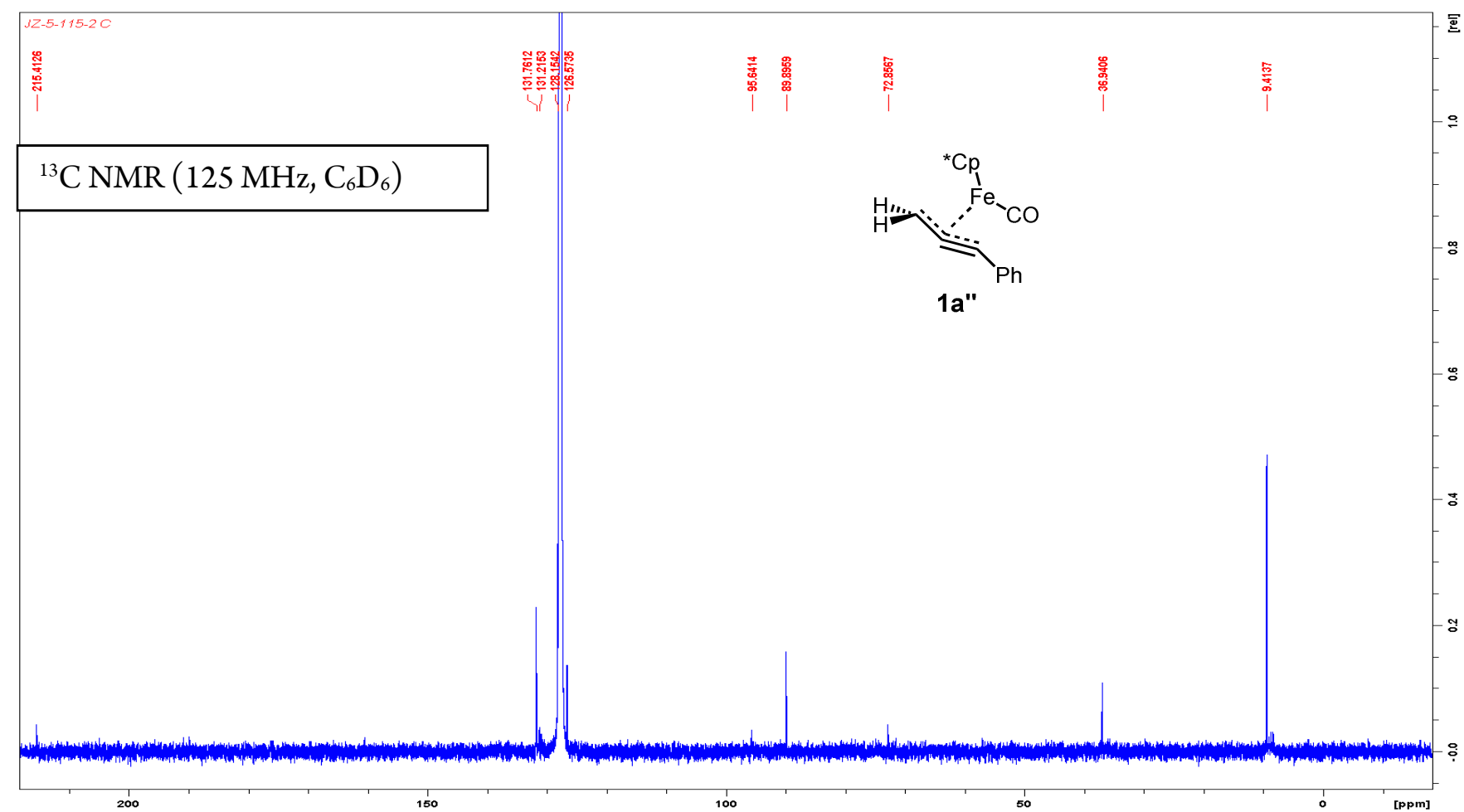

Figure S7: ${ }^{13} \mathrm{C}$ NMR spectrum of 1a"

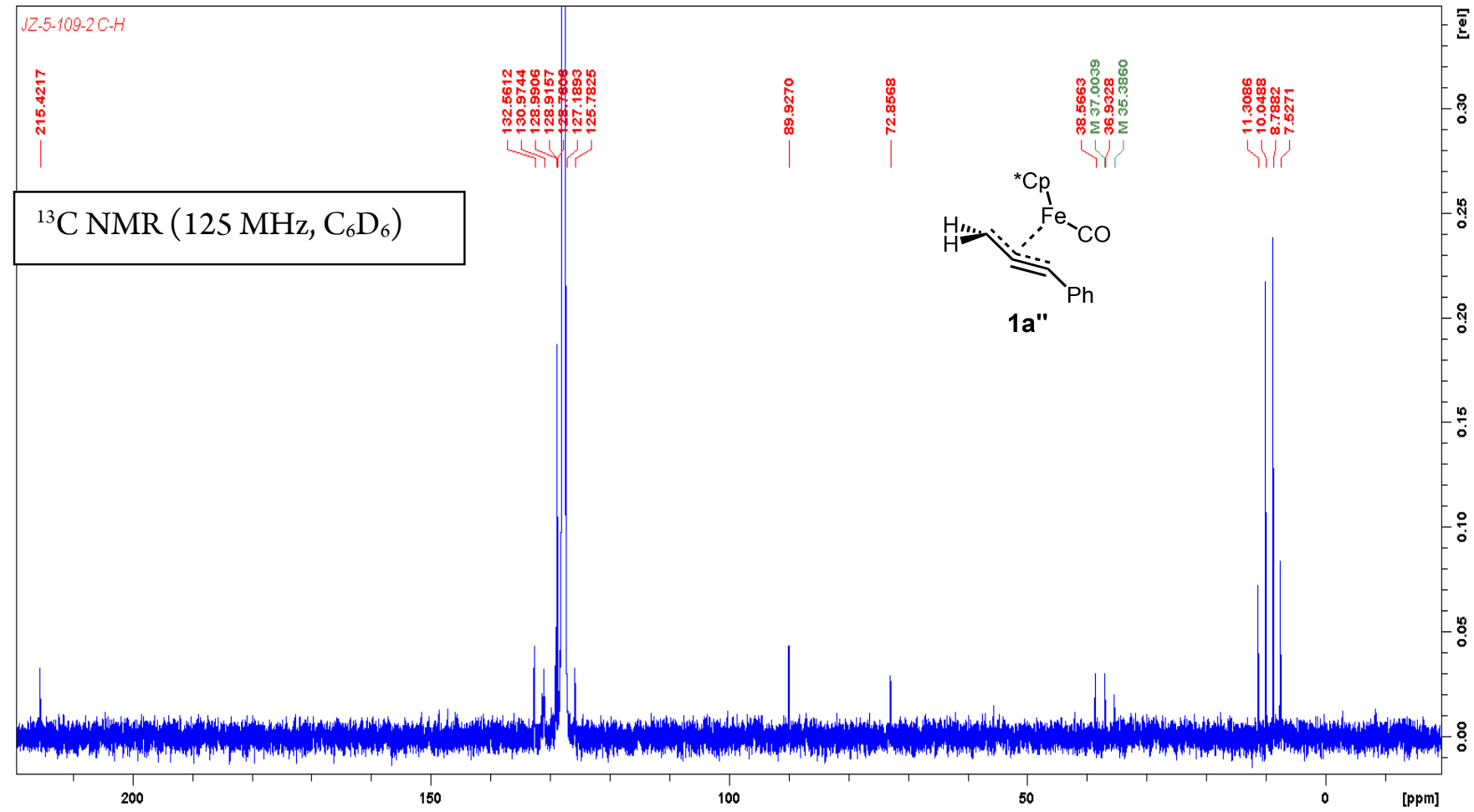

Figure S8: ${ }^{1} \mathrm{H}$-coupled ${ }^{13} \mathrm{C}$ NMR spectrum of 1a" 


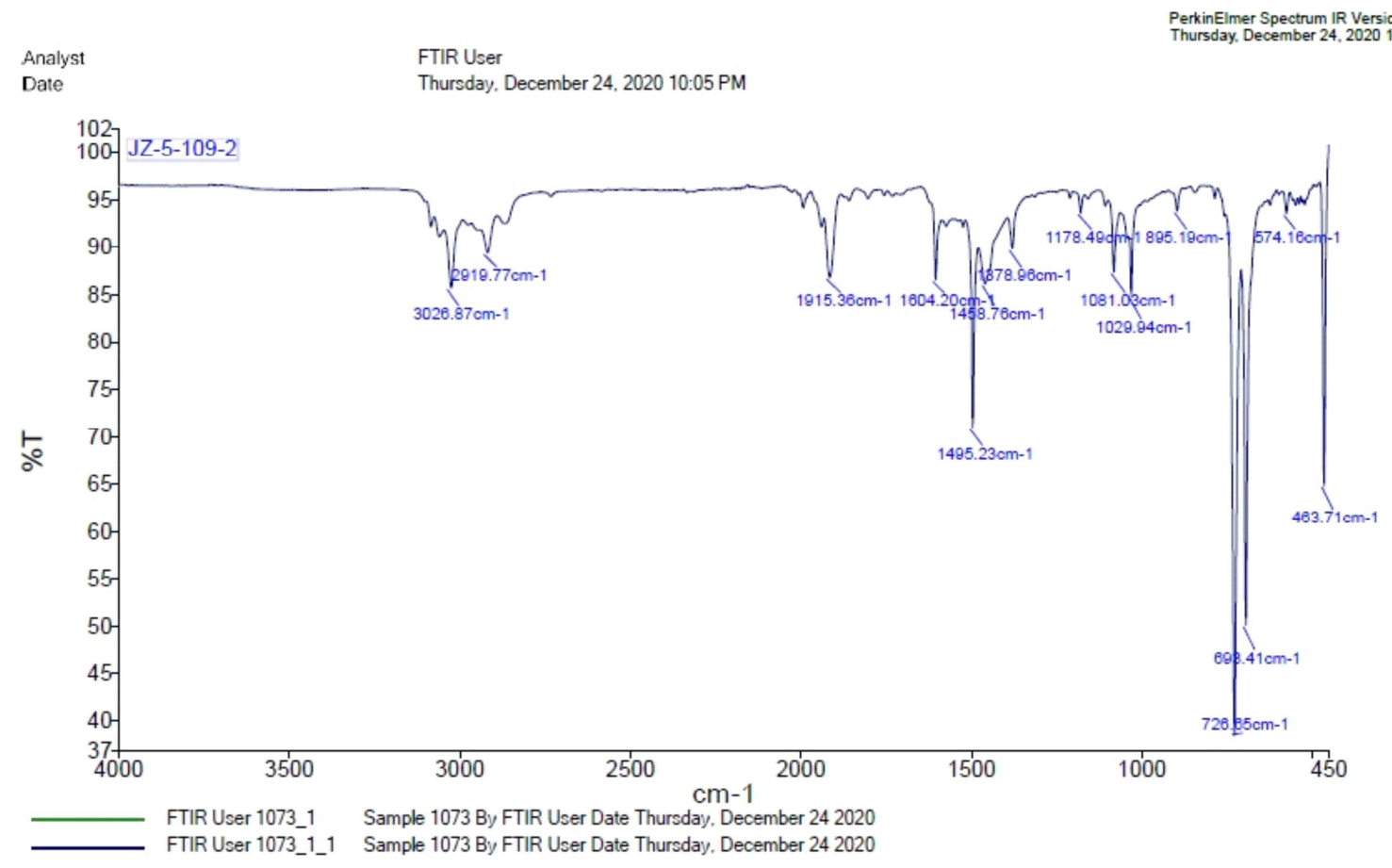

Figure S9: IR spectrum of 1a"

\subsection{Effect of added diazonium salt}

To a solution of $\eta^{2}$-1-phenyl-1-propyne complex 1-I $\left(13.6 \mathrm{mg}, 0.03 \mathrm{mmol}, 1.0\right.$ equiv) in dry $\mathrm{CH}_{2} \mathrm{Cl}_{2}(2 \mathrm{~mL}, 0.1 \mathrm{M})$ was added $\mathrm{Et}_{3} \mathrm{~N}$ ( $5 \mu \mathrm{L}, 0.033 \mathrm{mmol}, 1.1$ equiv) in an argon filled glovebox and the reaction vessel was protected from light. The mixture was stirred at room temperature for $1 \mathrm{~min}$. Then, the solvent was removed under reduced pressure within an addi-

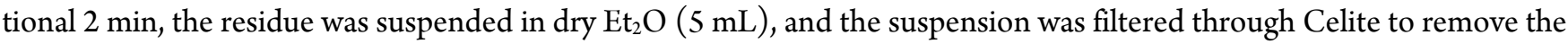
insoluble triethylammonium tetrafluoroborate. The filtrate was concentrated in vacuo. In an argon filled glovebox, the allenylrion complex $1 \mathrm{a}$ was dissolved in $0.6 \mathrm{~mL} \mathrm{C}_{6} \mathrm{D}_{6}$, and the solution was added to an NMR tube charged with phenyldiazonium tetrafluoroborate $(1.0 \mathrm{mg}, 10 \%)$. The NMR tube were moved out of the glovebox and put into an oil bath preheated to $40^{\circ} \mathrm{C}$ for $2 \mathrm{~h}$. The mixture was filtered over cotton into another NMR tube and flushed with nitrogen for subsequent ${ }^{1} \mathrm{H}$ NMR spectroscopy. With respect to rate of isomerization, the effect of added diazonium salt was found to be modest and somewhat inconsistent.

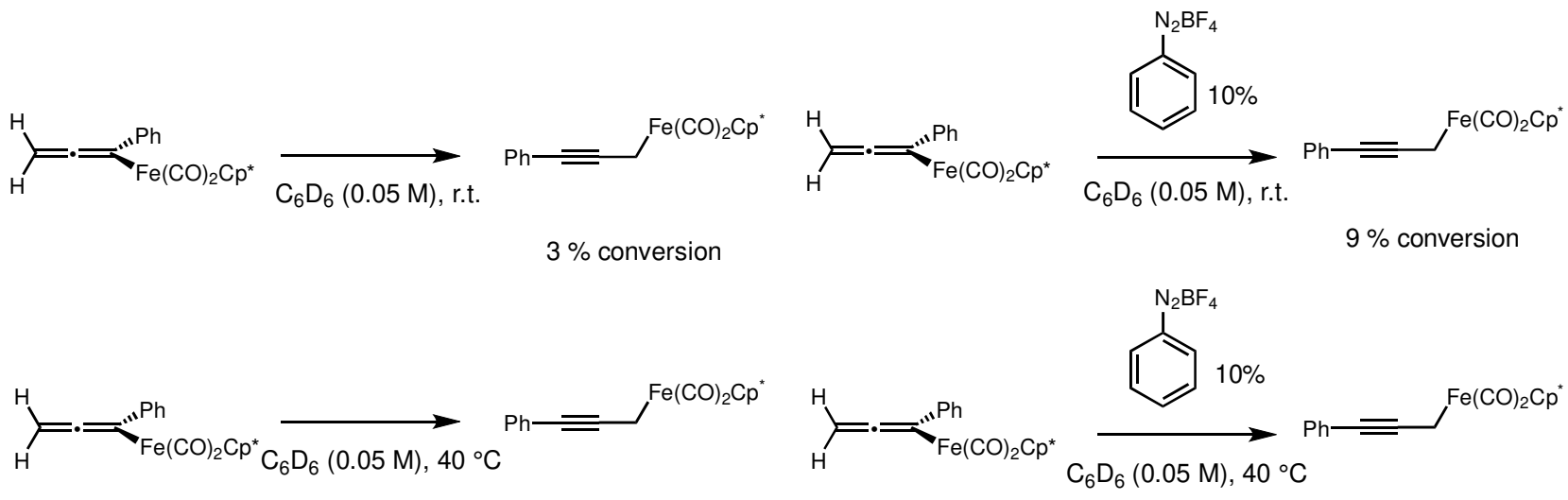

$86 \%$ conversion

$88 \%$ conversion

Scheme S2: Effect of diazonium on the $\eta^{1}$-allenyliron to $\eta^{1}$-propargyliron isomerization 
To a solution of $\eta^{2}$-1-phenyl-1-propyne complex 1-I $\left(23.0 \mathrm{mg}, 0.05 \mathrm{mmol}, 1.0\right.$ equiv) in dry $\mathrm{CH}_{2} \mathrm{Cl}_{2}(0.5 \mathrm{~mL}, 0.1 \mathrm{M})$ was added $\mathrm{Et}_{3} \mathrm{~N}$ (10 $\mu \mathrm{L}, 0.06 \mathrm{mmol}, 1.2$ equiv) outside of the glovebox. The mixture was stirred at room temperature for 1 $\mathrm{min}$. Then, the solvent was removed under reduced pressure within an additional $2 \mathrm{~min}$, the residue was suspended in dry $\mathrm{Et}_{2} \mathrm{O}(10 \mathrm{~mL})$, and the suspension was filtered through Celite to remove the insoluble triethylammonium tetrafluoroborate. The filtrate was concentrated in vacuo. The allenyliron complex 1 a was dissolved in $2.0 \mathrm{~mL} \mathrm{C}_{6} \mathrm{D}_{6}$ in the glovebox. Into one NMR tube charged with benzoyl peroxide was added $0.5 \mathrm{~mL}$ of the stock solution, and into two other NMR tubes were added $0.5 \mathrm{~mL}$ of the stock solution. The three NMR tubes were moved out of the glovebox, and into one of the NMR tubes containing $0.5 \mathrm{~mL}$ stock solution was purged with $\mathrm{O}_{2}$ with a balloon through a long needle for $5 \mathrm{~min}$. The three NMR tubes were placed close to each other into an oil bath preheated at $51.0^{\circ} \mathrm{C}$ for $60 \mathrm{~min}$. Then the NMR tube was taken out of the oil bath for subsequent ${ }^{1} \mathrm{H}$ NMR spectroscopy.

To a solution of $\eta^{2}$-1-phenyl-1-propyne complex 1-I $\left(23.0 \mathrm{mg}, 0.05 \mathrm{mmol}, 1.0\right.$ equiv) in dry $\mathrm{CH}_{2} \mathrm{Cl}_{2}(0.5 \mathrm{~mL}, 0.1 \mathrm{M})$ was added $\mathrm{Et}_{3} \mathrm{~N}(10 \mu \mathrm{L}, 0.06 \mathrm{mmol}, 1.2$ equiv) outside of the glovebox. The mixture was stirred at room temperature for 1

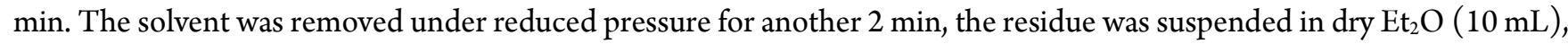
and the suspension was filtered through Celite to remove the insoluble triethylammonium tetrafluoroborate. The filtrate was concentrated in vacuo. The allenyliron complex $1 \mathrm{a}$ was dissolved in $2.5 \mathrm{~mL} \mathrm{C}_{6} \mathrm{D}_{6}$ in the glovebox. Into two of the NMR tubes were added $0.5 \mathrm{~mL}$ of the stock solution, and into another NMR tube charged with 9,10-dihydroanthracene (DHA) $\left(0.9 \mathrm{mg}, 5.0 \times 10^{-3} \mathrm{mmol}, 50 \%\right)$ was added $0.5 \mathrm{~mL}$ of the stock solution. The three NMR tubes were moved out of the glovebox, and into one of the NMR tubes containing $0.5 \mathrm{~mL}$ stock solution was added 2,2,6,6-Tetramethyl-1piperidinyloxy (TEMPO) ( $3 \mu \mathrm{L}$ of $0.15 \mathrm{M}$ stock solution, $5 \%$ ). Then the NMR tubes were placed close to each other into an oil bath preheated at $45.0^{\circ} \mathrm{C}$ for $60 \mathrm{~min}$. Then the NMR tube was taken out of the oil bath for subsequent ${ }^{1} \mathrm{H}-\mathrm{NMR}$ spectroscopy.

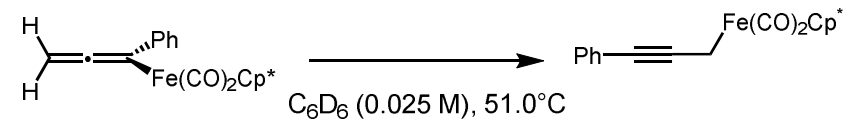

$85 \%$ conversion

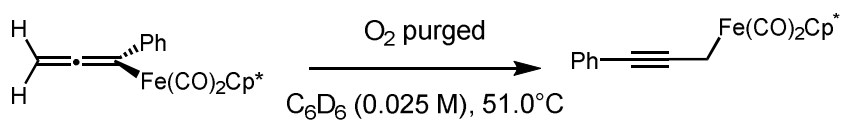

$64 \%$ conversion

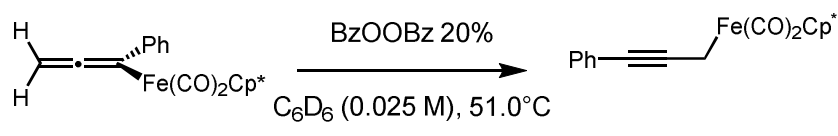

$65 \%$ conversion

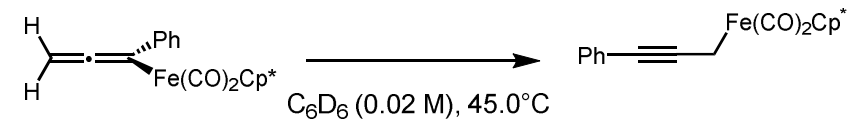

$60 \%$ conversion
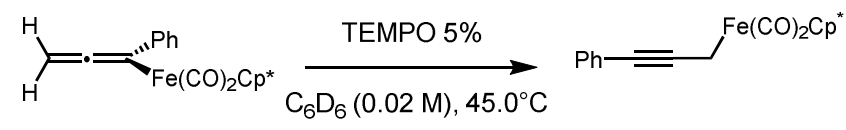

$7 \%$ conversion
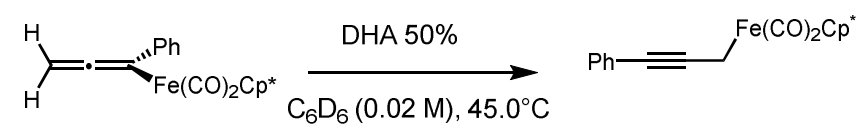

$44 \%$ conversion

Scheme S3: Radical scavengers' effect on the $\eta^{1}$-allenyliron to $\eta^{1}$-propargyliron isomerization

\subsection{Effect of added silver iodide or silver(0)}

To a solution of $\eta^{2}$-1-Phenyl-1-propyne pentamethylcyclopentadienyliron dicarbonyl tetrafluoroborate complexes 1-I (23.0 mg, $0.05 \mathrm{mmol}, 1.0$ equiv) in dry $\mathrm{CH}_{2} \mathrm{Cl}_{2}(0.5 \mathrm{~mL}, 0.1 \mathrm{M})$ was added $\mathrm{Et}_{3} \mathrm{~N}$ (10 $\mu \mathrm{L}, 0.06 \mathrm{mmol}, 1.2$ equiv) outside of the glovebox. The mixture was stirred at room temperature for $1 \mathrm{~min}$. The solvent was removed under reduced pressure

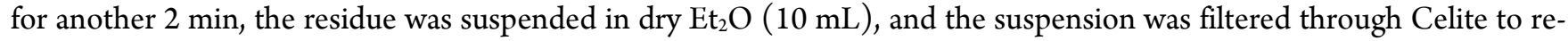
move the insoluble triethylammonium tetrafluoroborate. The filtrate was concentrated in vacuo. The allenyliron complex 1 a was dissolved in $1.0 \mathrm{~mL} \mathrm{C}_{6} \mathrm{D}_{6}$ in the glovebox. Into one NMR tube charged with freshly prepared AgI $(0.2 \mathrm{mg}, 5 \%)$ was added $0.5 \mathrm{~mL}$ of the stock solution, and into another NMR tubes charged with $\mathrm{Ag}(0.5 \mathrm{mg}, 20 \%)$ were added $0.5 \mathrm{~mL}$ of the stock solution. The two NMR tubes were kept in room temperature for $30 \mathrm{~min}$ for subsequent ${ }^{1} \mathrm{H}$-NMR spectroscopy. 


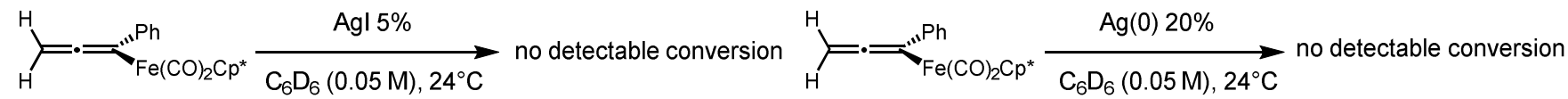

Scheme S4: Effect of added silver source on the $\eta^{1}$-allenyliron to $\eta^{1}$-propargyliron isomerization 


\section{X-Ray structures for products}
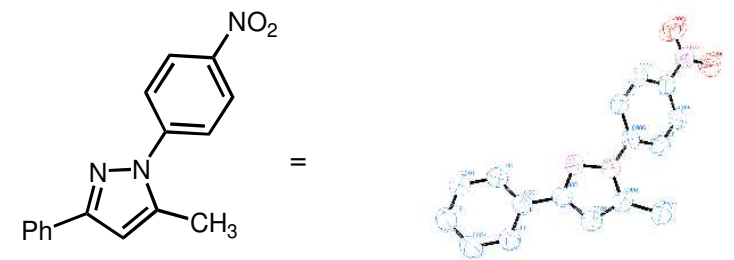

Figure S10: ORTEP for 10c (CCDC 2054882)

\section{Datablock: jz05921_0ma_a}

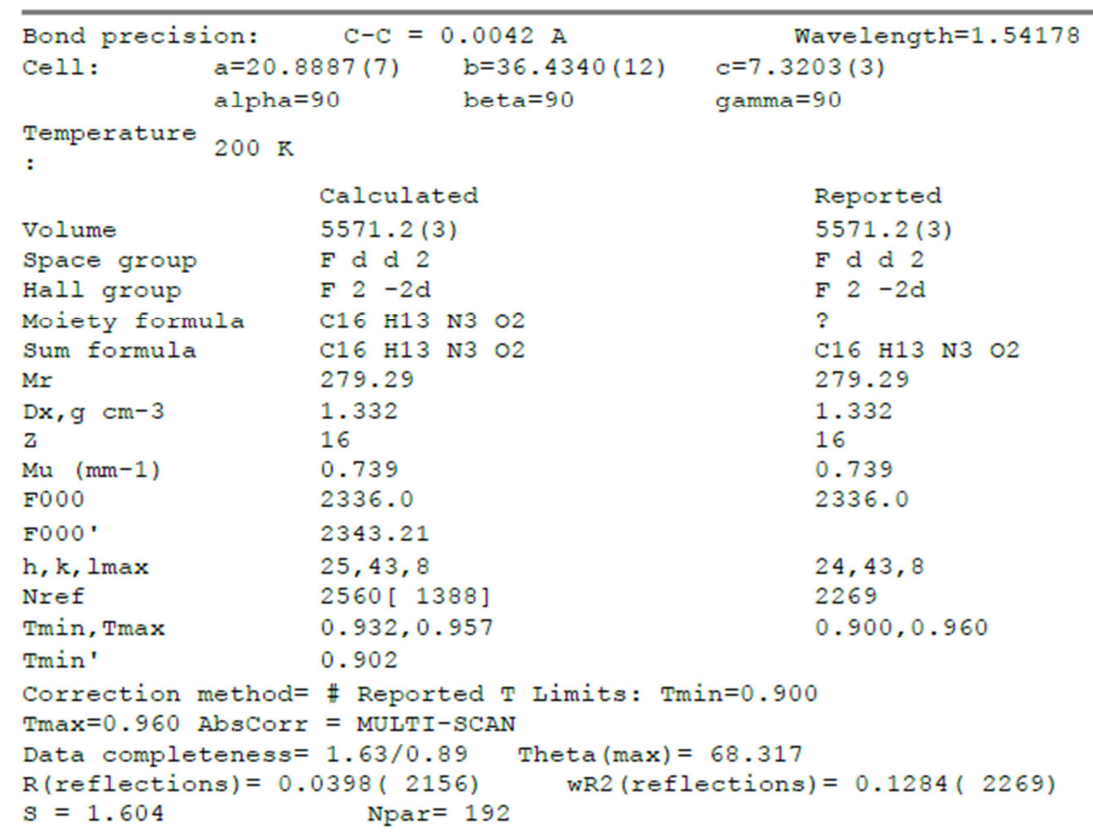




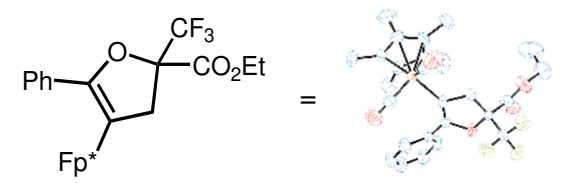

Figure S1 1: ORTEP for 3 (CCDC 2059267)

\section{Datablock: JZ051323_a}

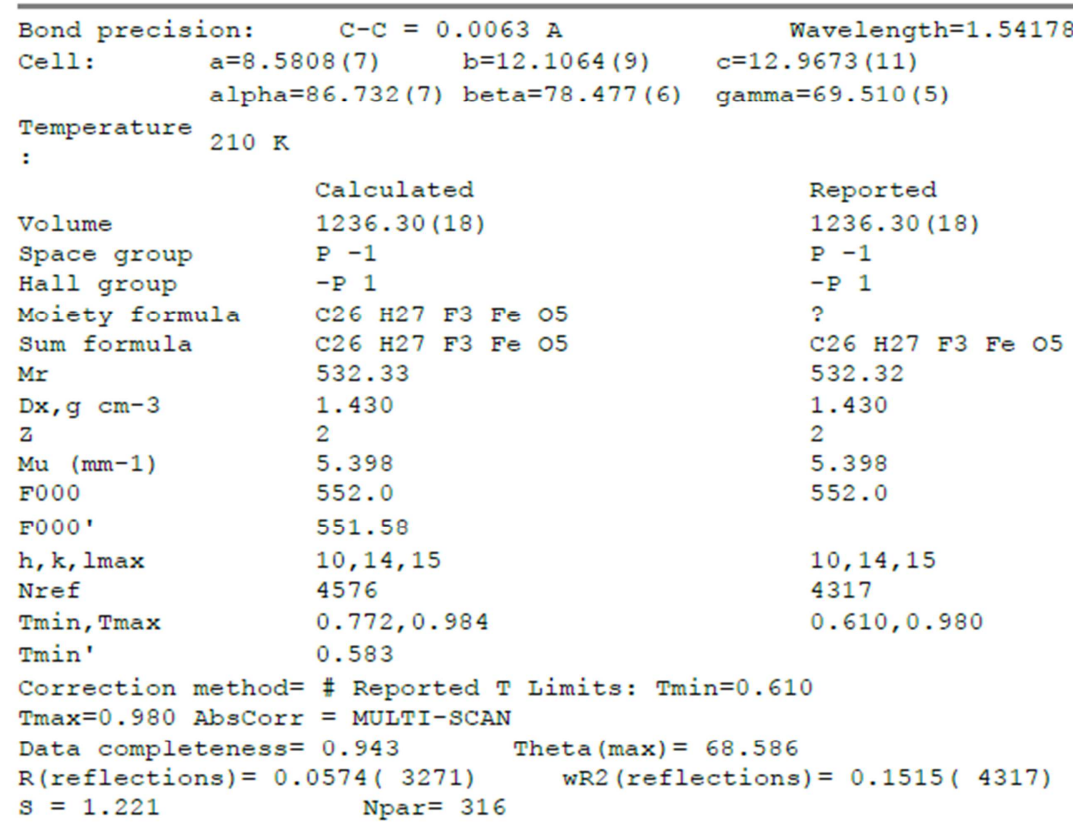




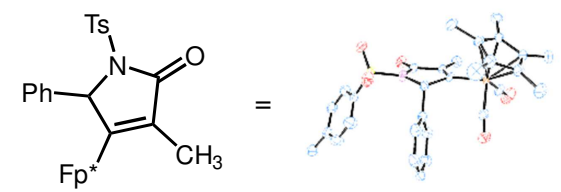

Figure S12: ORTEP for 6 (CCDC 2059006)

\section{Datablock: Corcoran2_b}

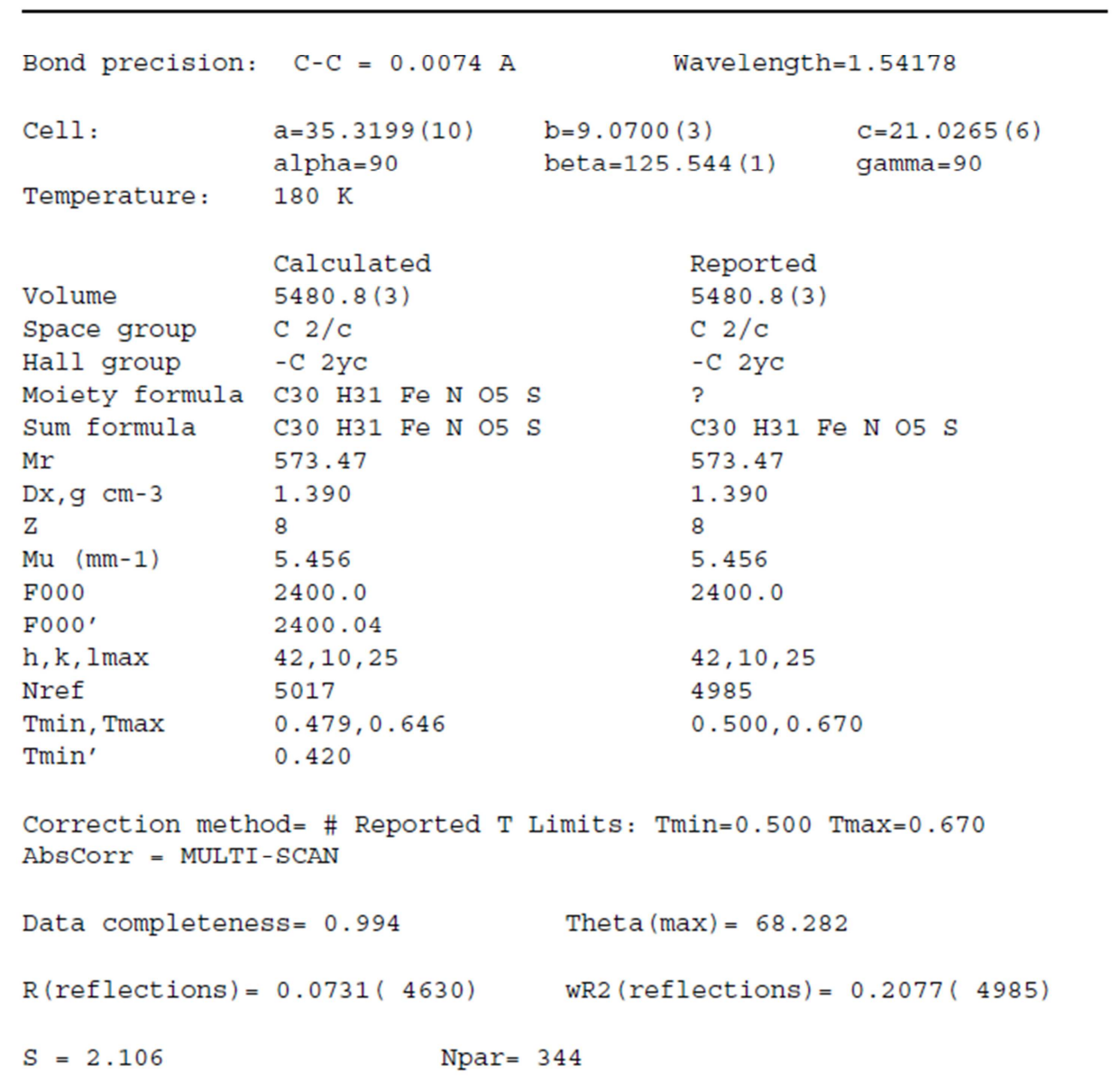




\section{Copies of NMR spectra}

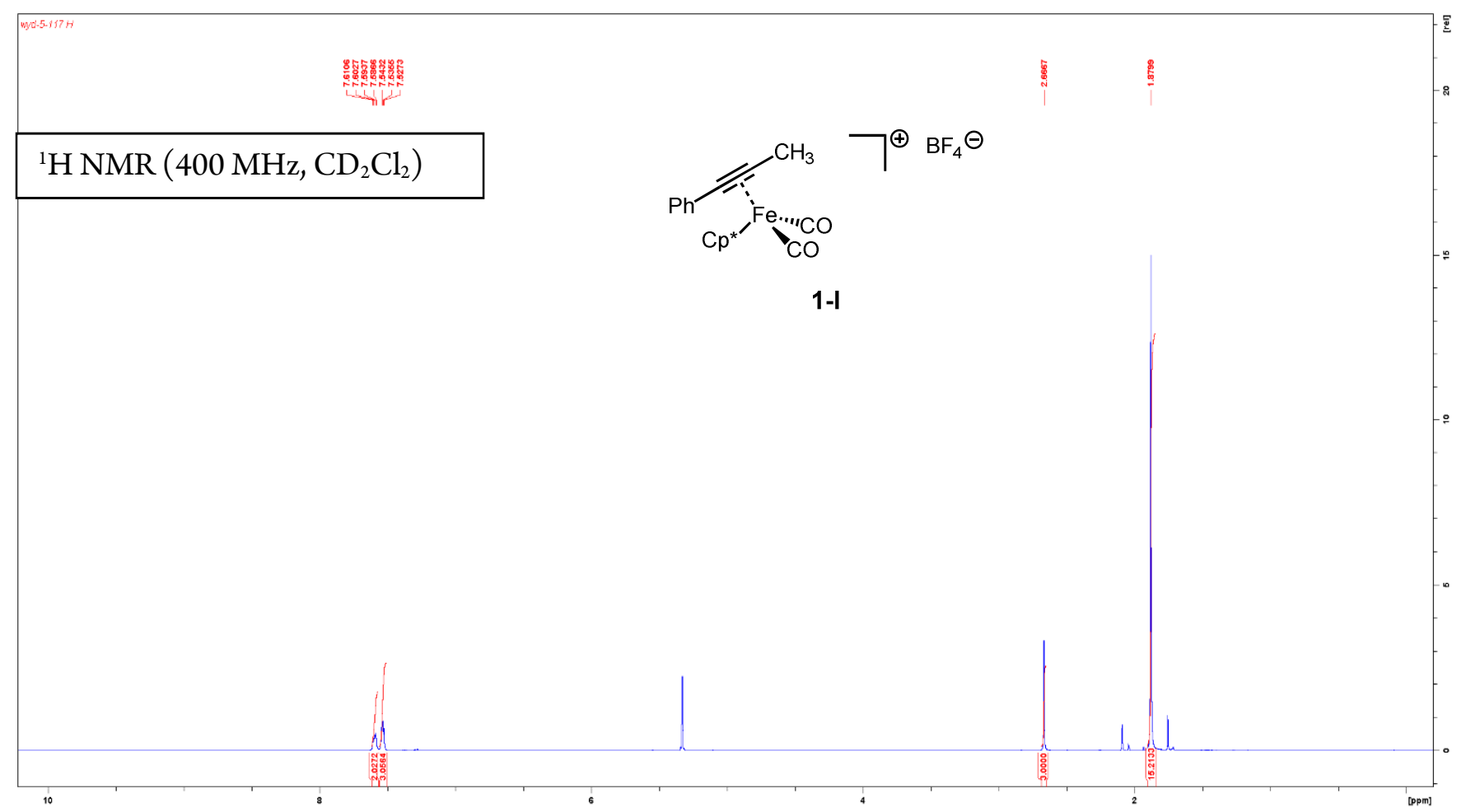

Figure S13: ${ }^{1} \mathrm{H}$ NMR spectrum of 1-I

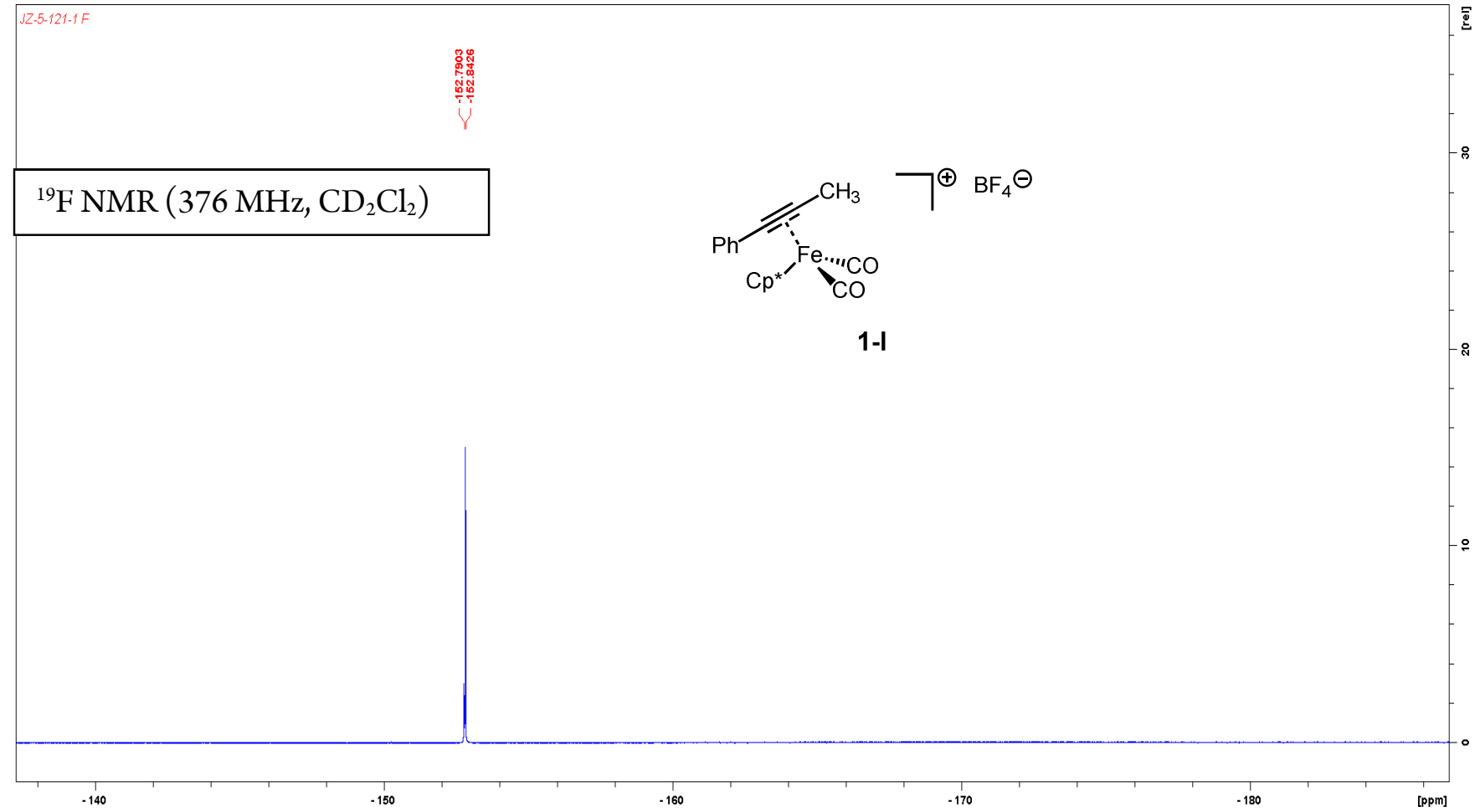

Figure S14: ${ }^{19} \mathrm{~F}$ NMR spectrum of 1-I 


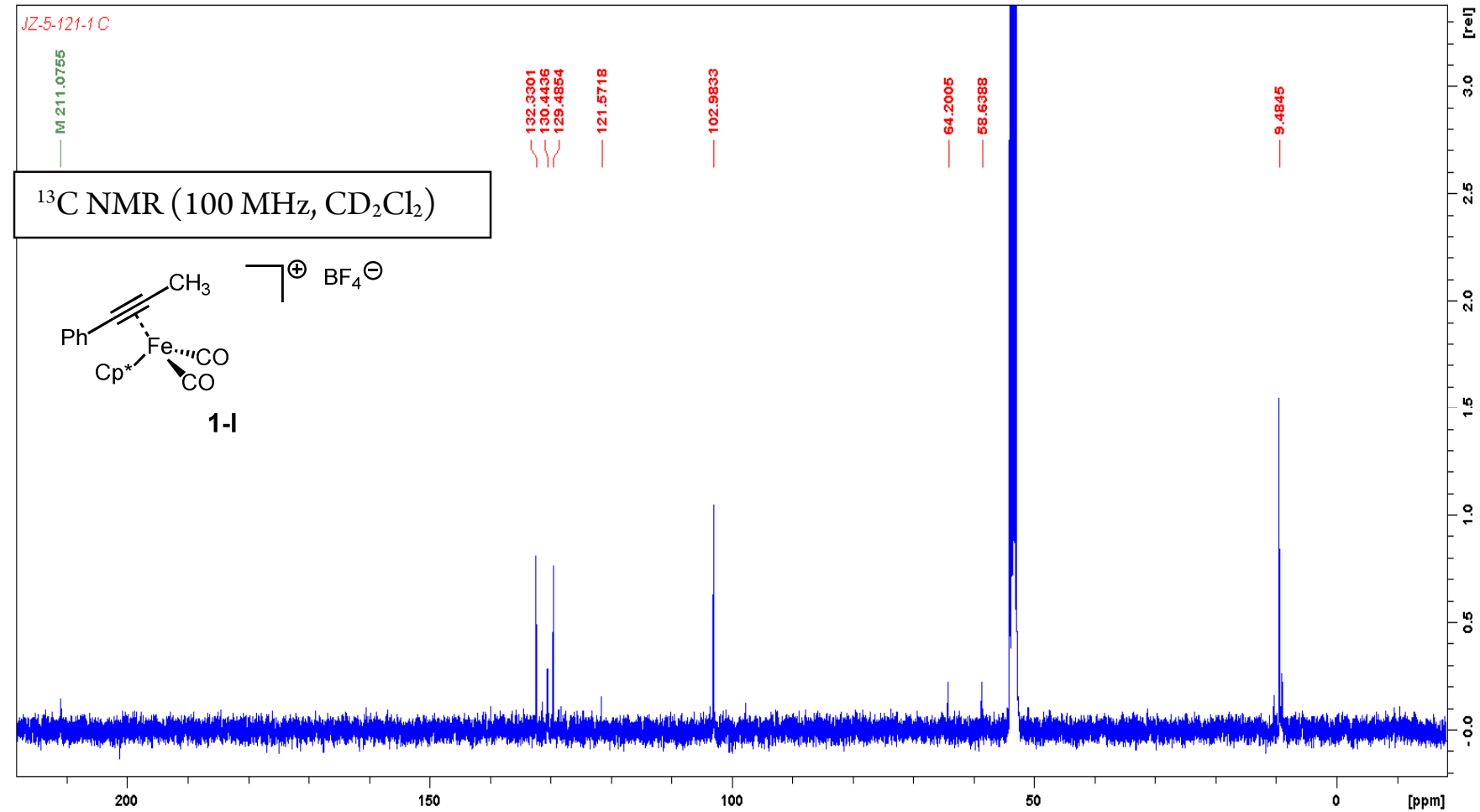

Figure S15: ${ }^{13} \mathrm{C}$ NMR spectrum of 1-I 


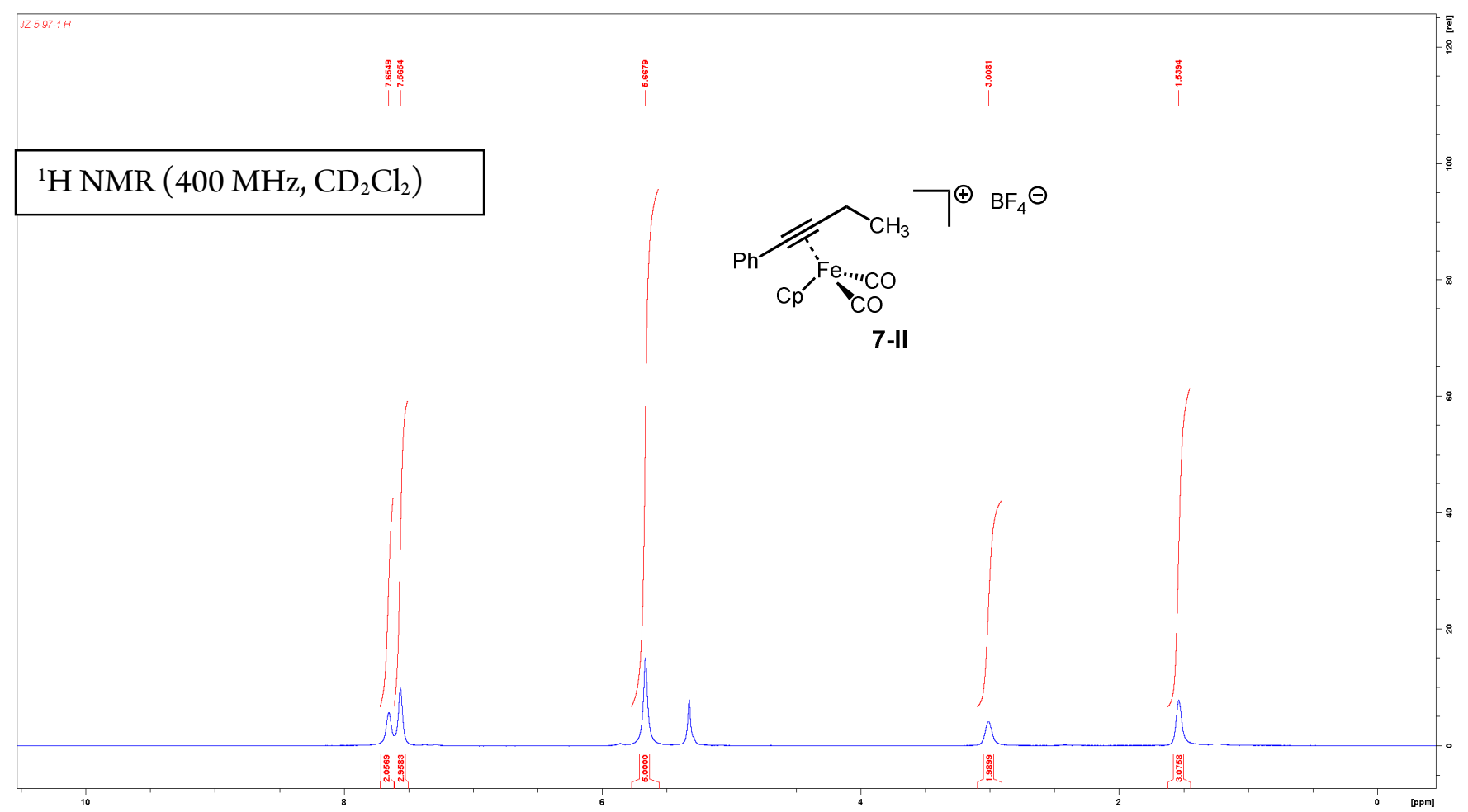

Figure S16: ${ }^{1} \mathrm{H}$ NMR spectrum of 7-II

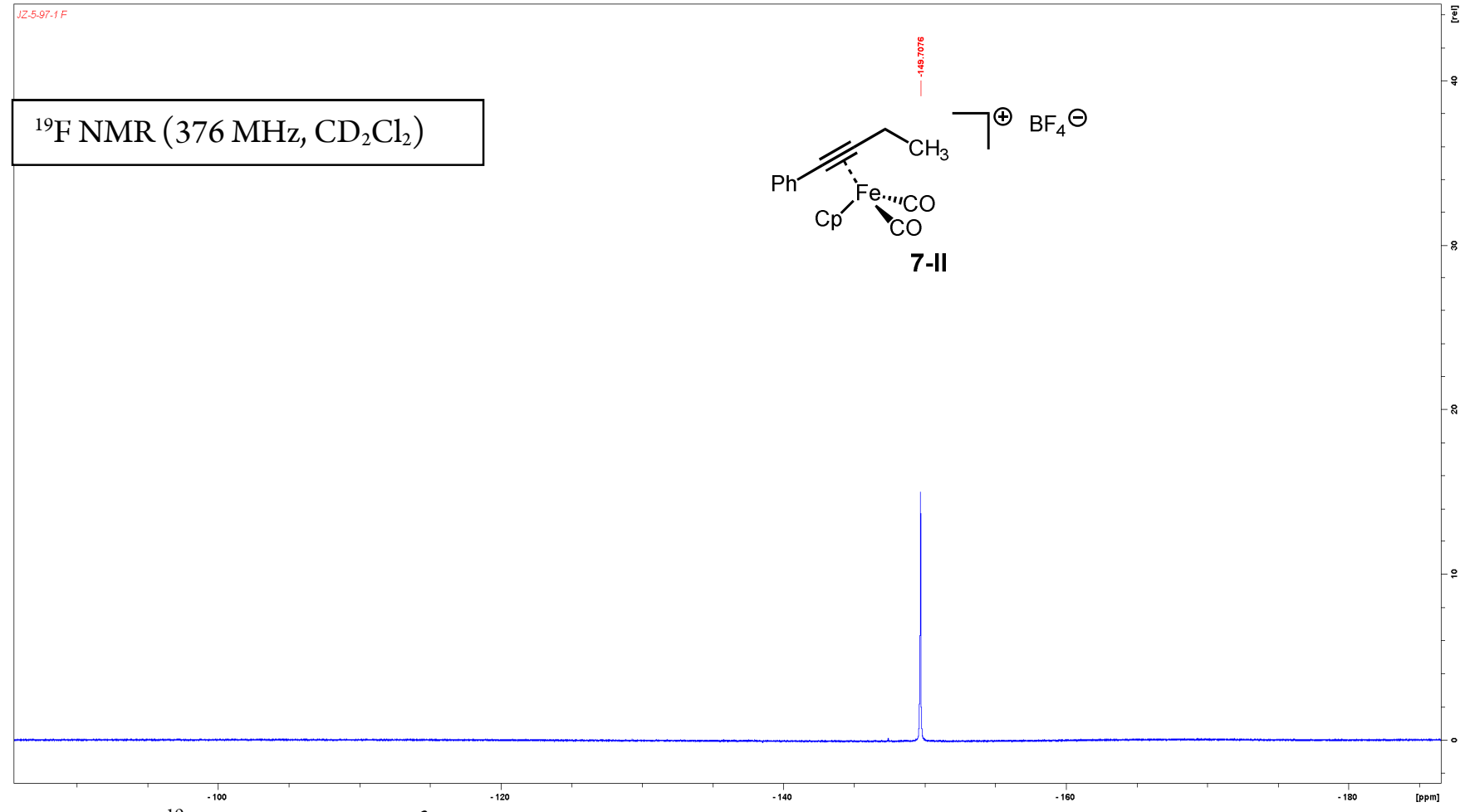

Figure S17: ${ }^{19} \mathrm{~F}$ NMR spectrum of 7-II 


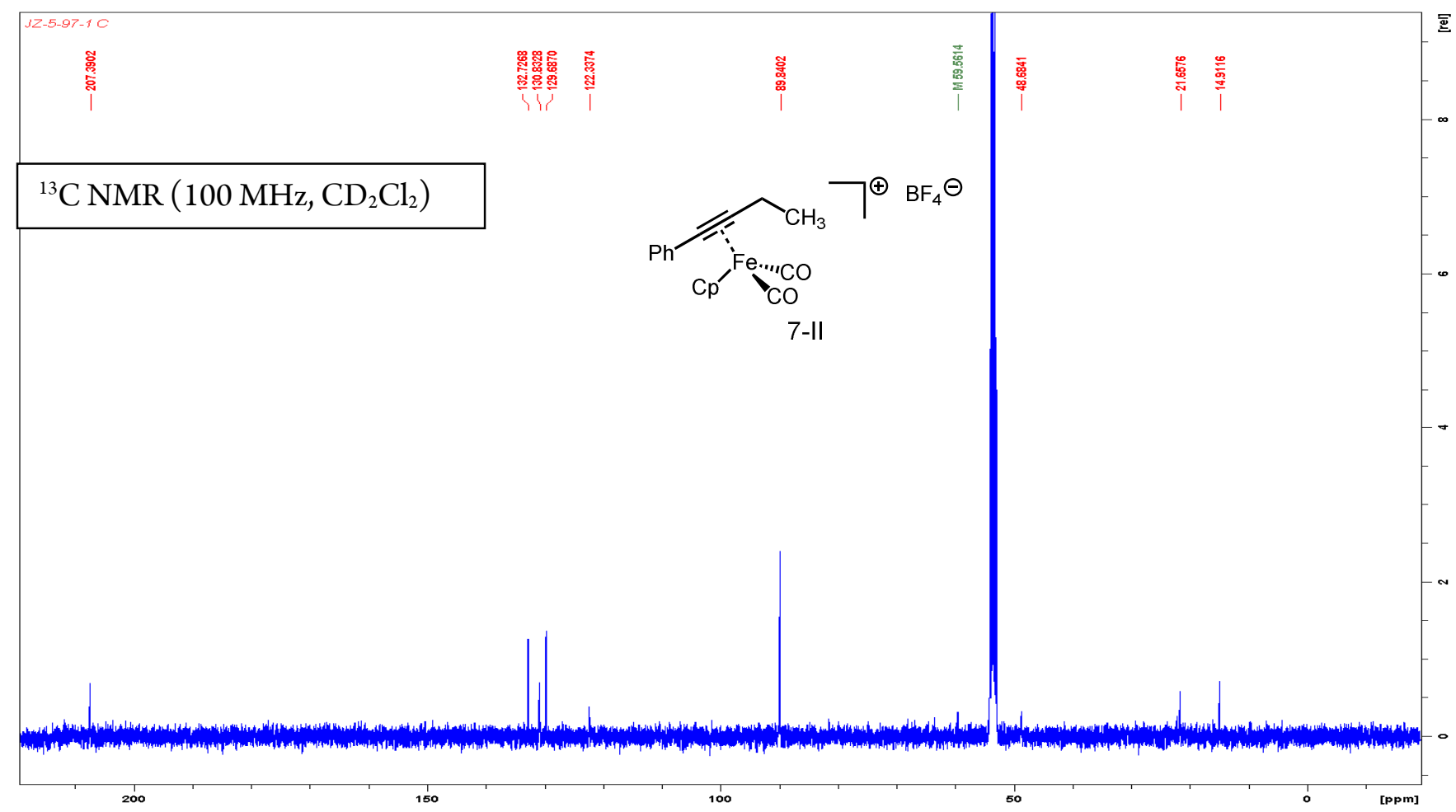

Figure S18: ${ }^{200} \mathrm{C}$ NMR spectrum of 7-II 


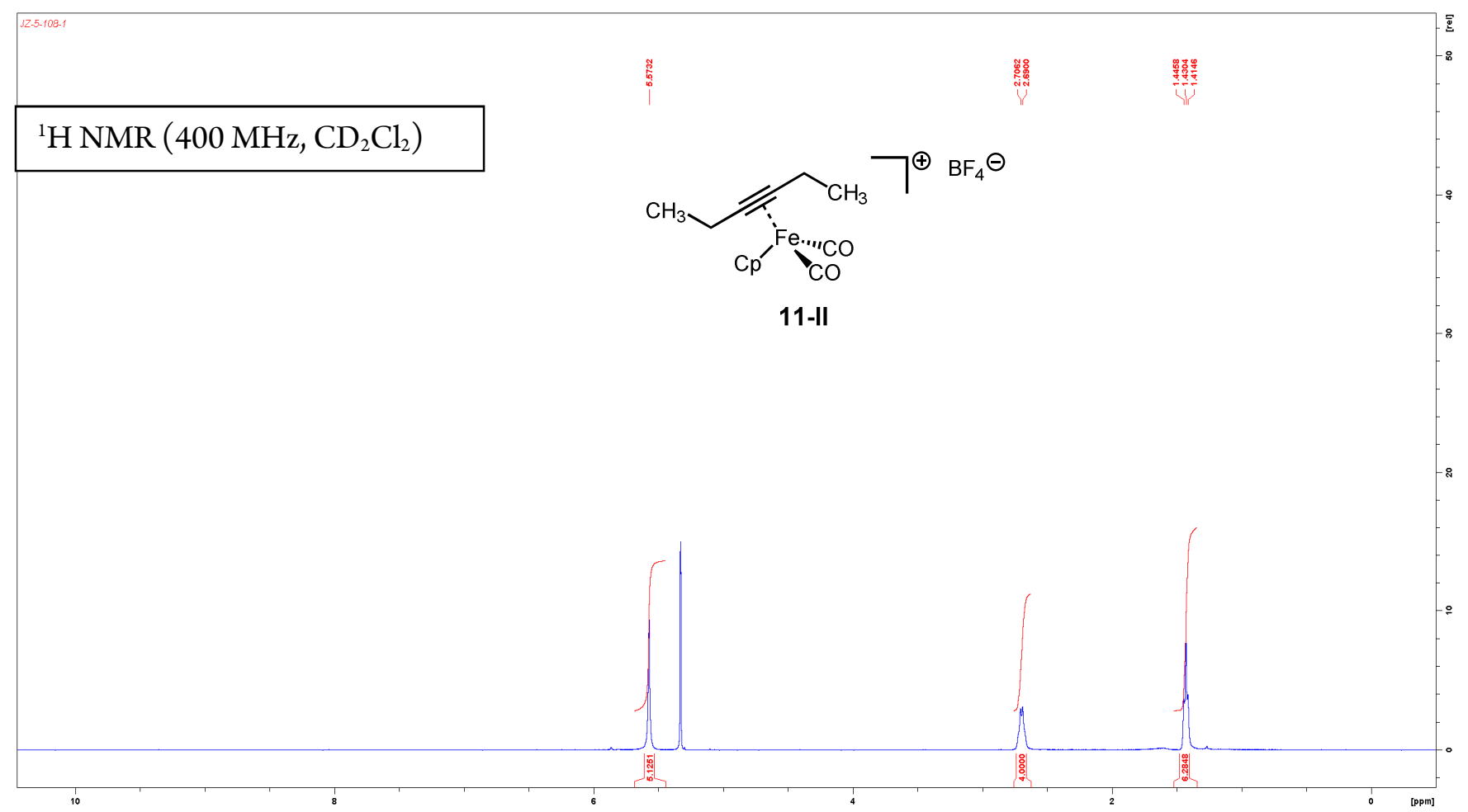

Figure S19: ${ }^{1} \mathrm{H}$ NMR spectrum of 11-II

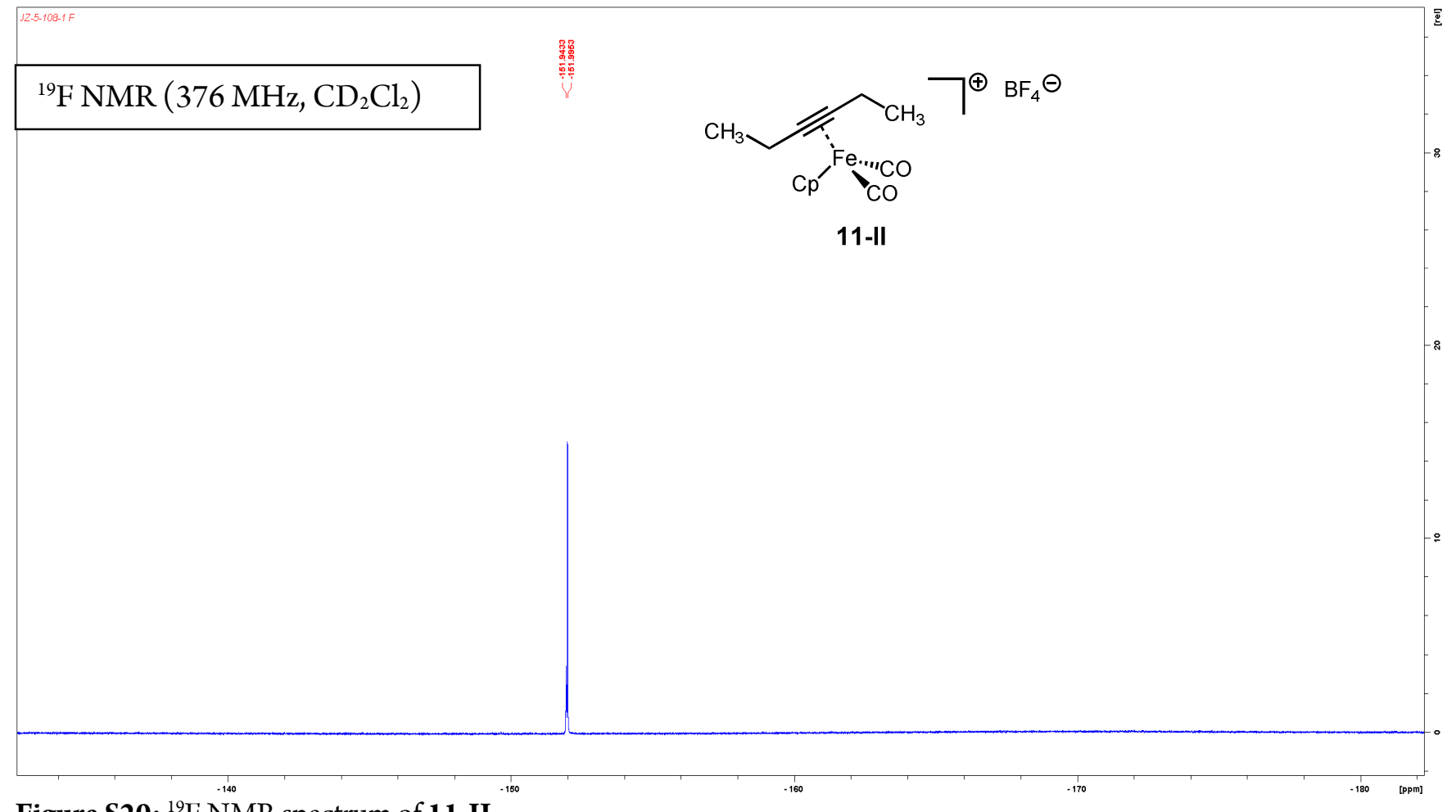

Figure S20: ${ }^{19} \mathrm{~F}$ NMR spectrum of 11-II 


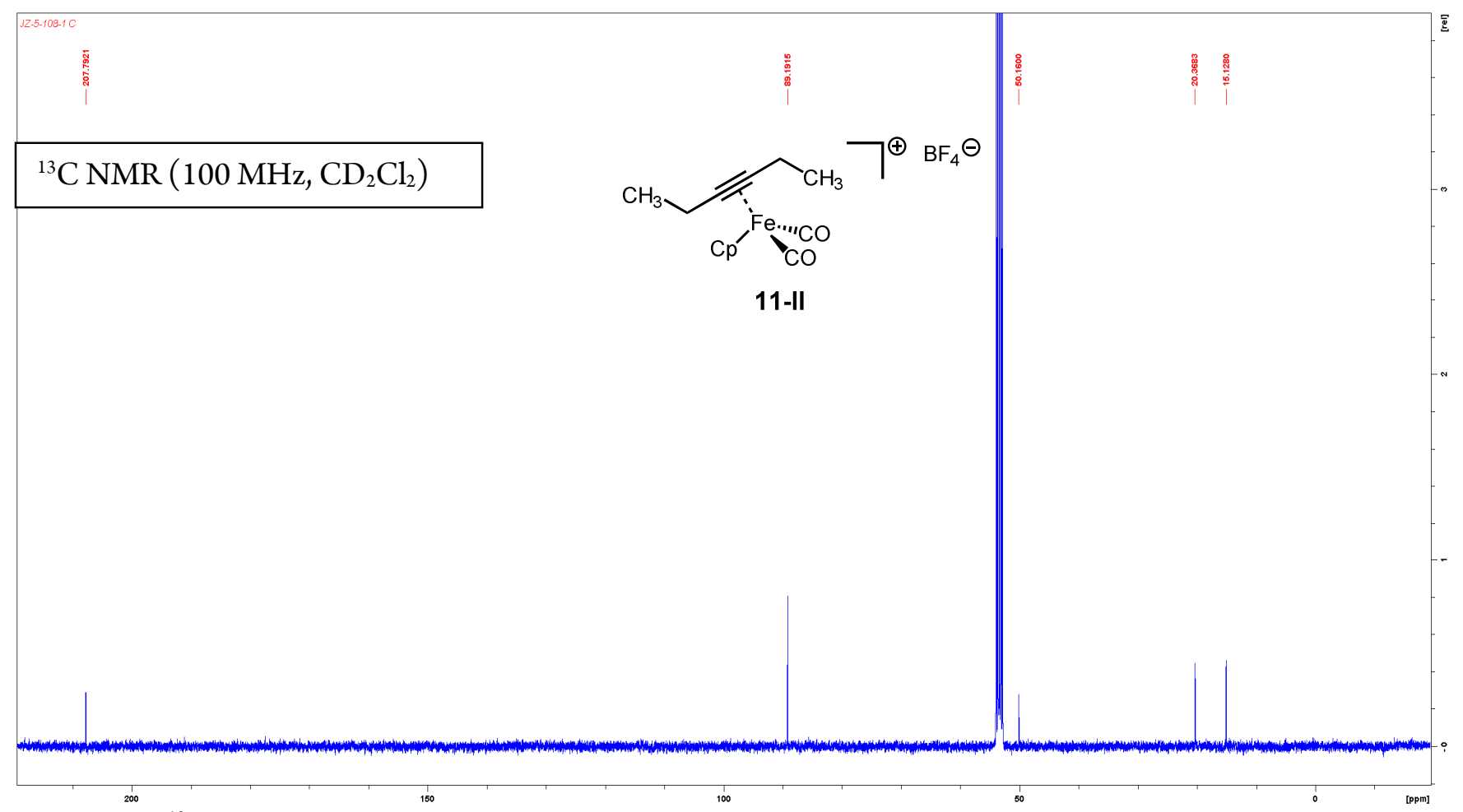

Figure S21: ${ }^{13} \mathrm{C}$ NMR spectrum of 11-II 


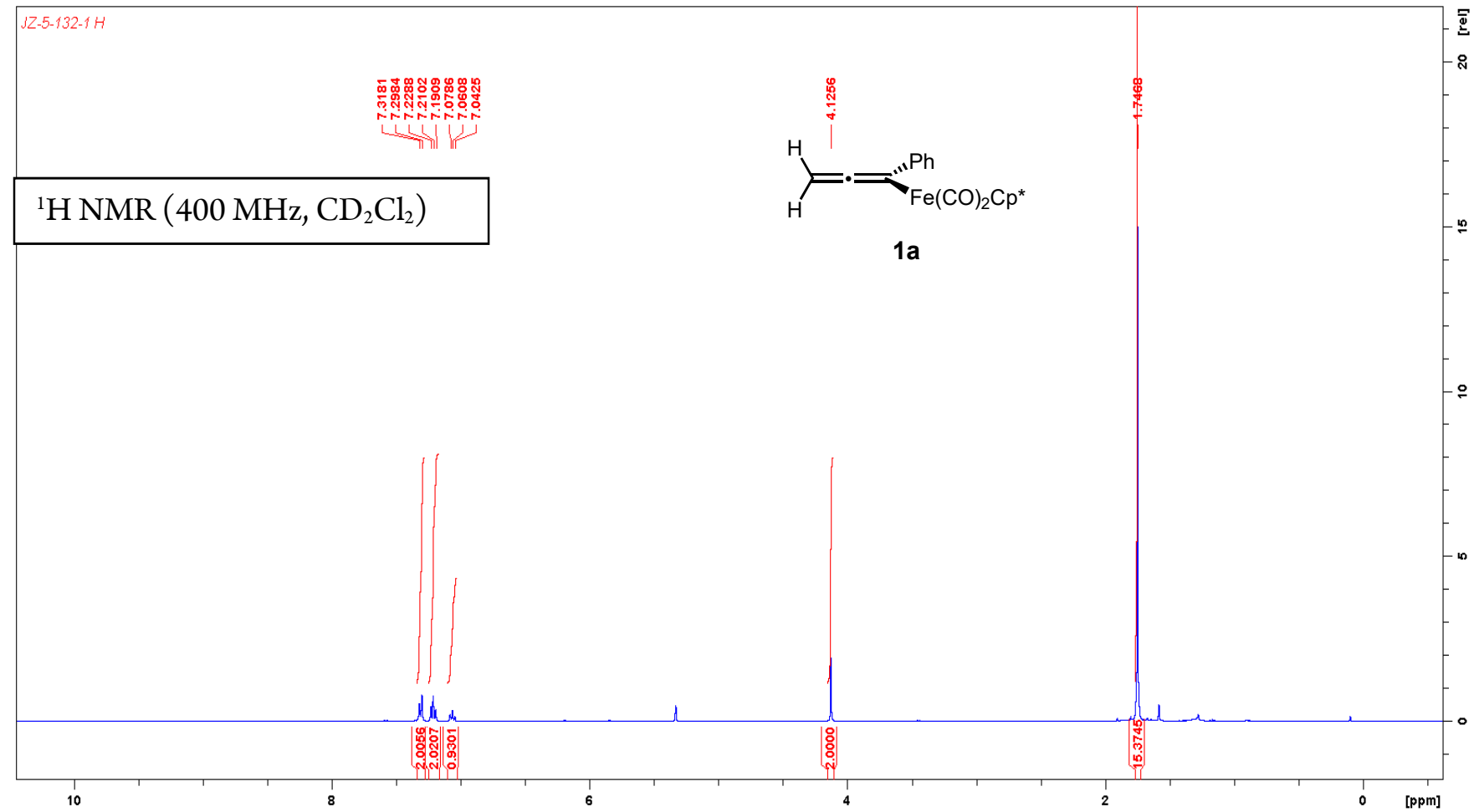

Figure S22: ${ }^{1} \mathrm{H}$ NMR spectrum of $\mathbf{1 a}$

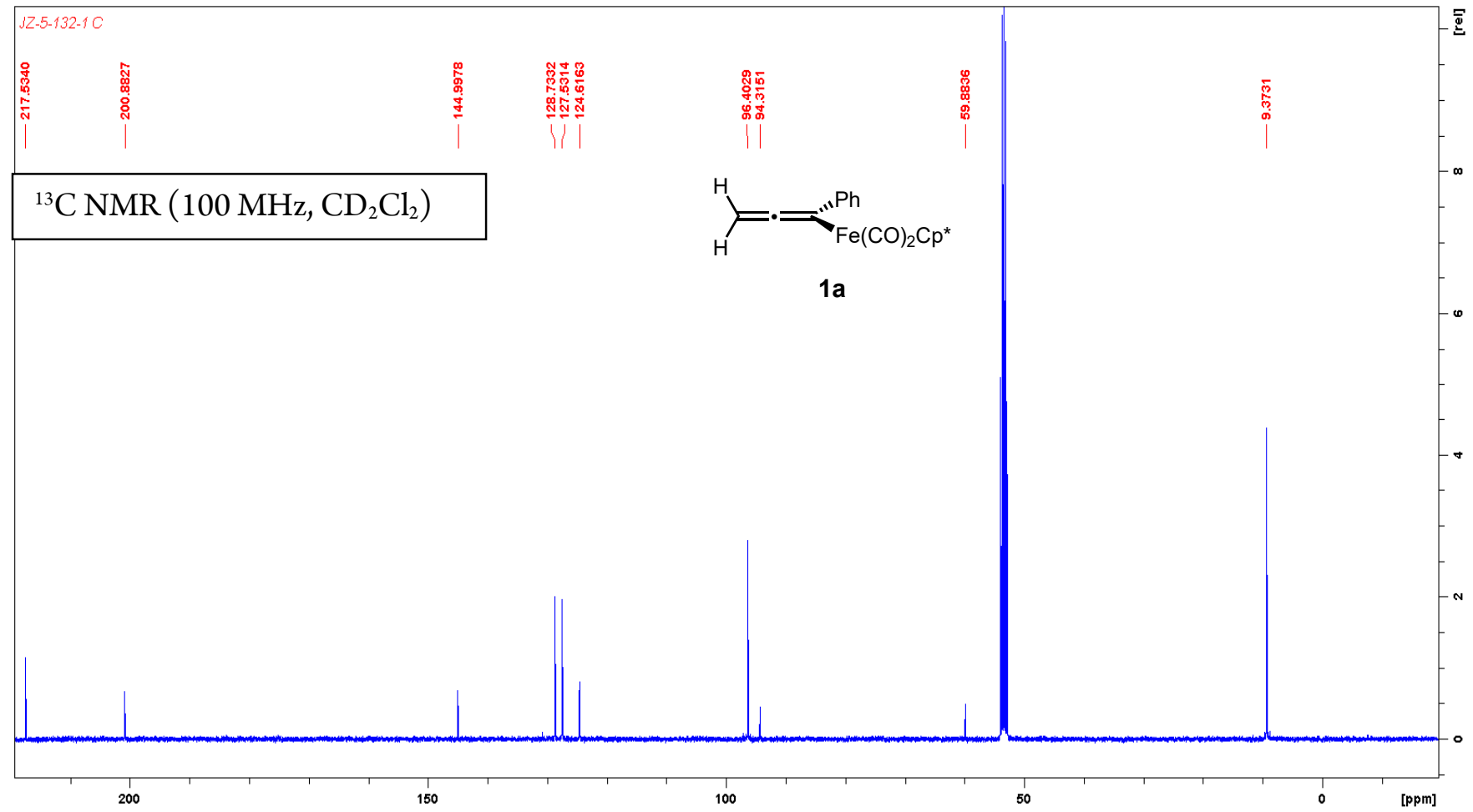

Figure S23: ${ }^{13} \mathrm{C}$ NMR spectrum of 1 a 


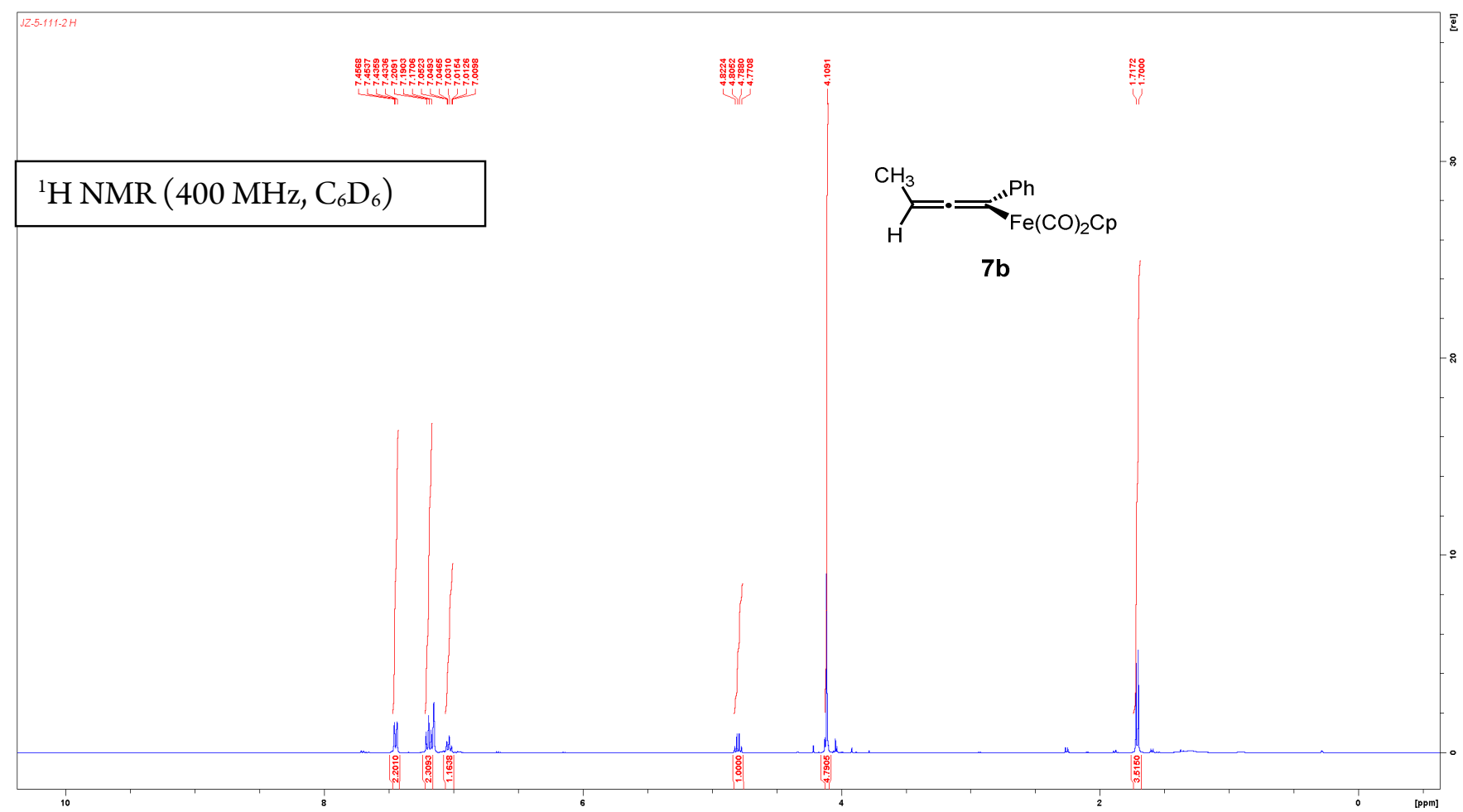

Figure S24: ${ }^{1} \mathrm{H}$ NMR spectrum of $\mathbf{7 b}$

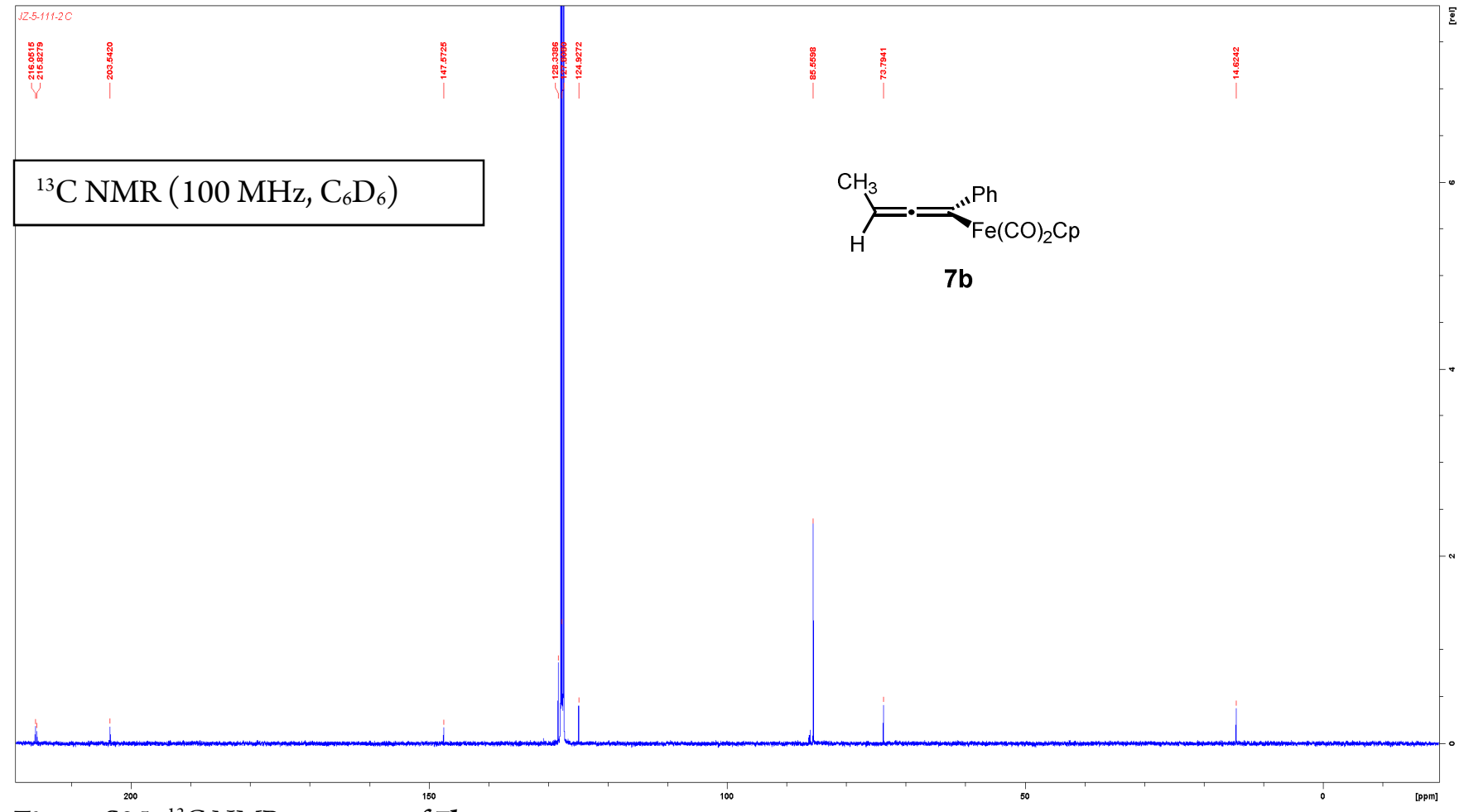

Figure S25: ${ }^{13} \mathrm{C}$ NMR spectrum of $7 \mathbf{b}$ 


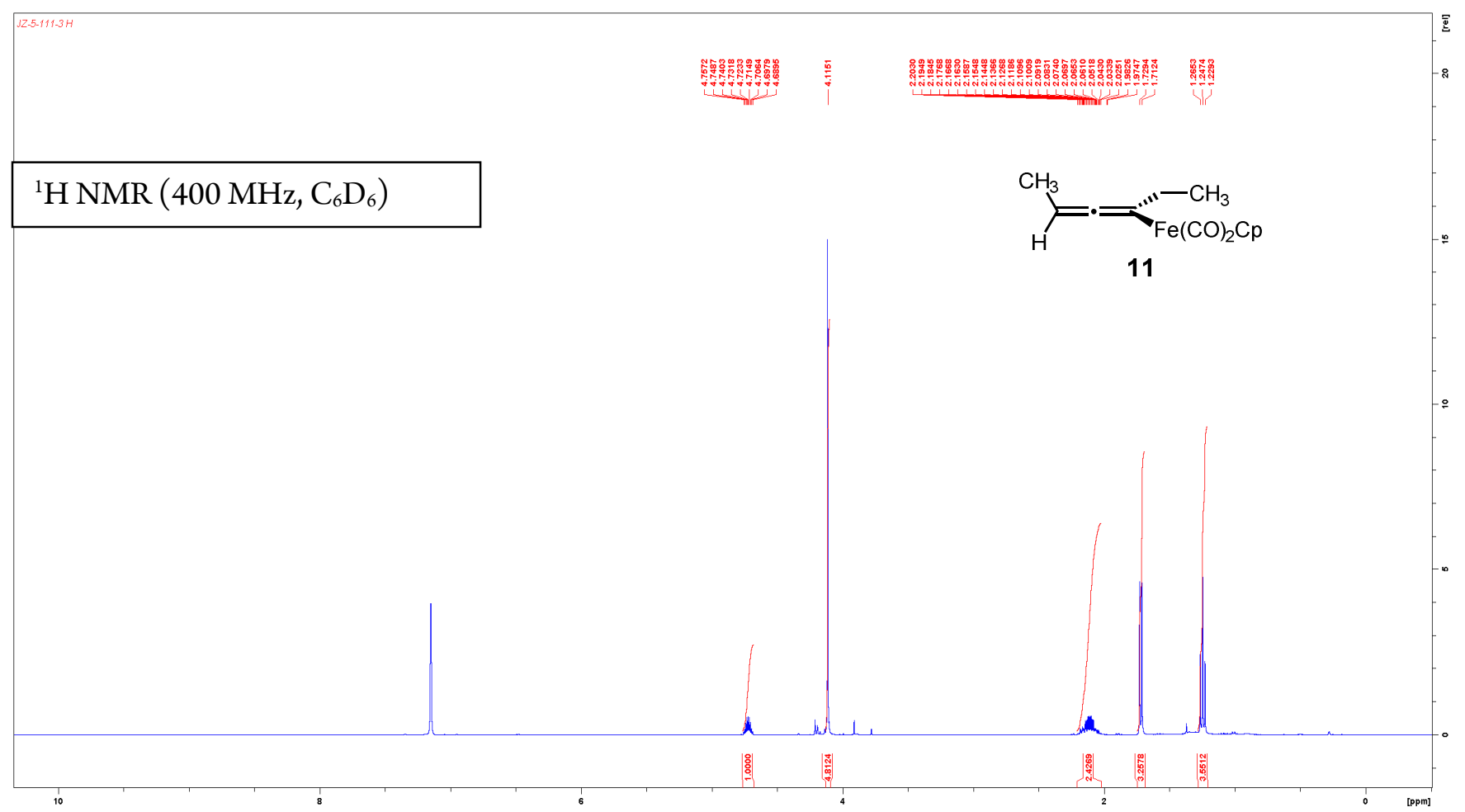

Figure S26: ${ }^{1} \mathrm{H}$ NMR spectrum of 11

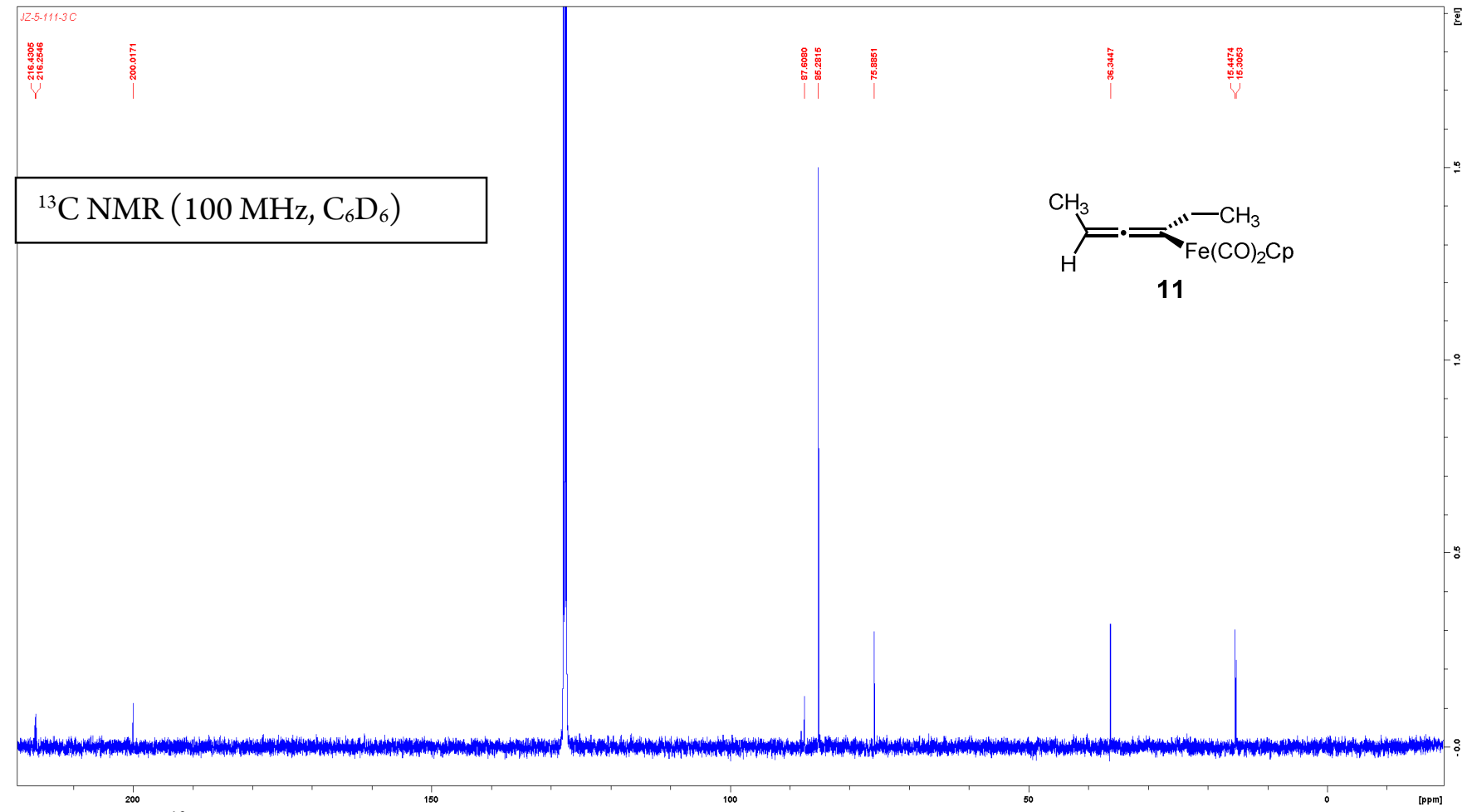

Figure S27: ${ }^{13} \mathrm{C}$ NMR spectrum of 11 


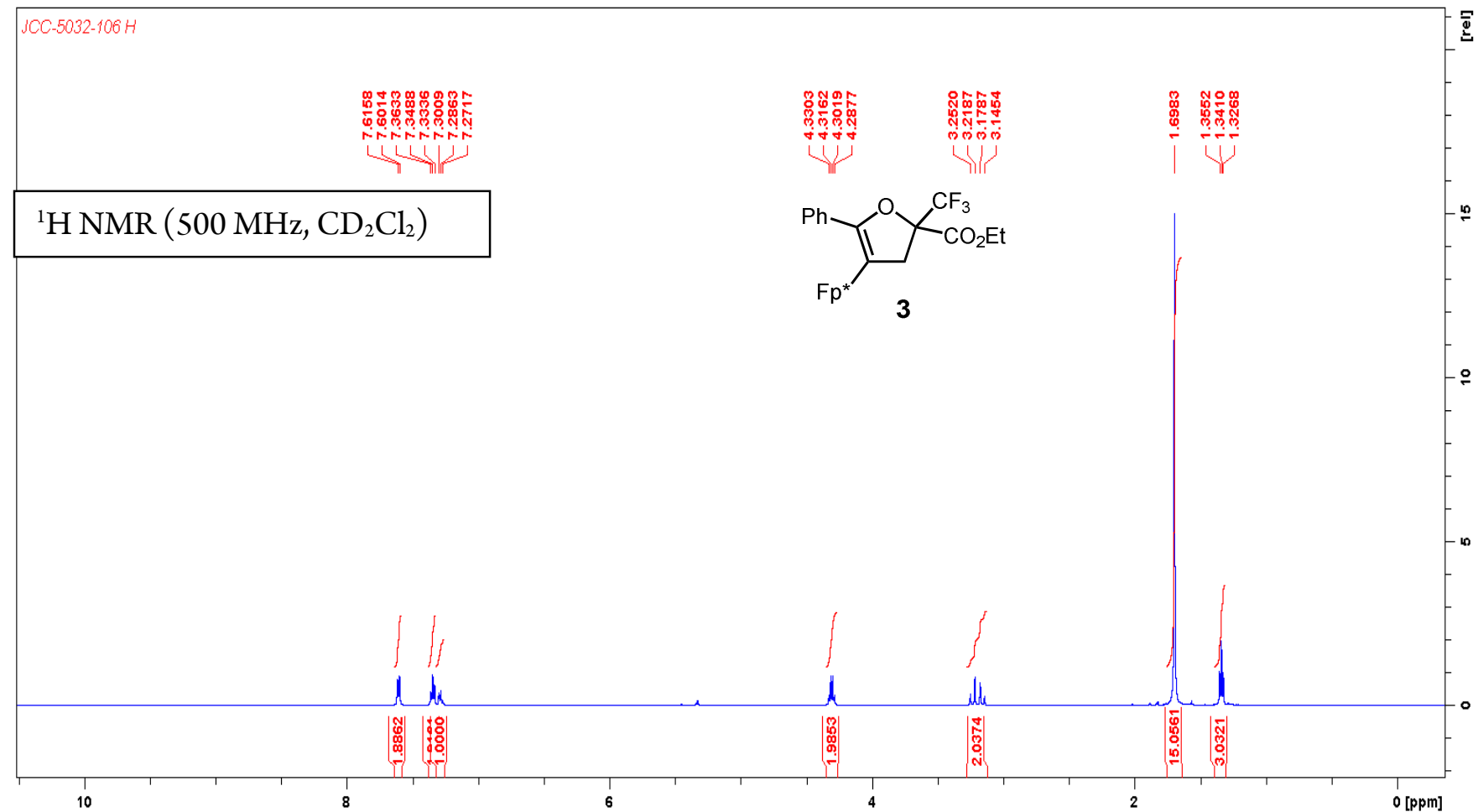

Figure S28: ${ }^{1} \mathrm{H}$ NMR spectrum of 3

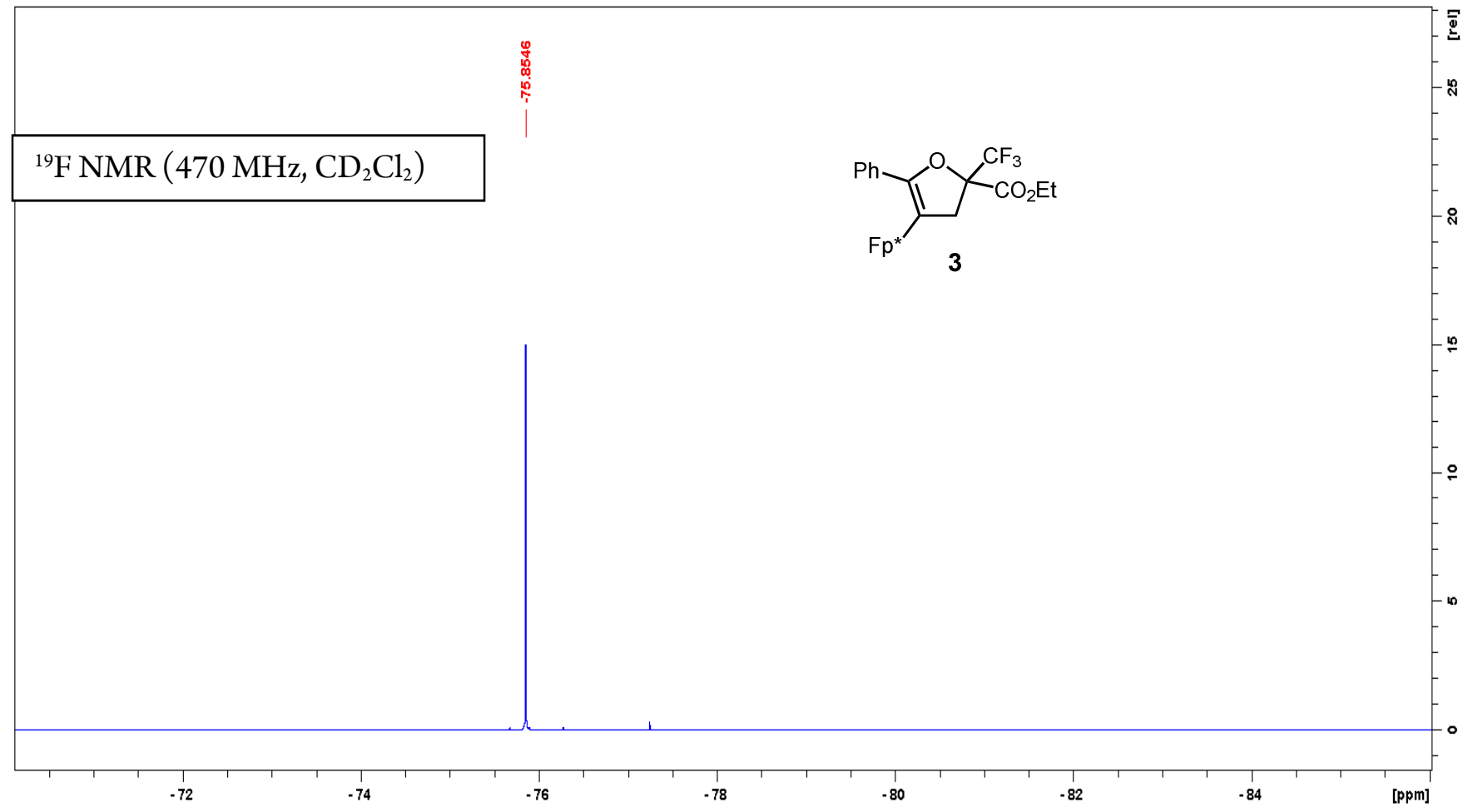

Figure S29: ${ }^{19} \mathrm{~F}$ NMR spectrum of 3 


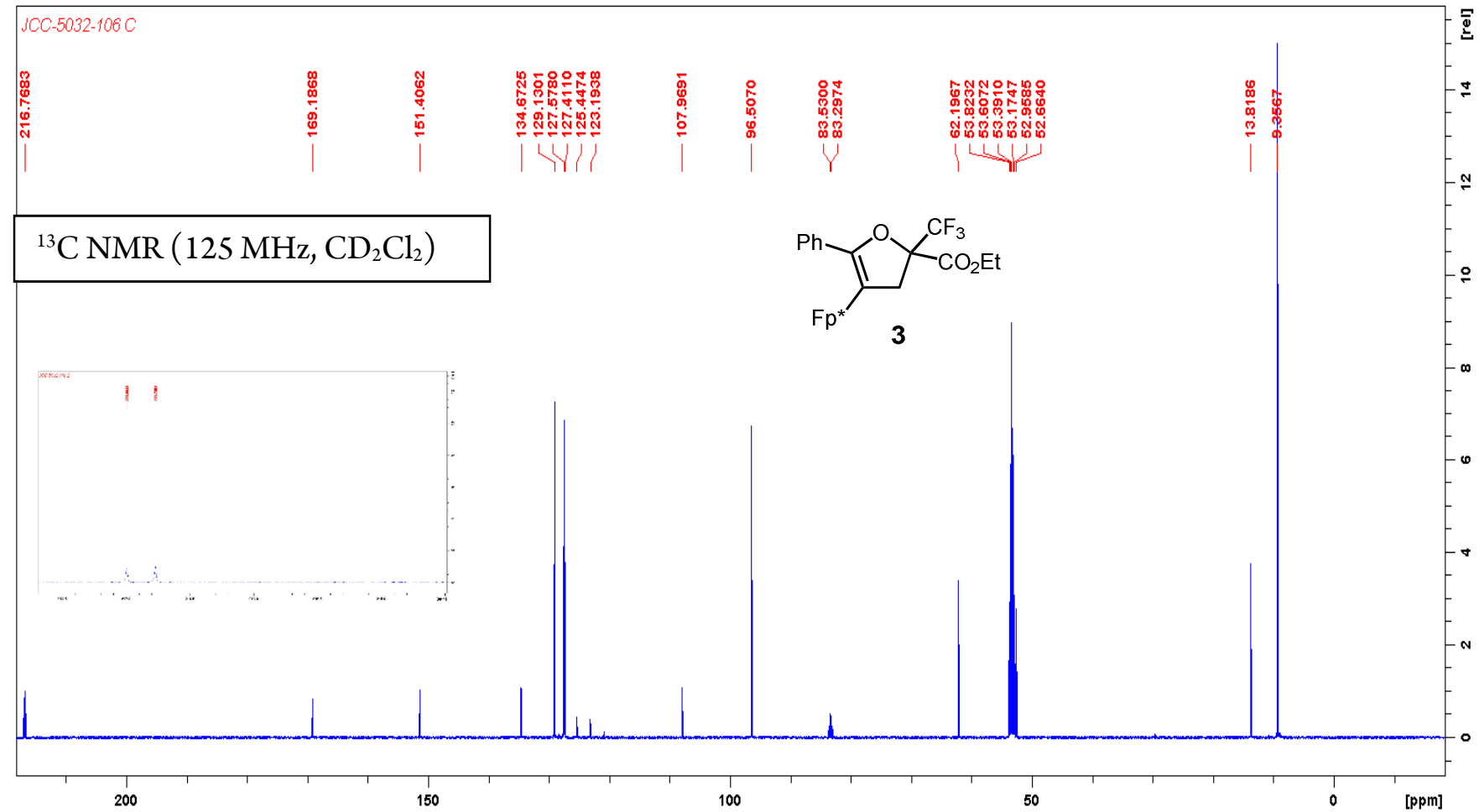

Figure S30: ${ }^{13} \mathrm{C}$ NMR spectrum of 3 


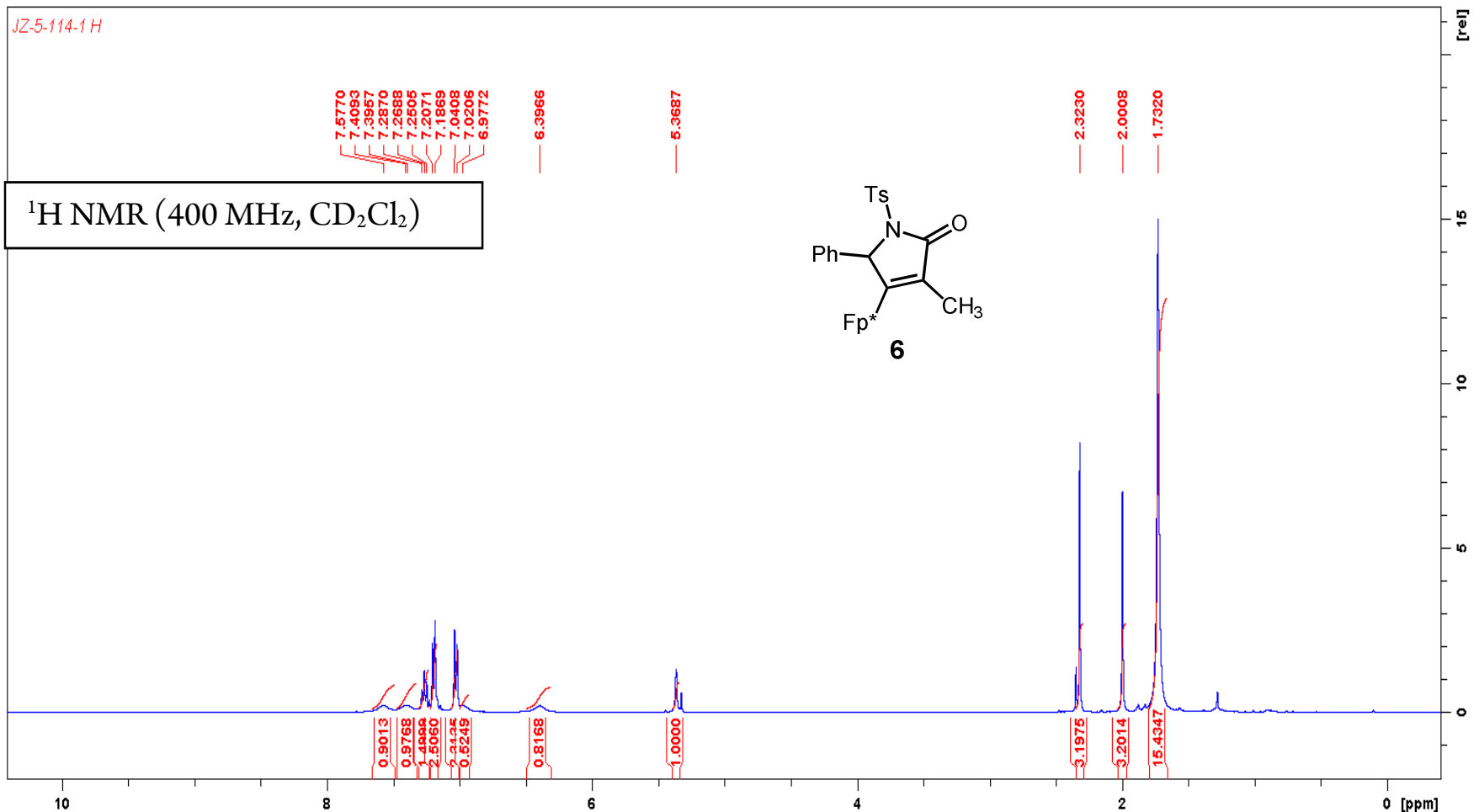

Figure S31: ${ }^{1} \mathrm{H}$ NMR spectrum of 6

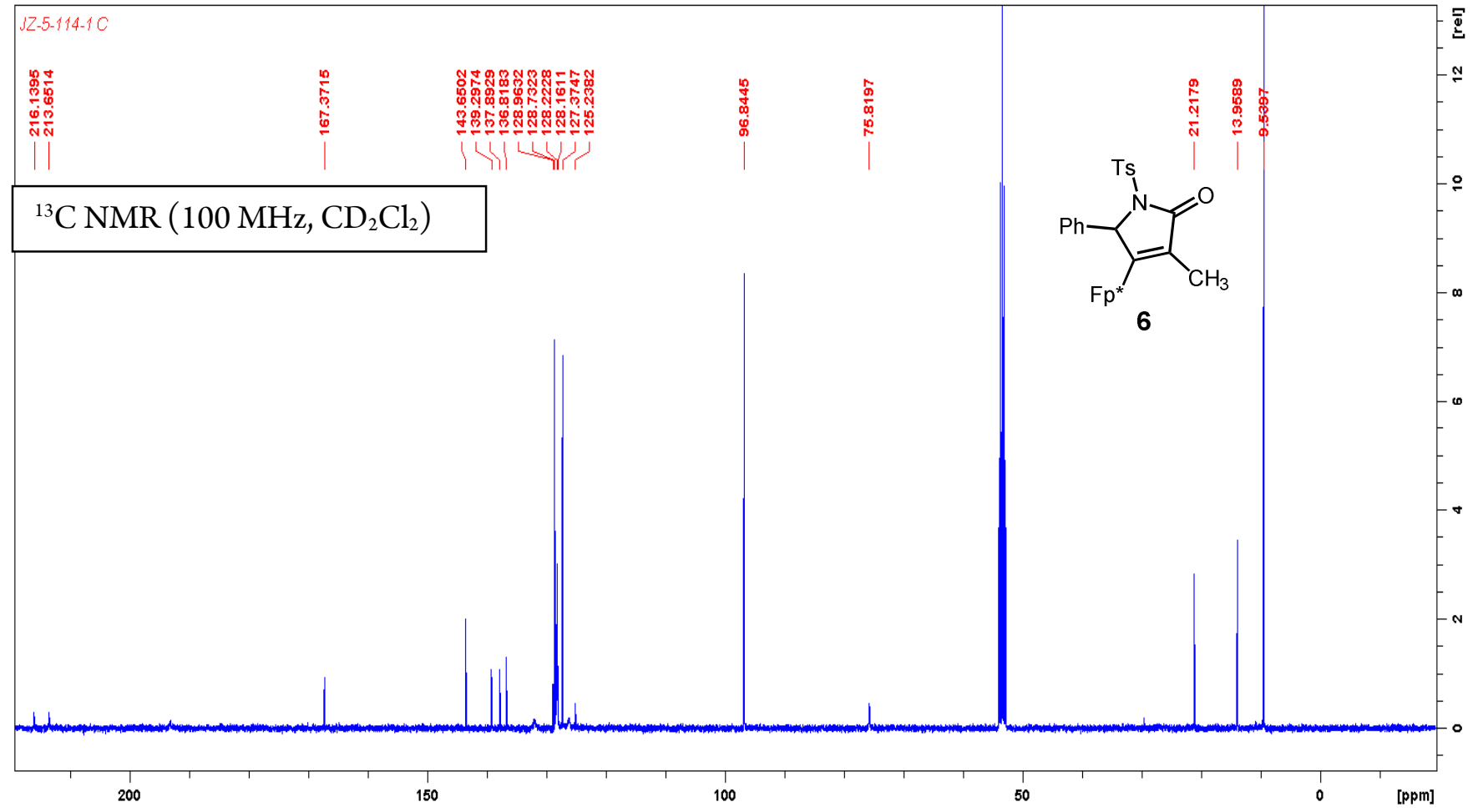

Figure S32: ${ }^{13} \mathrm{C}$ NMR spectrum of 6 


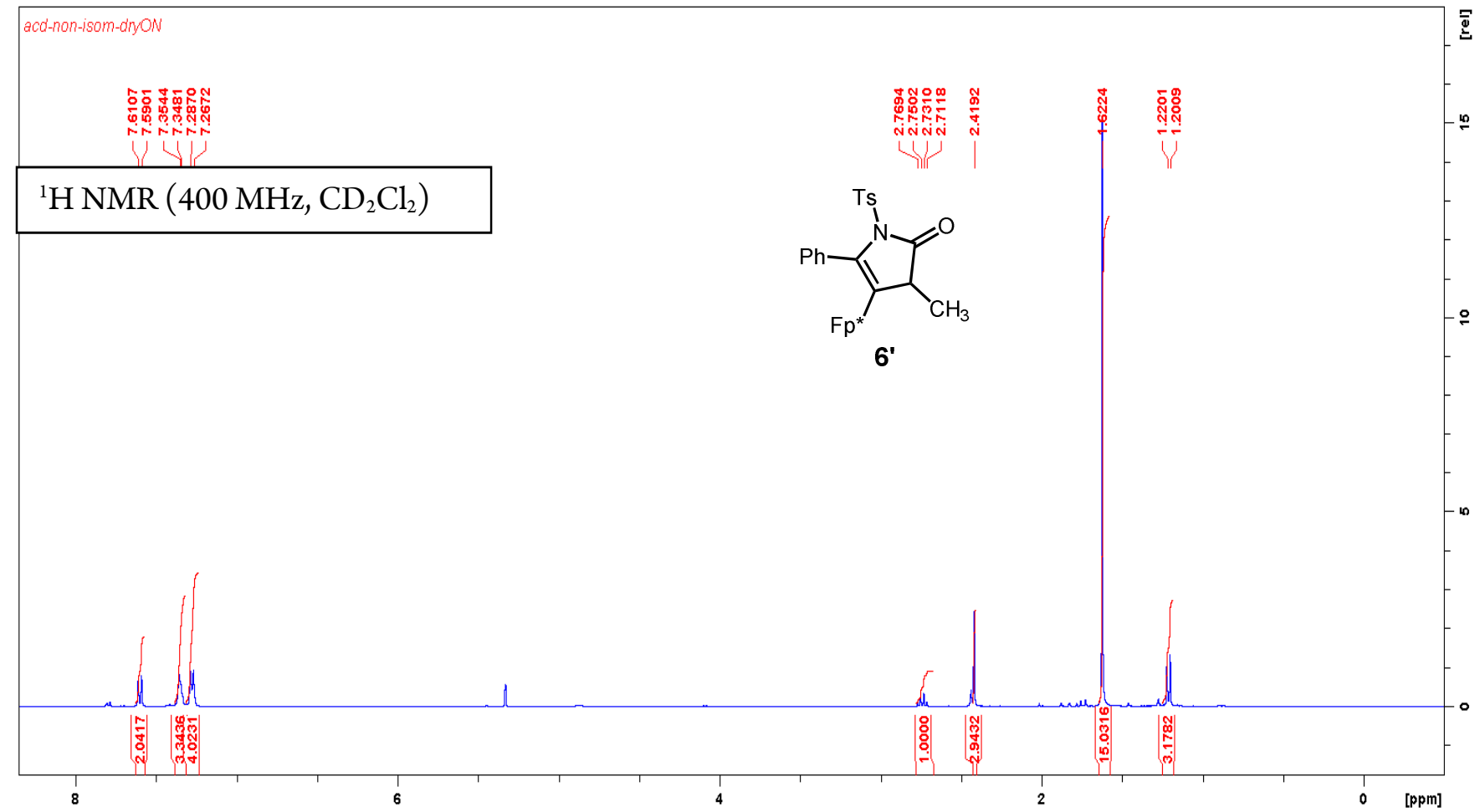

Figure S33: ${ }^{1} \mathrm{H}$ NMR spectrum of 6'

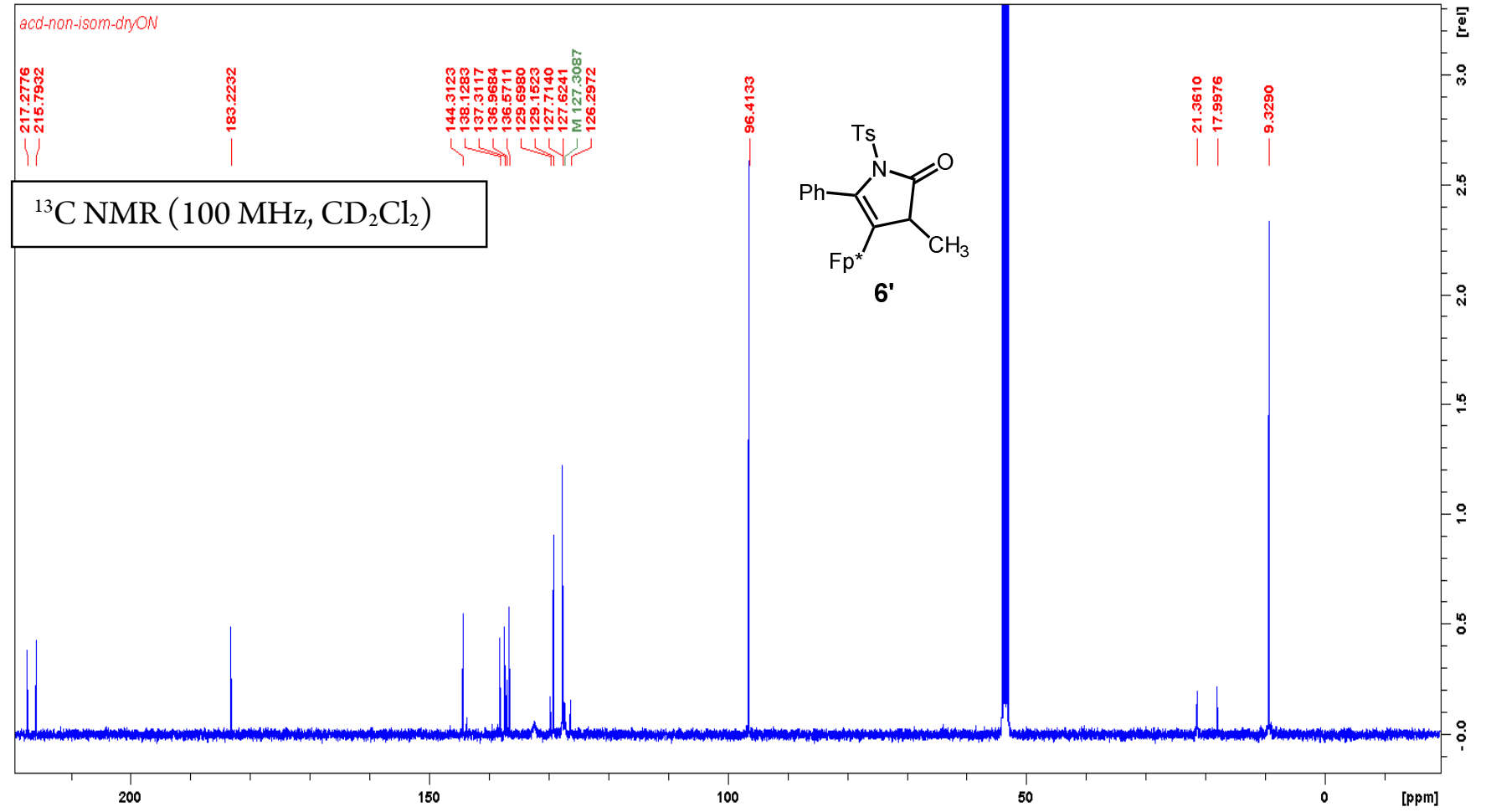

Figure S34: ${ }^{13} \mathrm{C}$ NMR spectrum of 6 ' 


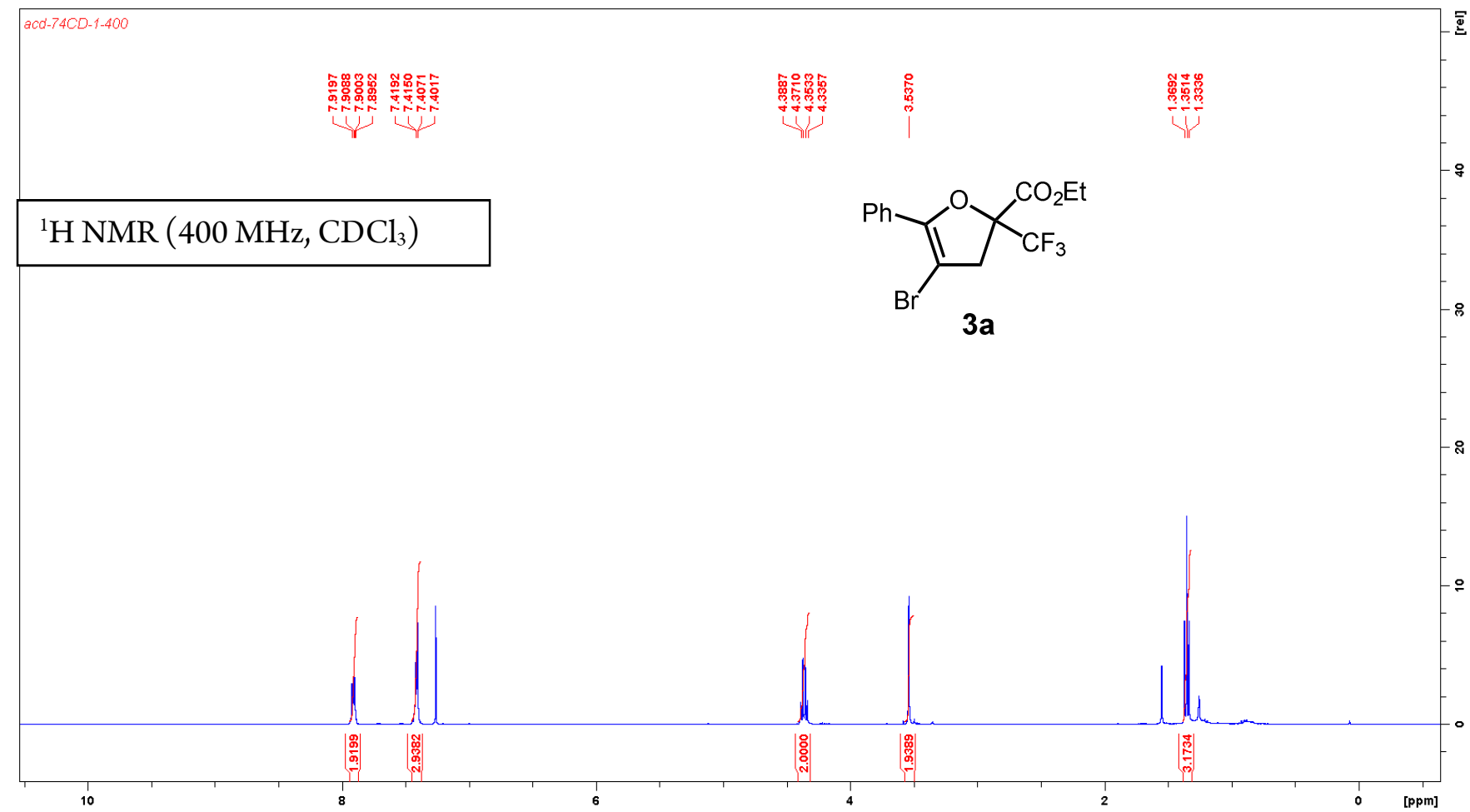

Figure S35: ${ }^{1} \mathrm{H}$ NMR spectrum of $\mathbf{3 a}$

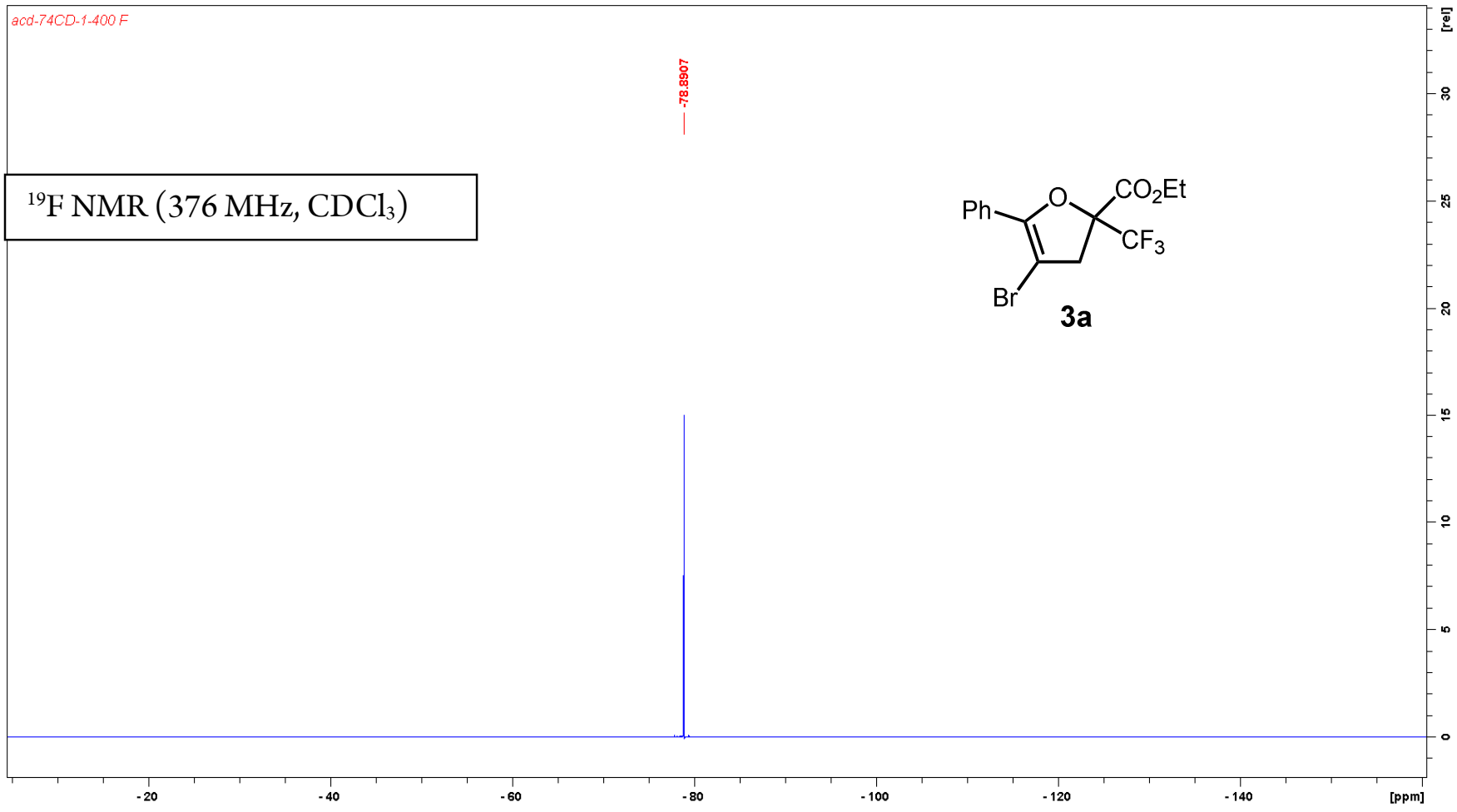

Figure S36: ${ }^{19} \mathrm{~F}$ NMR spectrum of $\mathbf{3 a}$ 


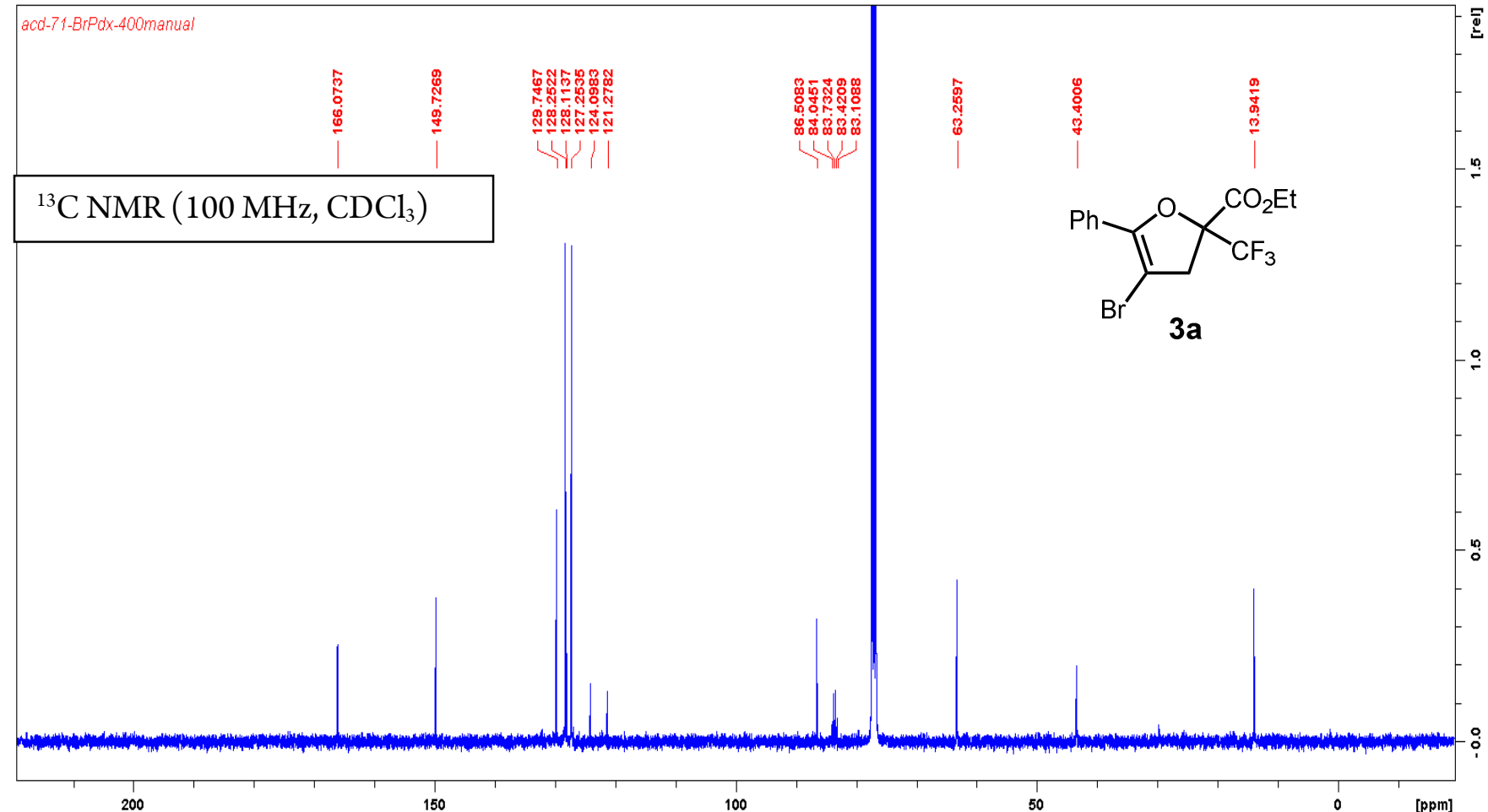

Figure S37: ${ }^{13} \mathrm{C}$ NMR spectrum of $3 \mathbf{a}$ 


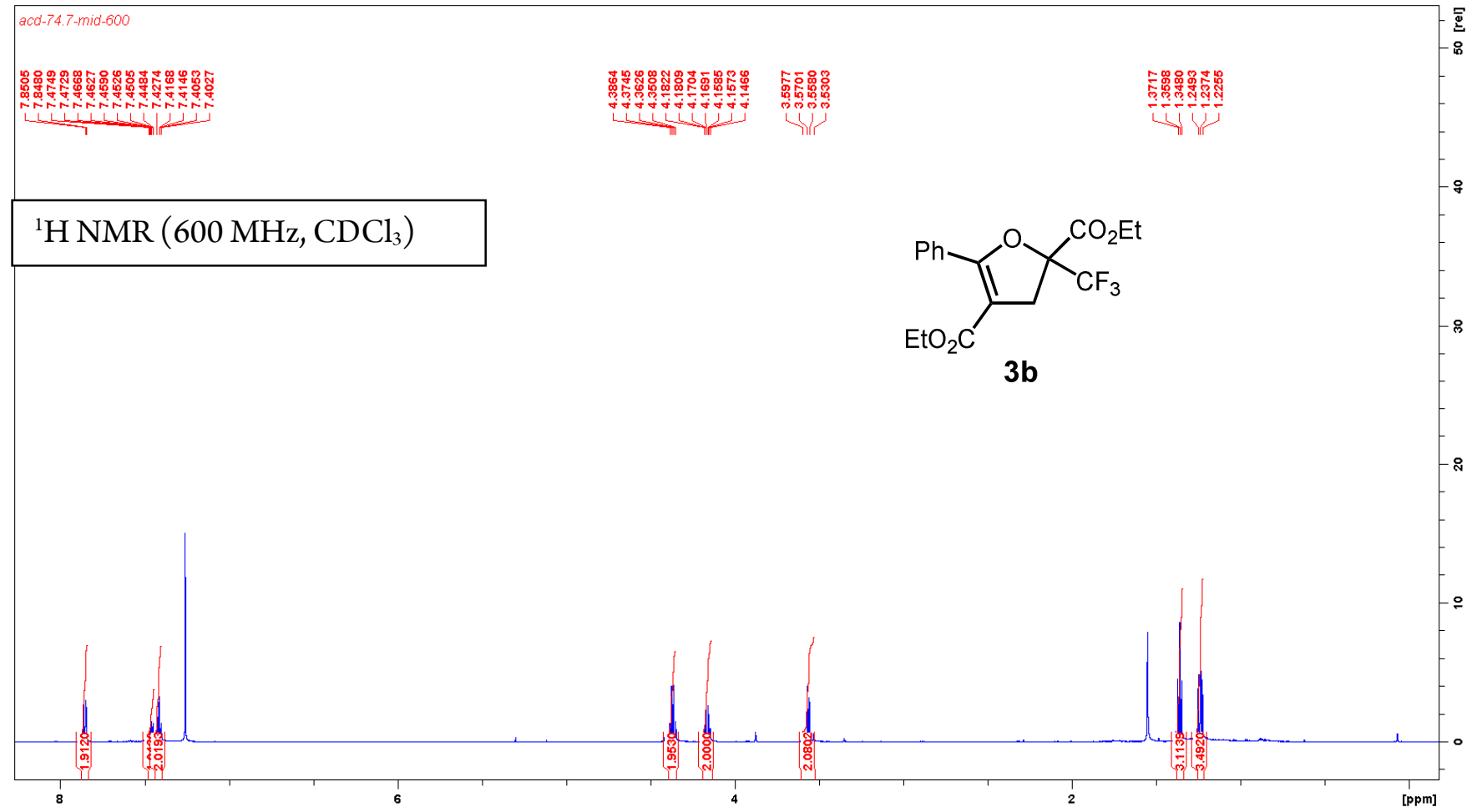

Figure S38: ${ }^{1} \mathrm{H}$ NMR spectrum of $\mathbf{3 b}$

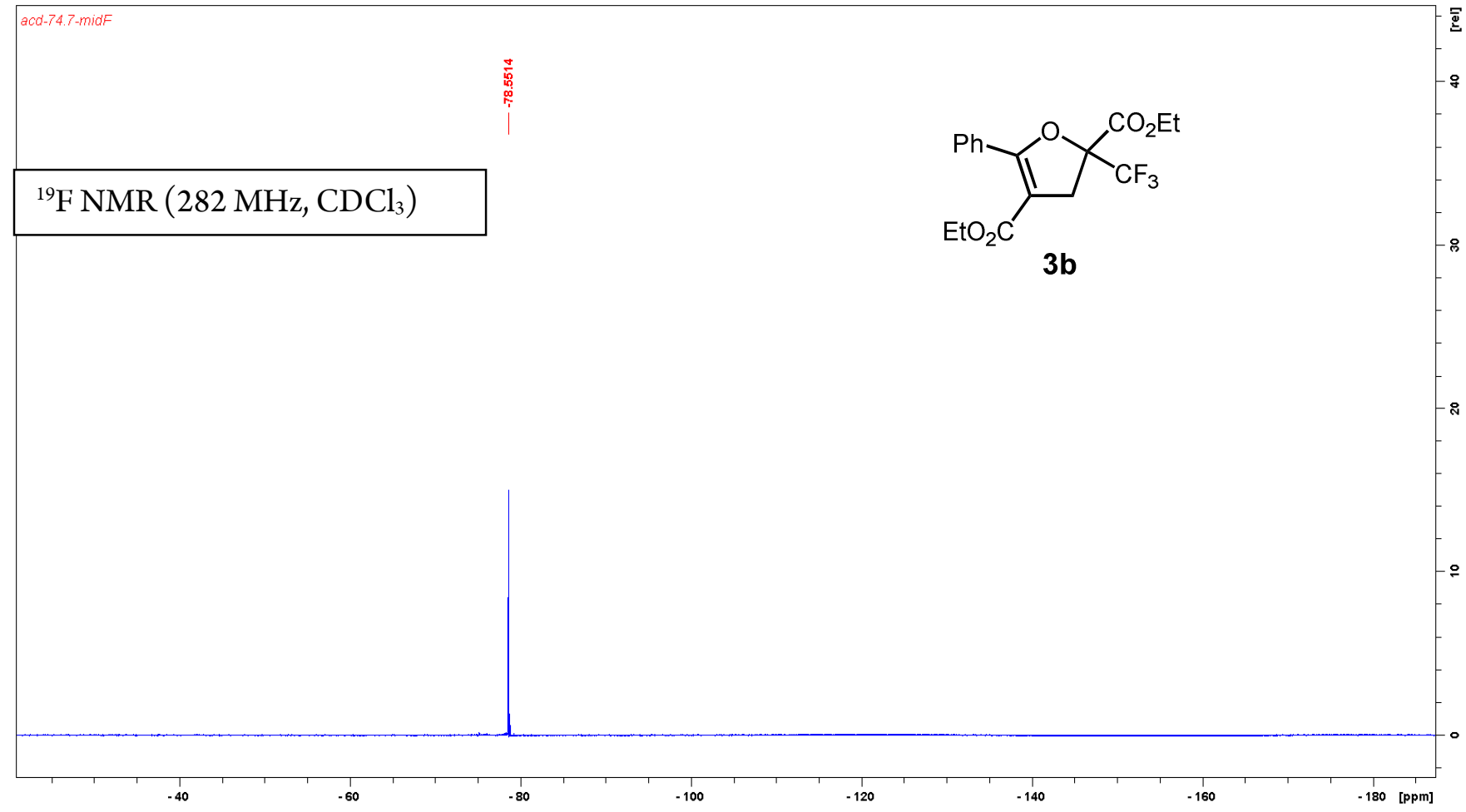

Figure S39: ${ }^{19} \mathrm{~F}$ NMR spectrum of $\mathbf{3 b}$ 


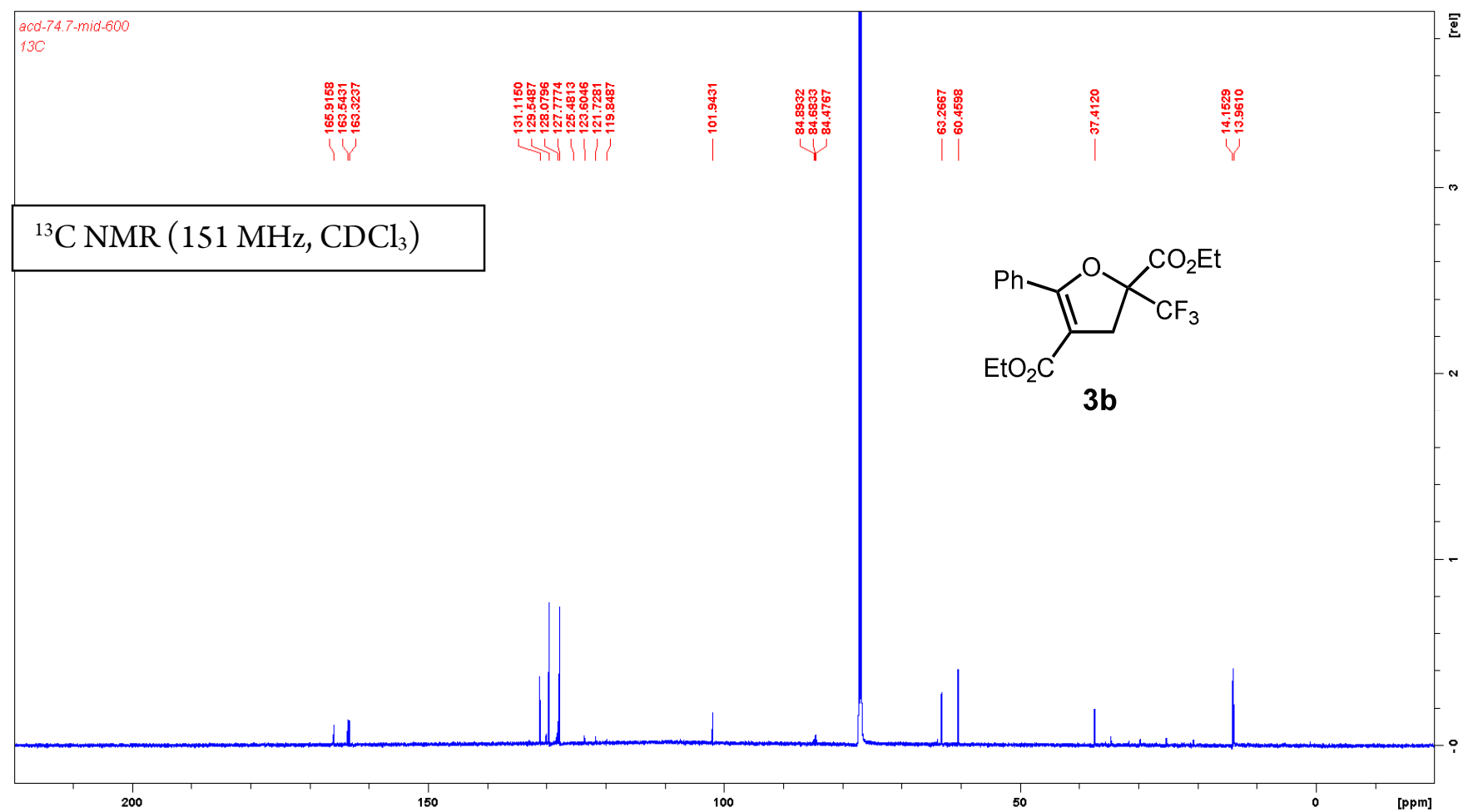

Figure S40: ${ }^{13} \mathrm{C}$ NMR spectrum of $\mathbf{3 b}$ 


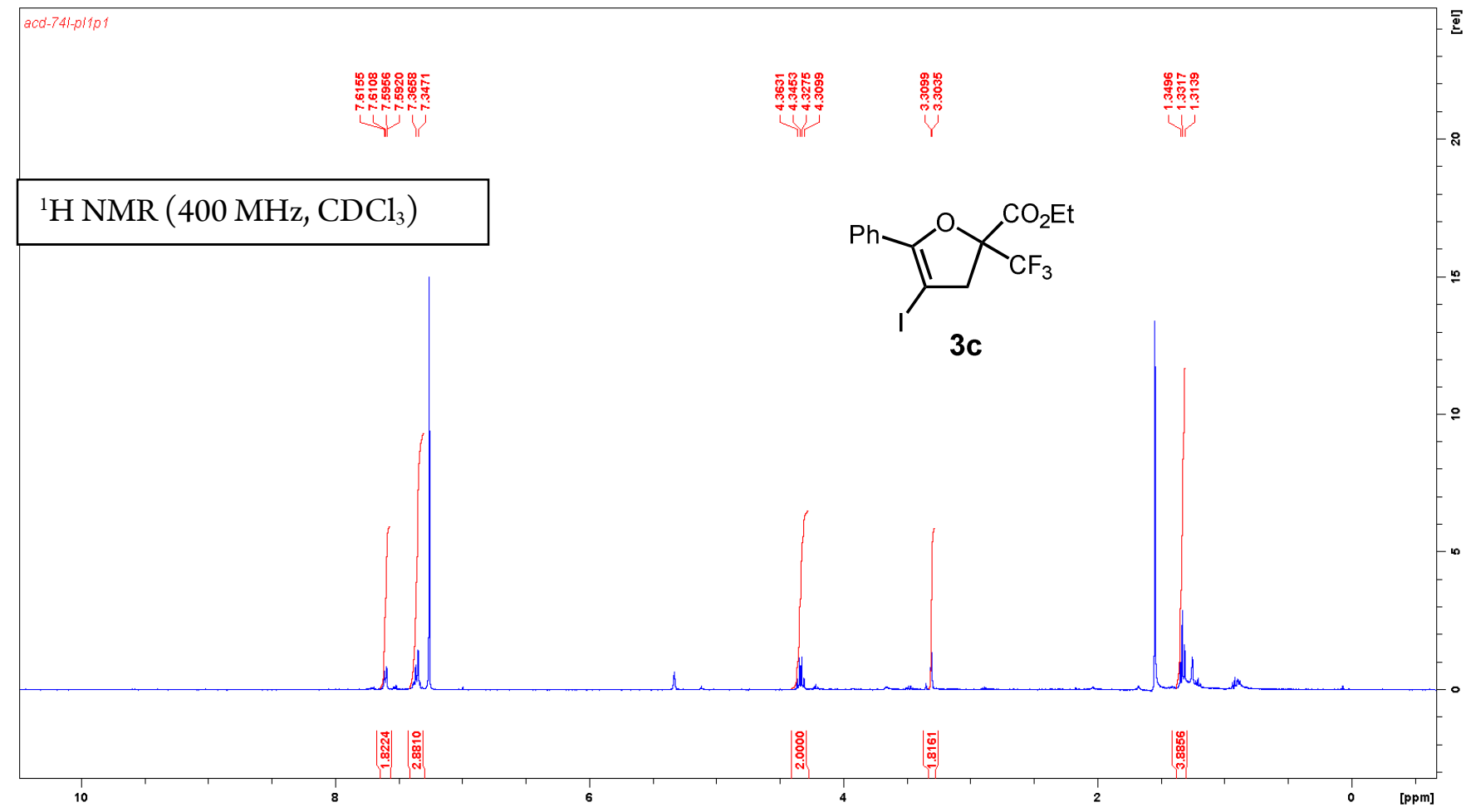

Figure S41: ${ }^{1} \mathrm{H}$ NMR spectrum of $3 \mathrm{c}$

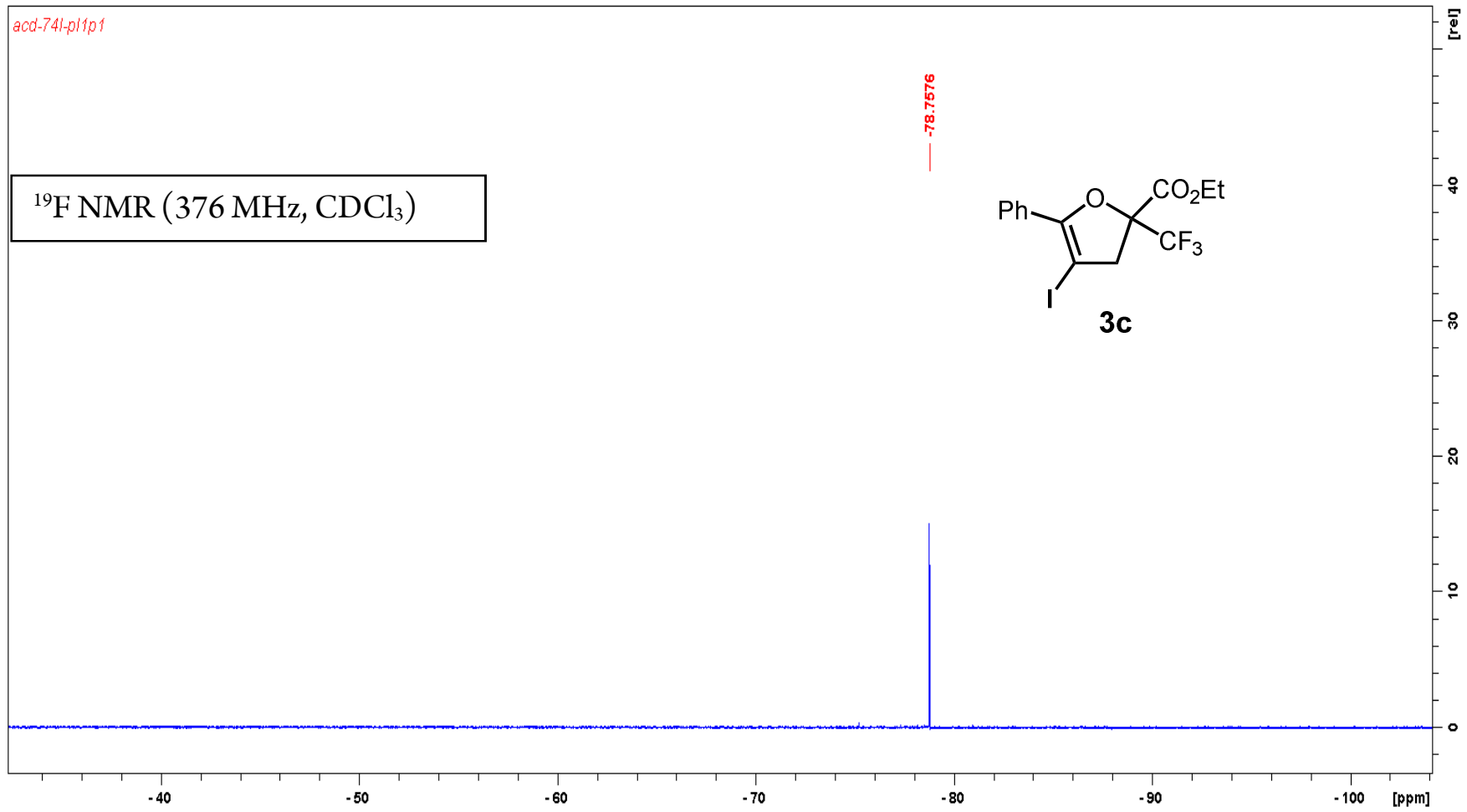

Figure S42: ${ }^{19} \mathrm{~F}$ NMR spectrum of 3c 


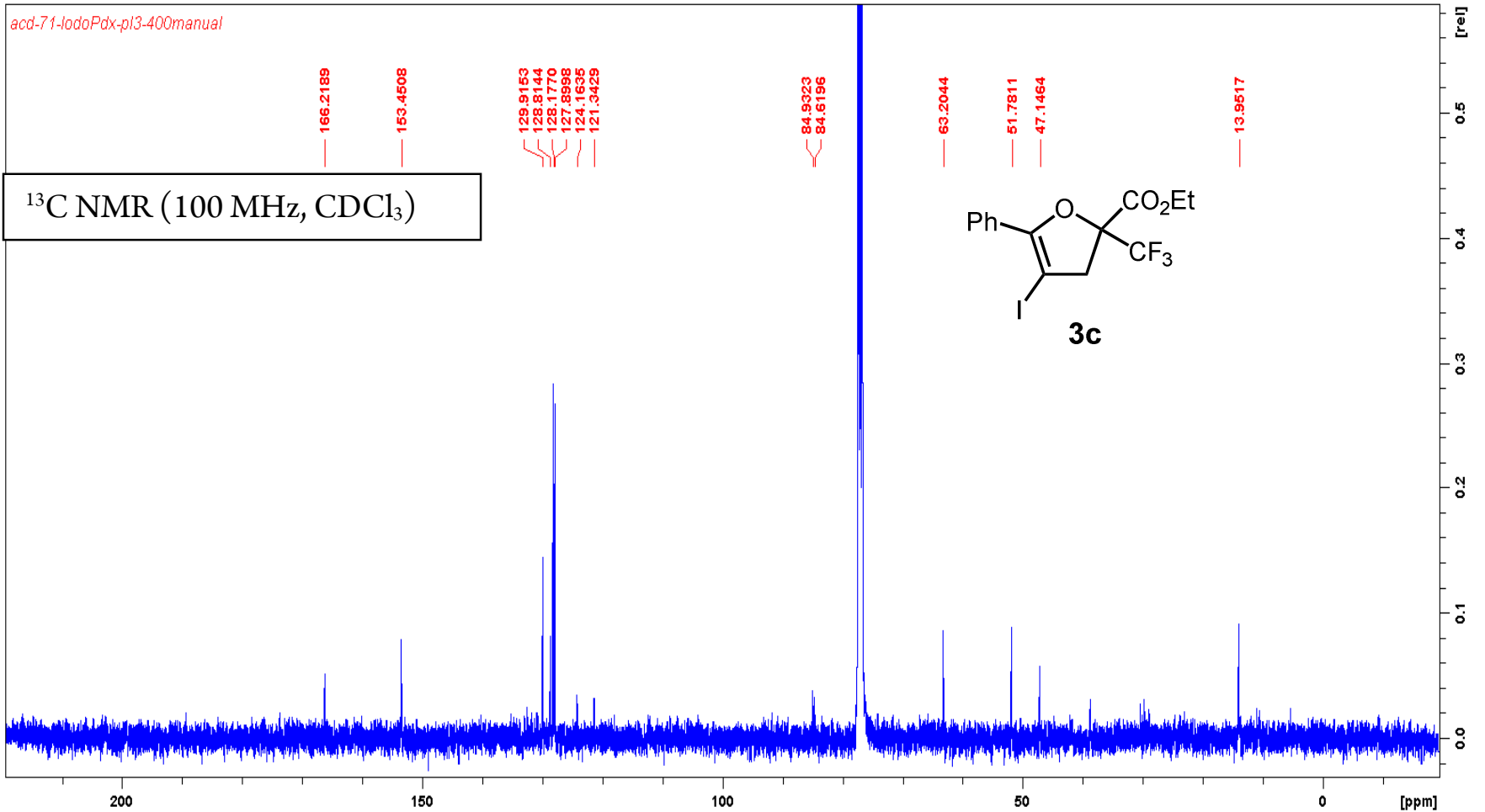

Figure S43: ${ }^{13} \mathrm{C}$ NMR spectrum of $3 \mathrm{c}$ 


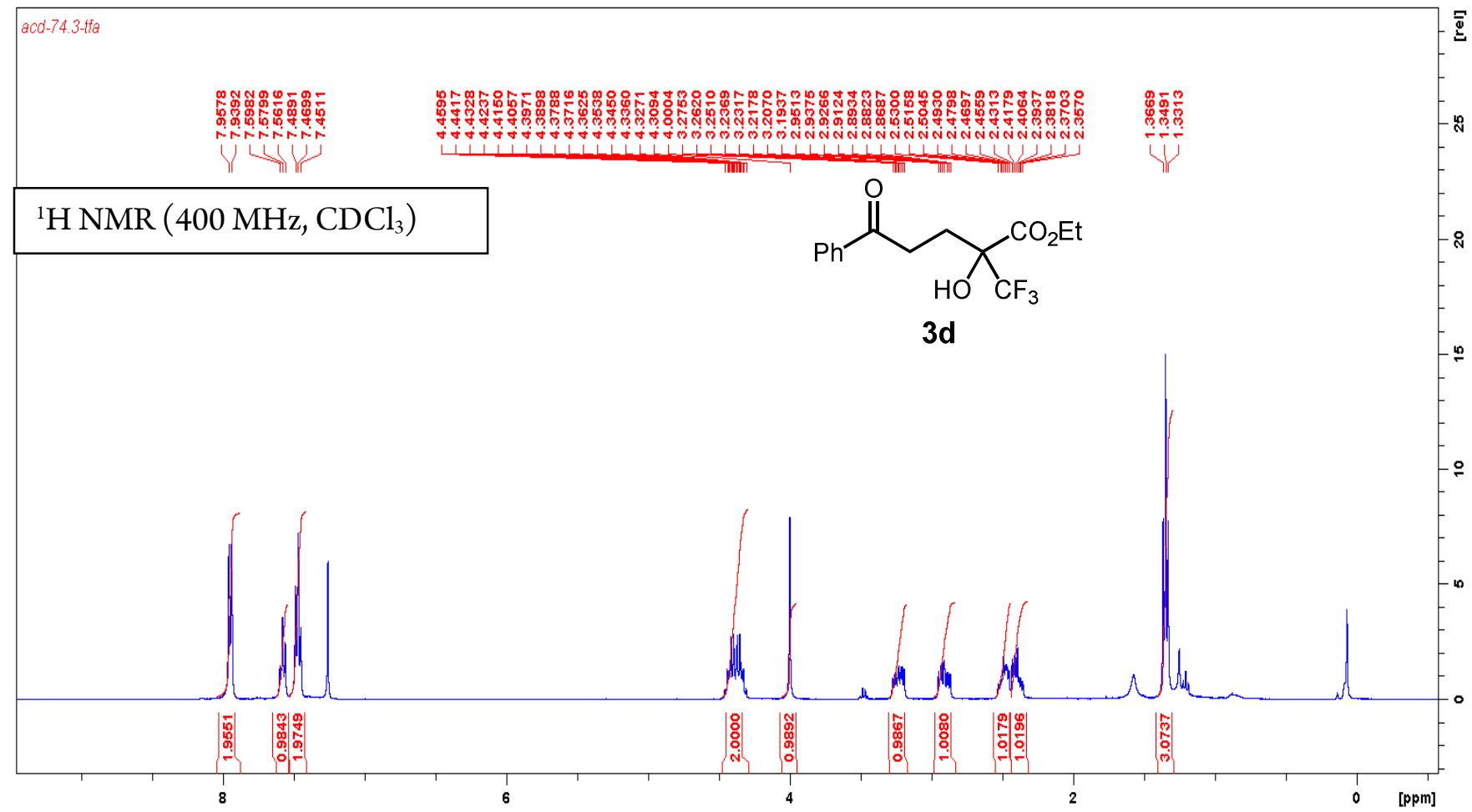

Figure S44: ${ }^{1} \mathrm{H}$ NMR spectrum of $\mathbf{3 d}$

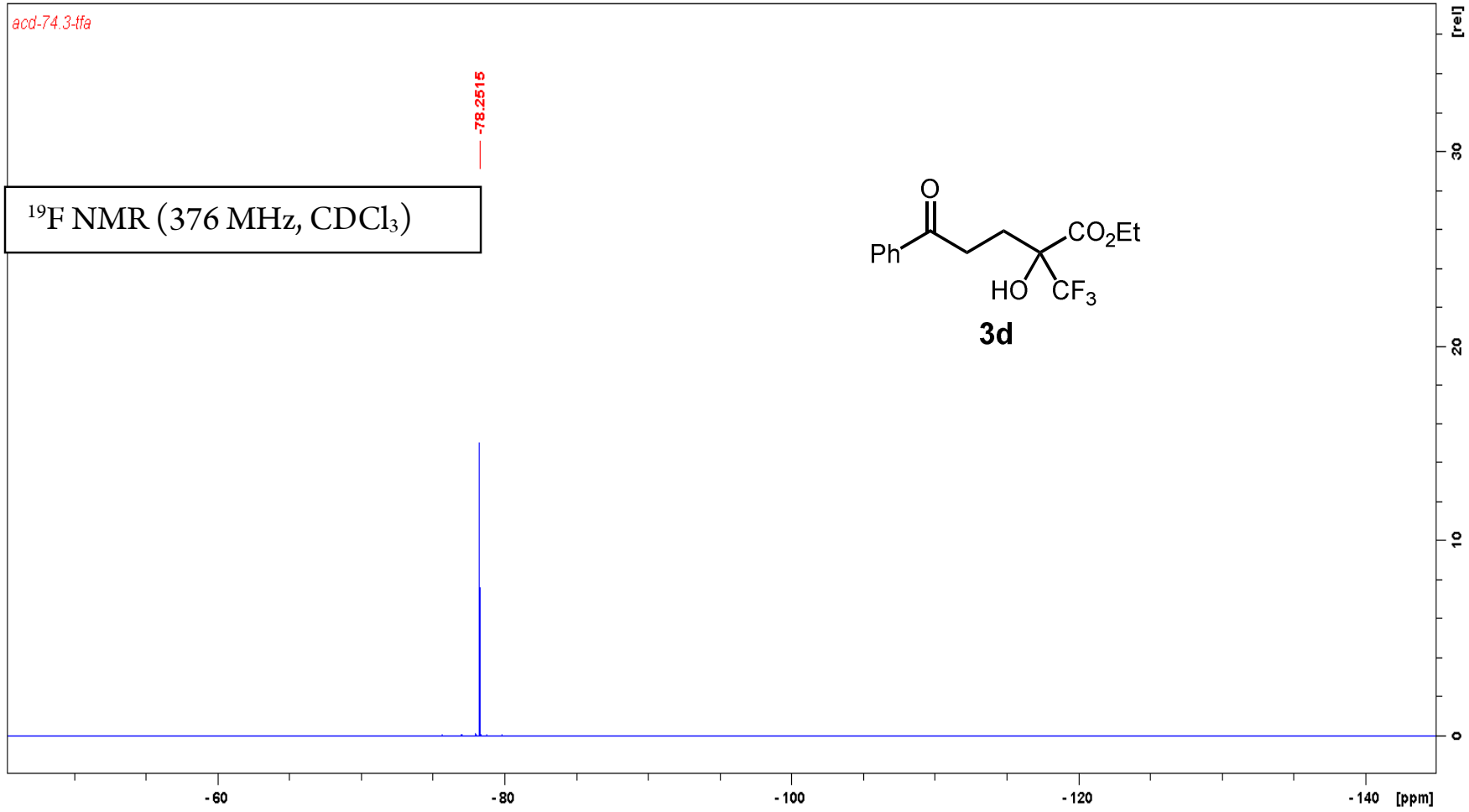

Figure S45: ${ }^{19} \mathrm{~F}$ NMR spectrum of $\mathbf{3 d}$ 


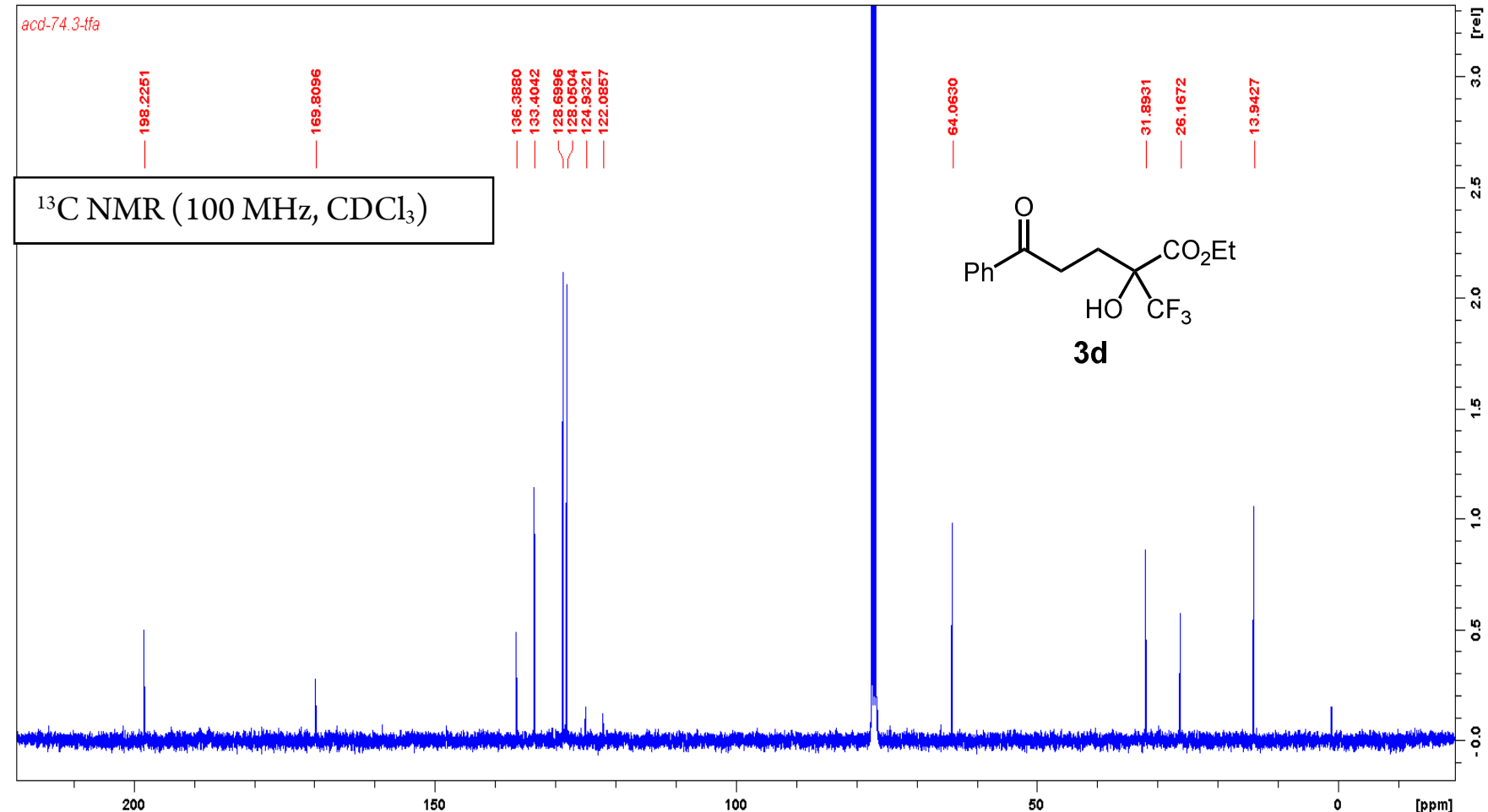

Figure S46: ${ }^{13} \mathrm{C}$ NMR spectrum of 3d 


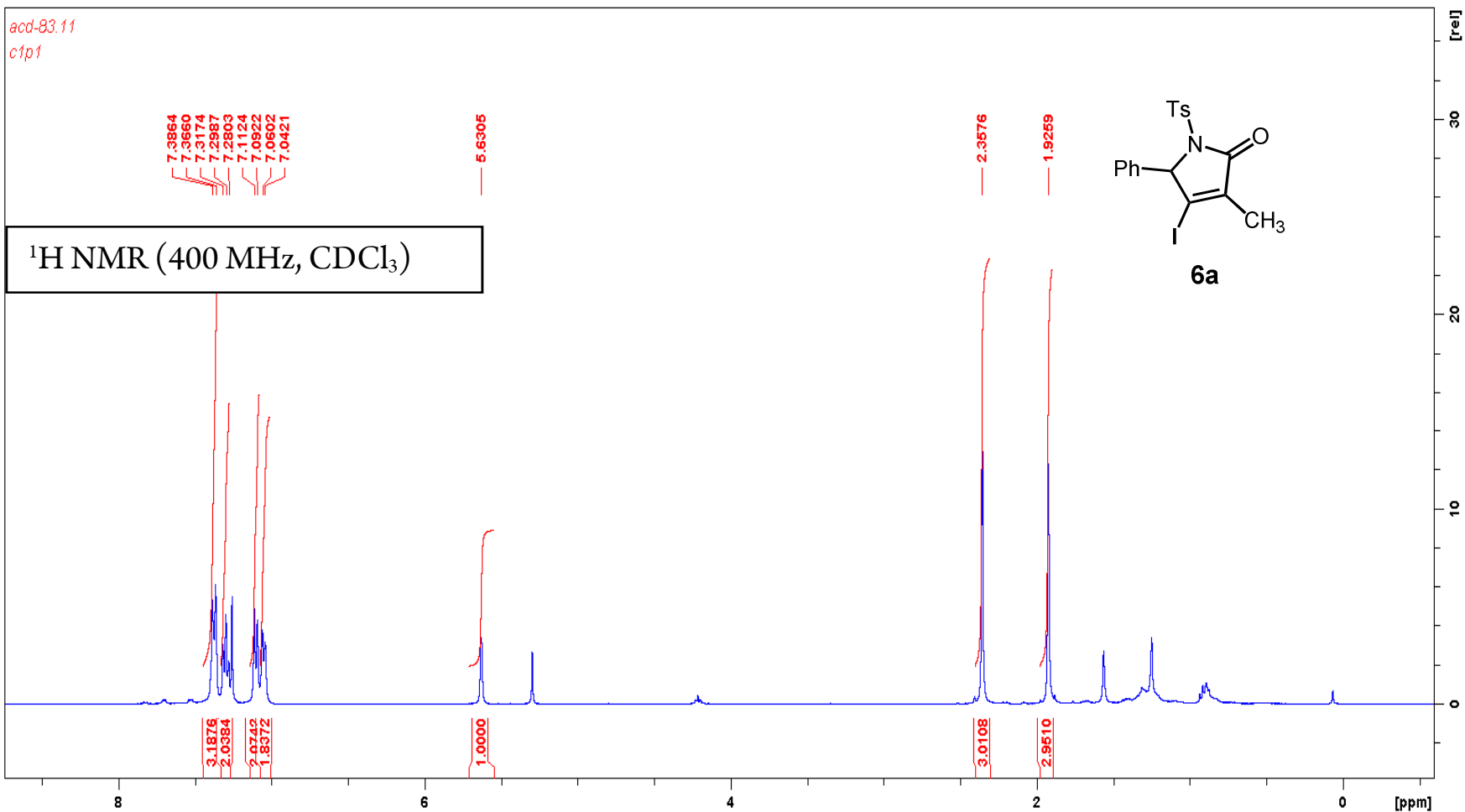

Figure S47: ${ }^{1} \mathrm{H}$ NMR spectrum of $\mathbf{6 a}$

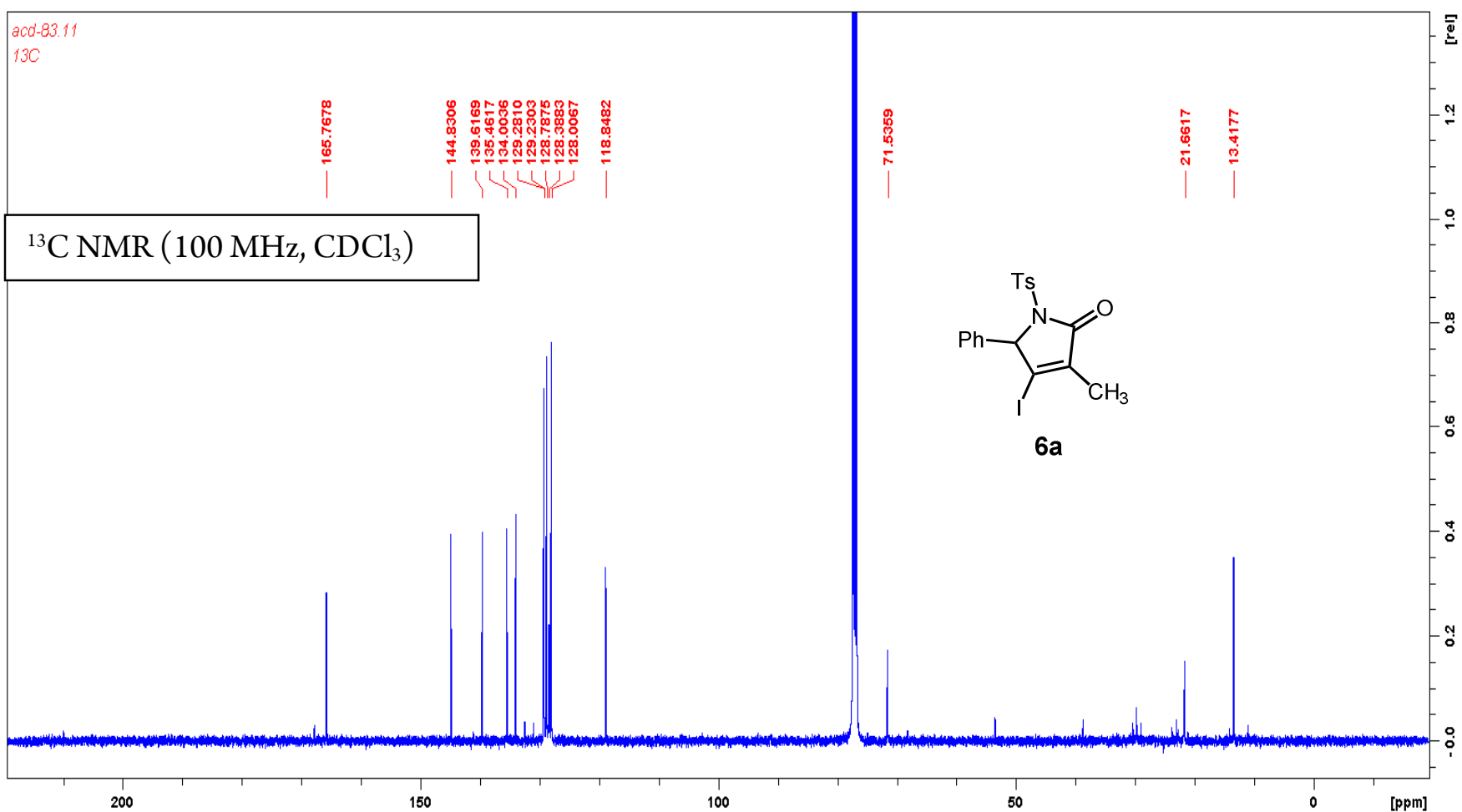

Figure S48: ${ }^{13} \mathrm{C}$ NMR spectrum of $6 a$ 


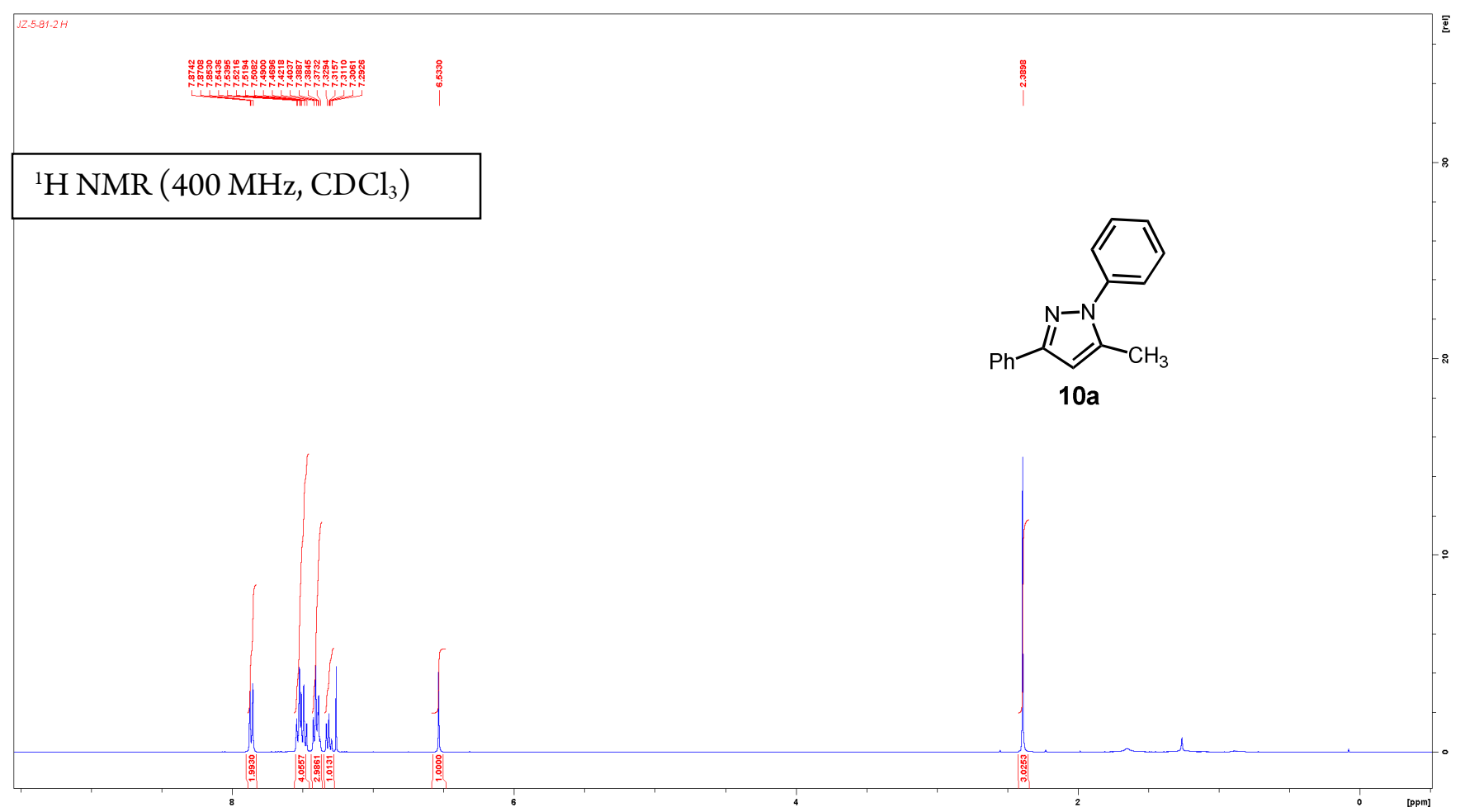

Figure S49: ${ }^{1} \mathrm{H}$ NMR spectrum of $\mathbf{1 0 a}$

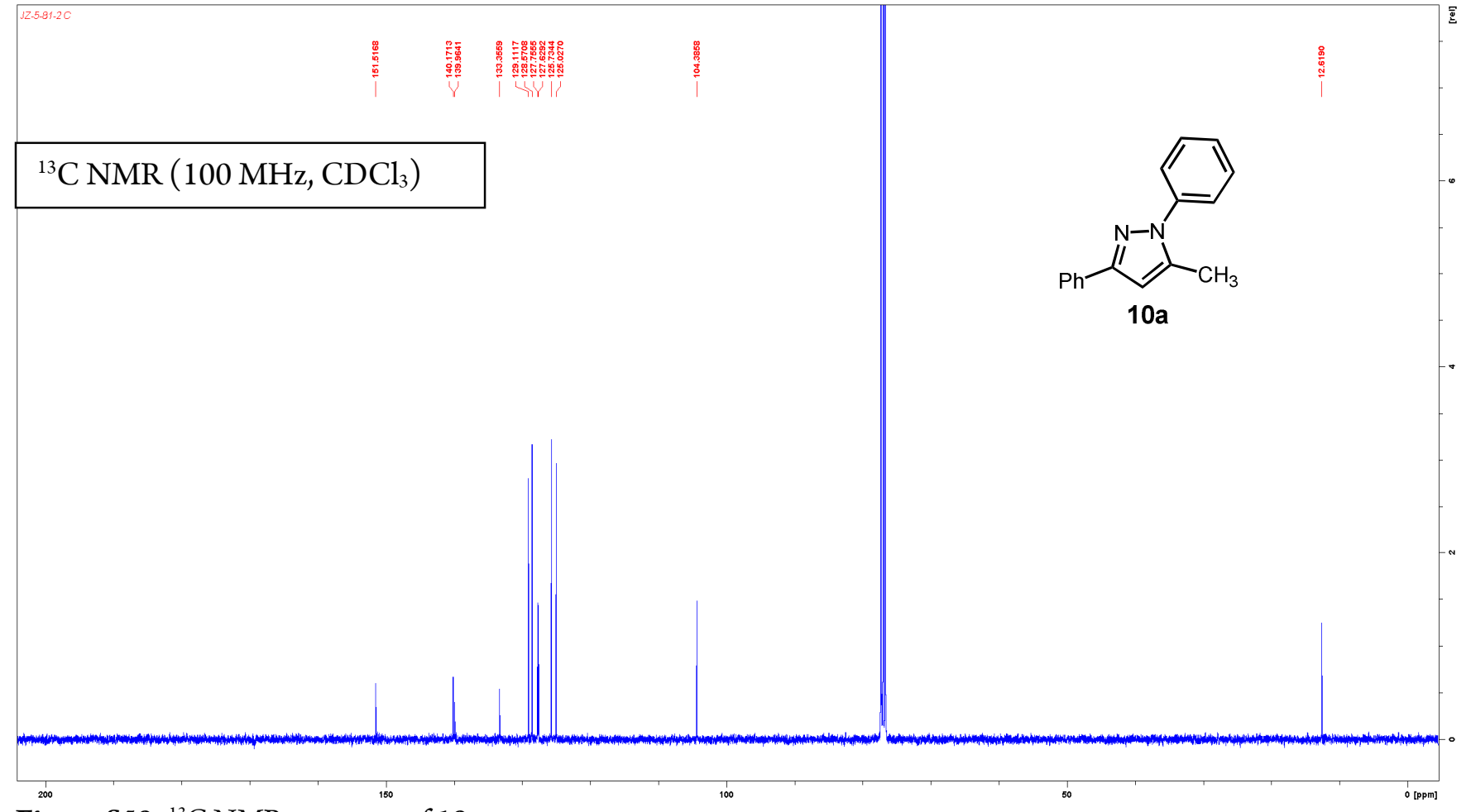

Figure S50: ${ }^{13} \mathrm{C}$ NMR spectrum of $10 a$ 


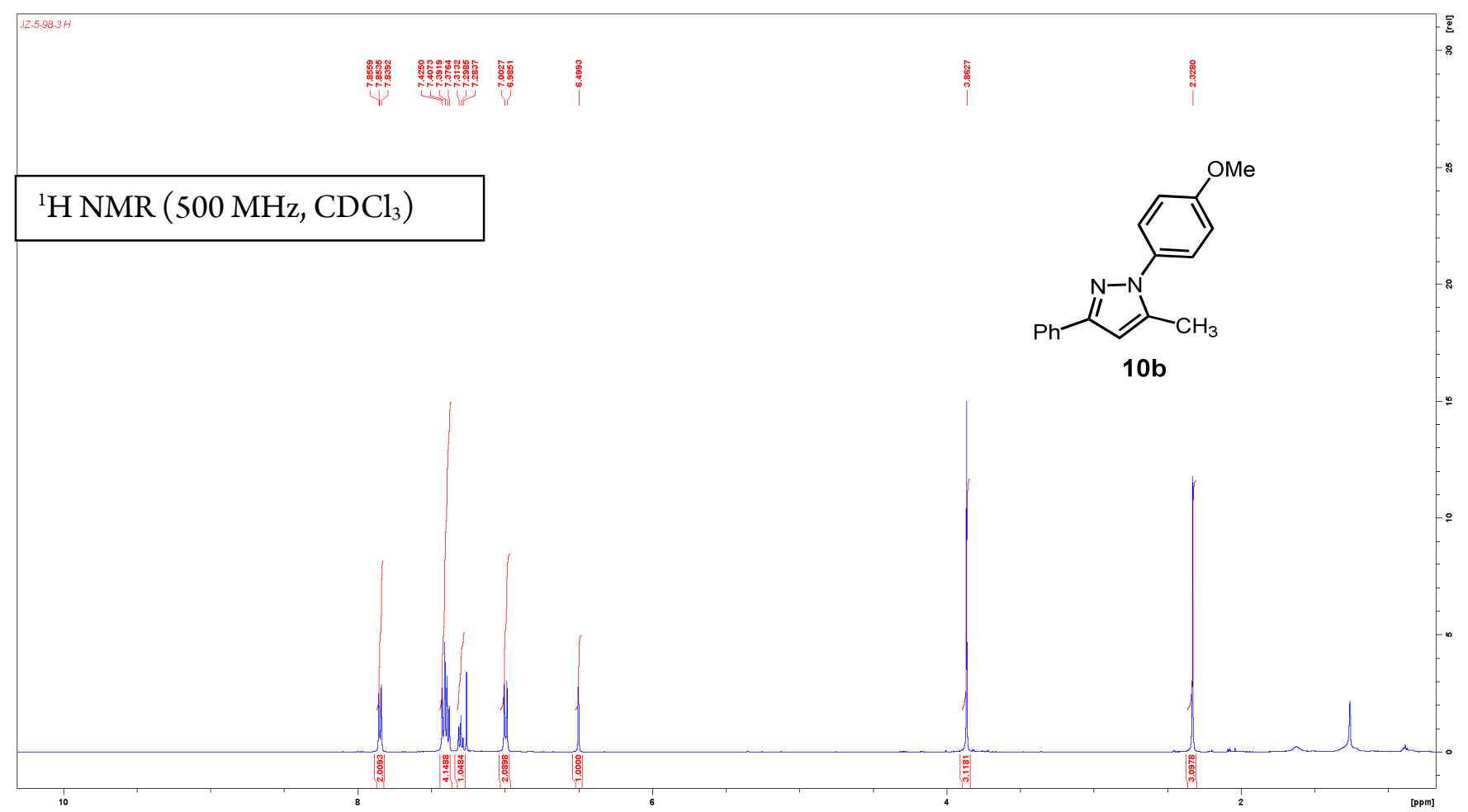

Figure S51: ${ }^{1} \mathrm{H}$ NMR spectrum of $\mathbf{1 0 b}$

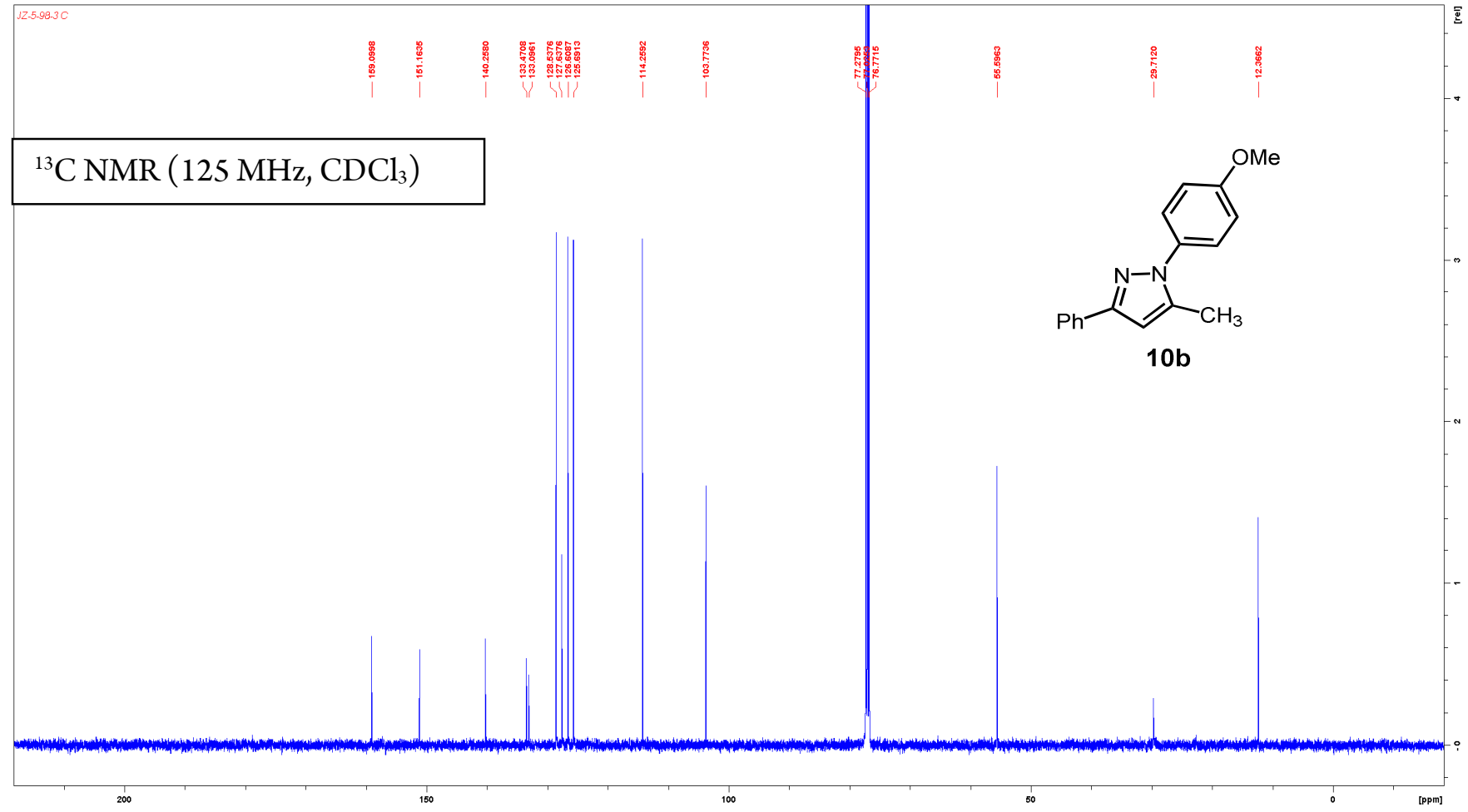

Figure S52: ${ }^{13} \mathrm{C}$ NMR spectrum of $\mathbf{1 0 b}$ 


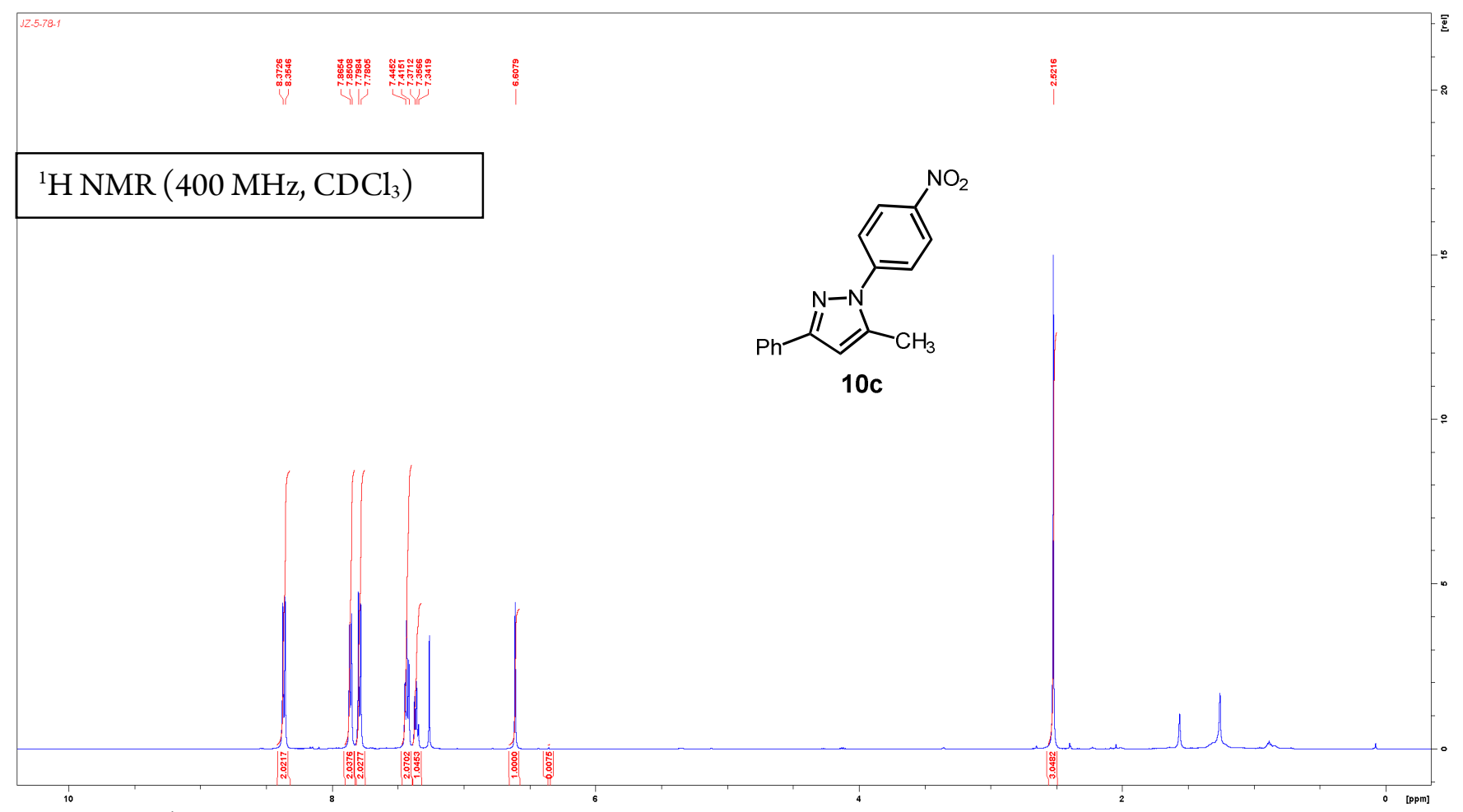

Figure S53: ${ }^{1} \mathrm{H}$ NMR spectrum of 10c

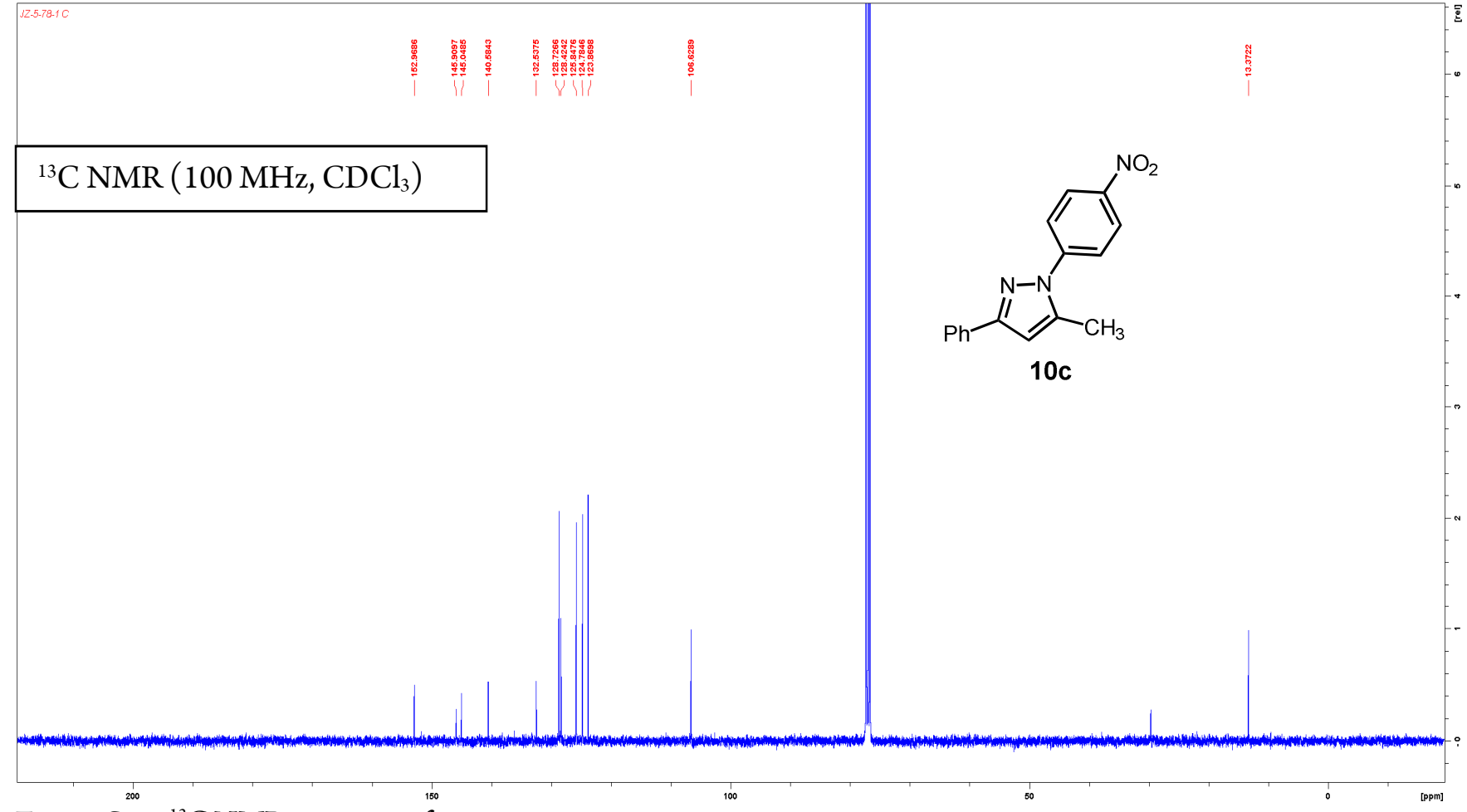

Figure S54: ${ }^{13} \mathrm{C}$ NMR spectrum of 10c 


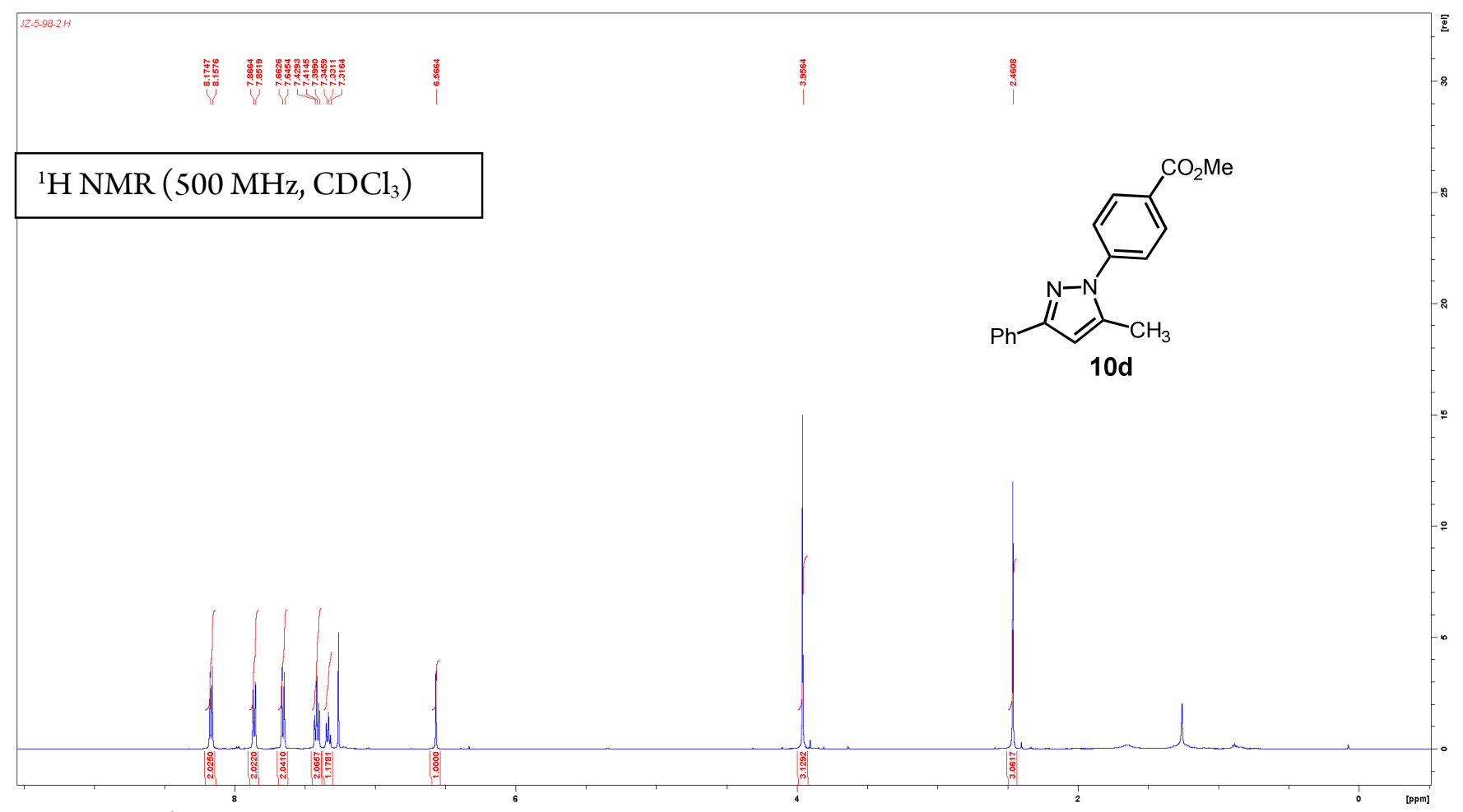

Figure S55: ${ }^{1} \mathrm{H}$ NMR spectrum of $10 d$

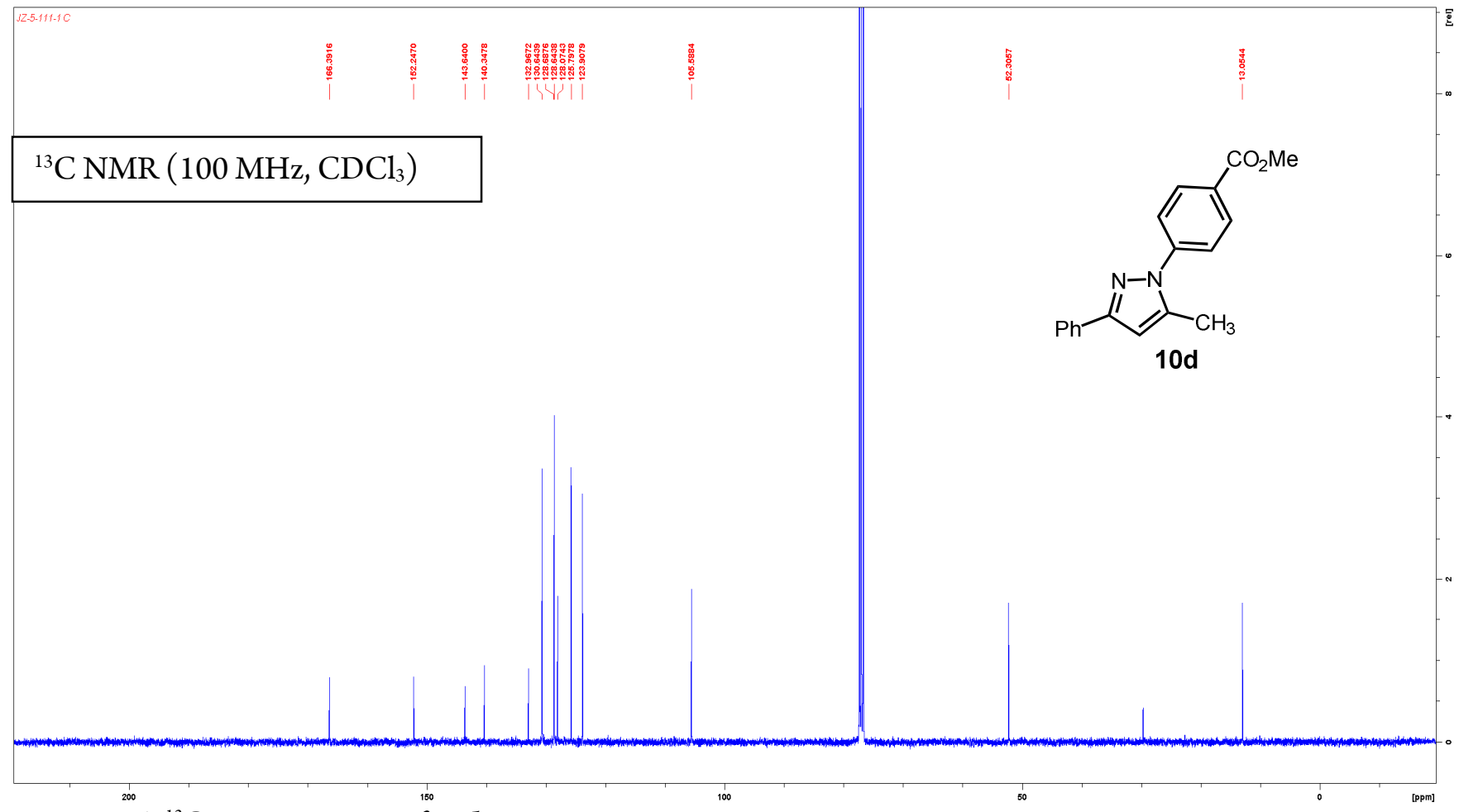

Figure S56: ${ }^{13} \mathrm{C}$ NMR spectrum of $10 \mathrm{~d}$ 


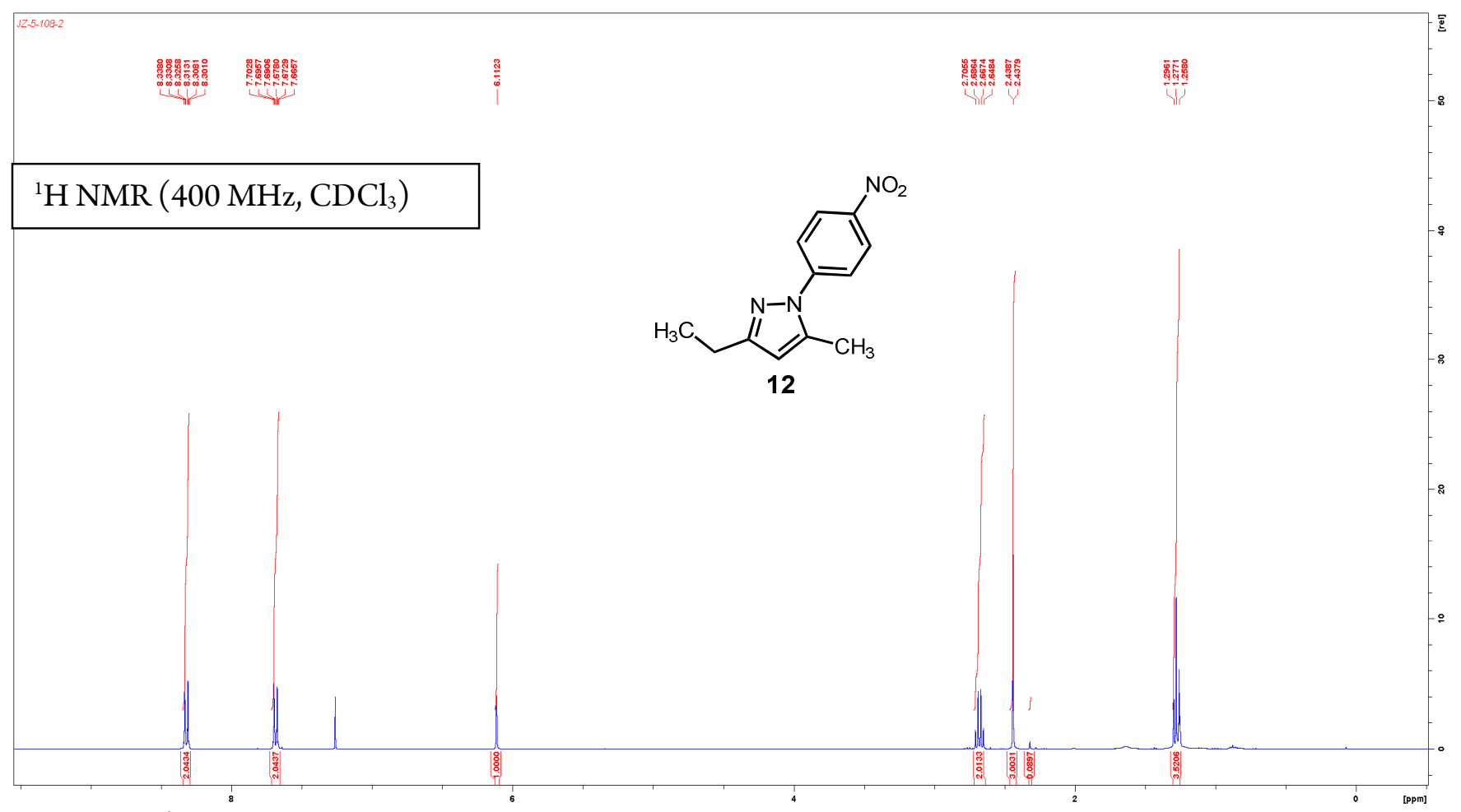

Figure S57: ${ }^{1} \mathrm{H}$ NMR spectrum of 12

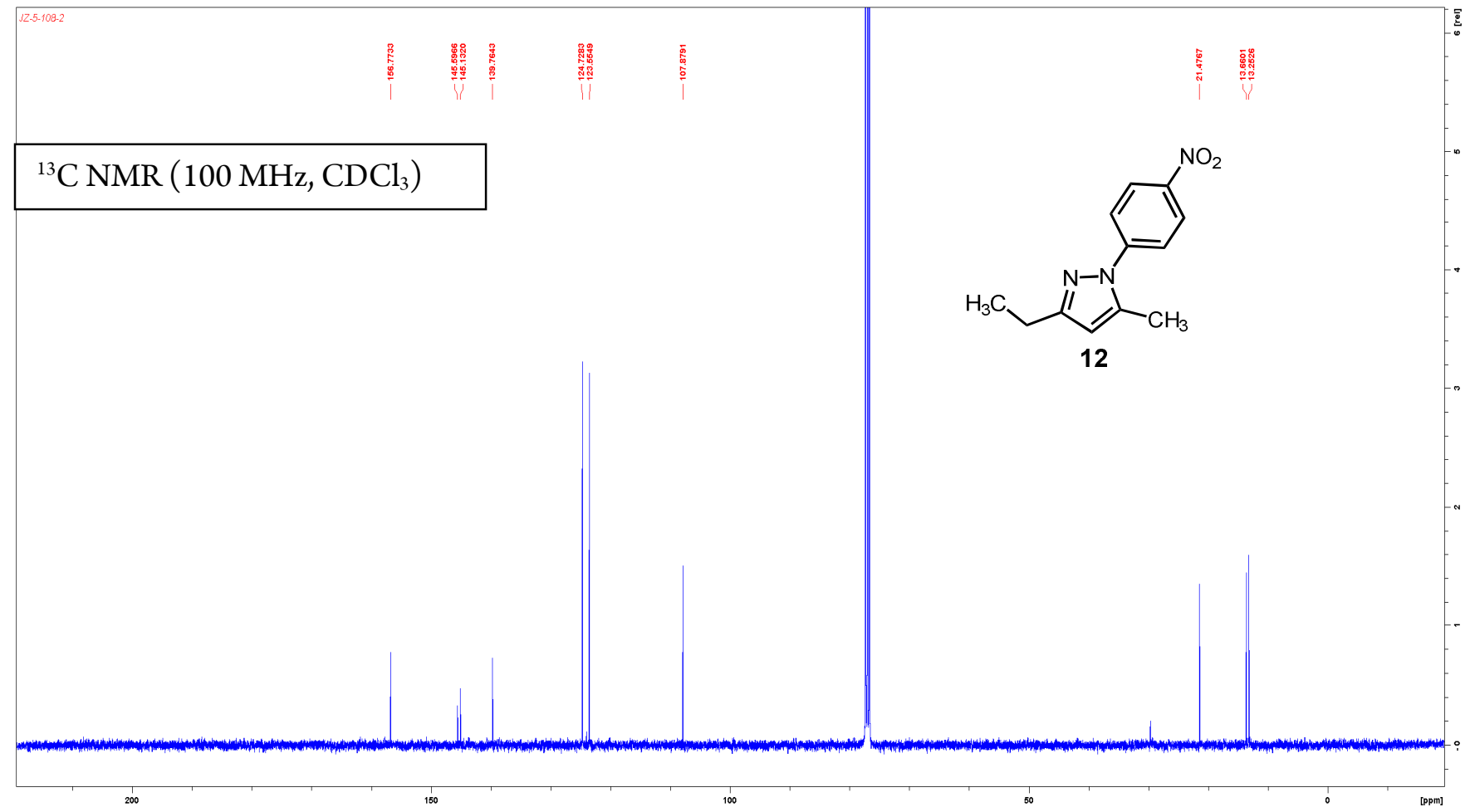

Figure S58: ${ }^{13} \mathrm{C}$ NMR spectrum of 12 


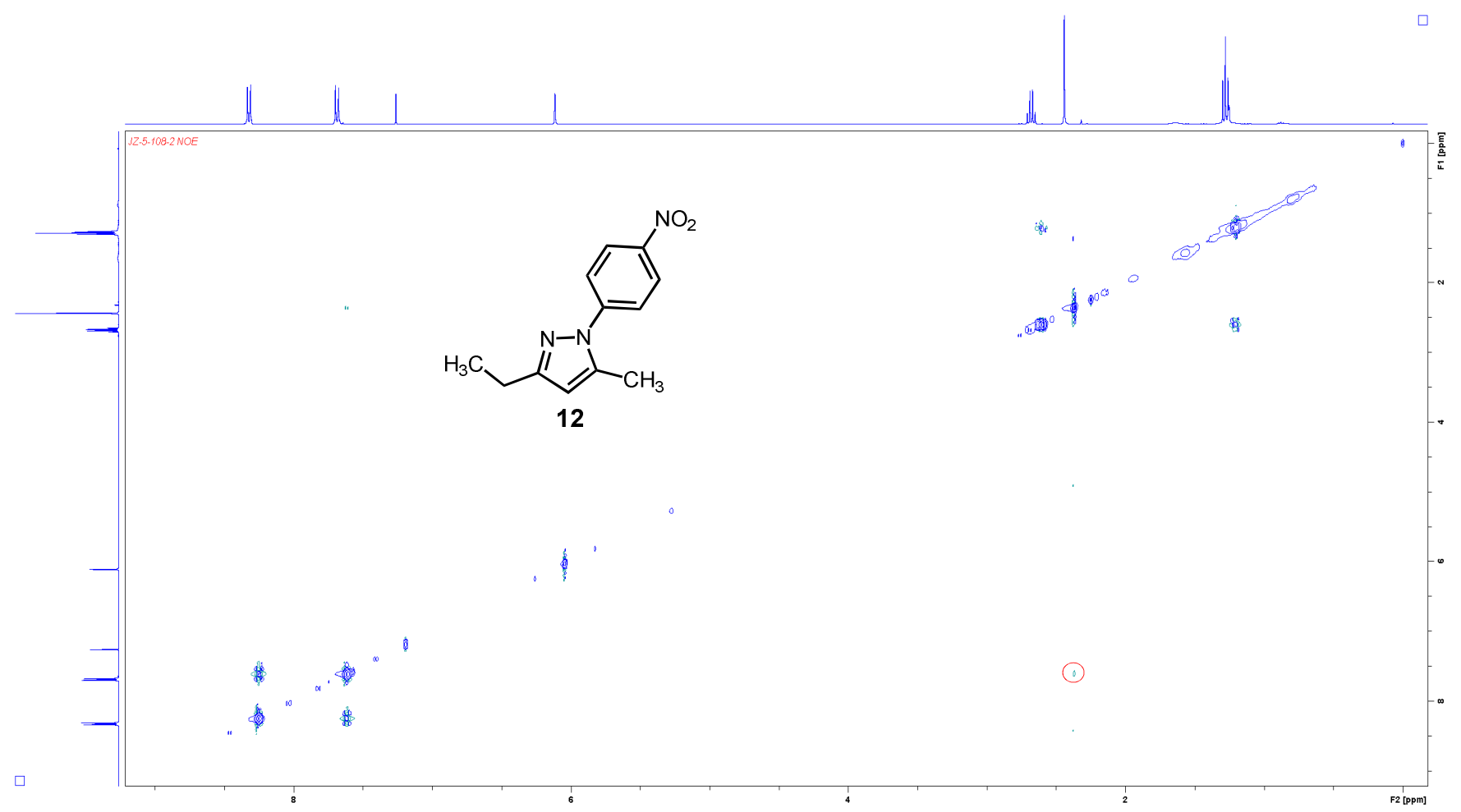

Figure S59: 2D NOESY spectrum of 12 


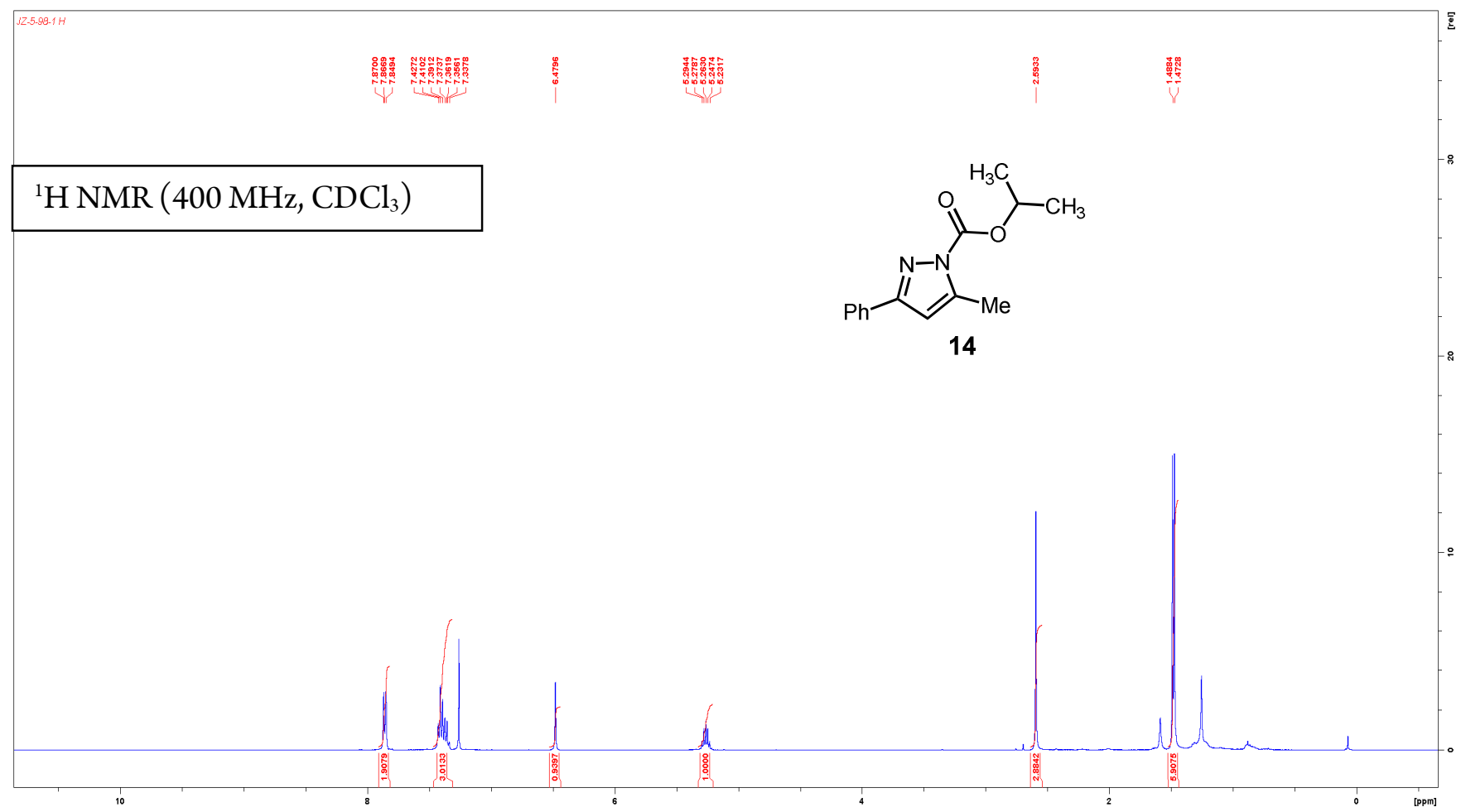

Figure S60: ${ }^{1} \mathrm{H}$ NMR spectrum of 14

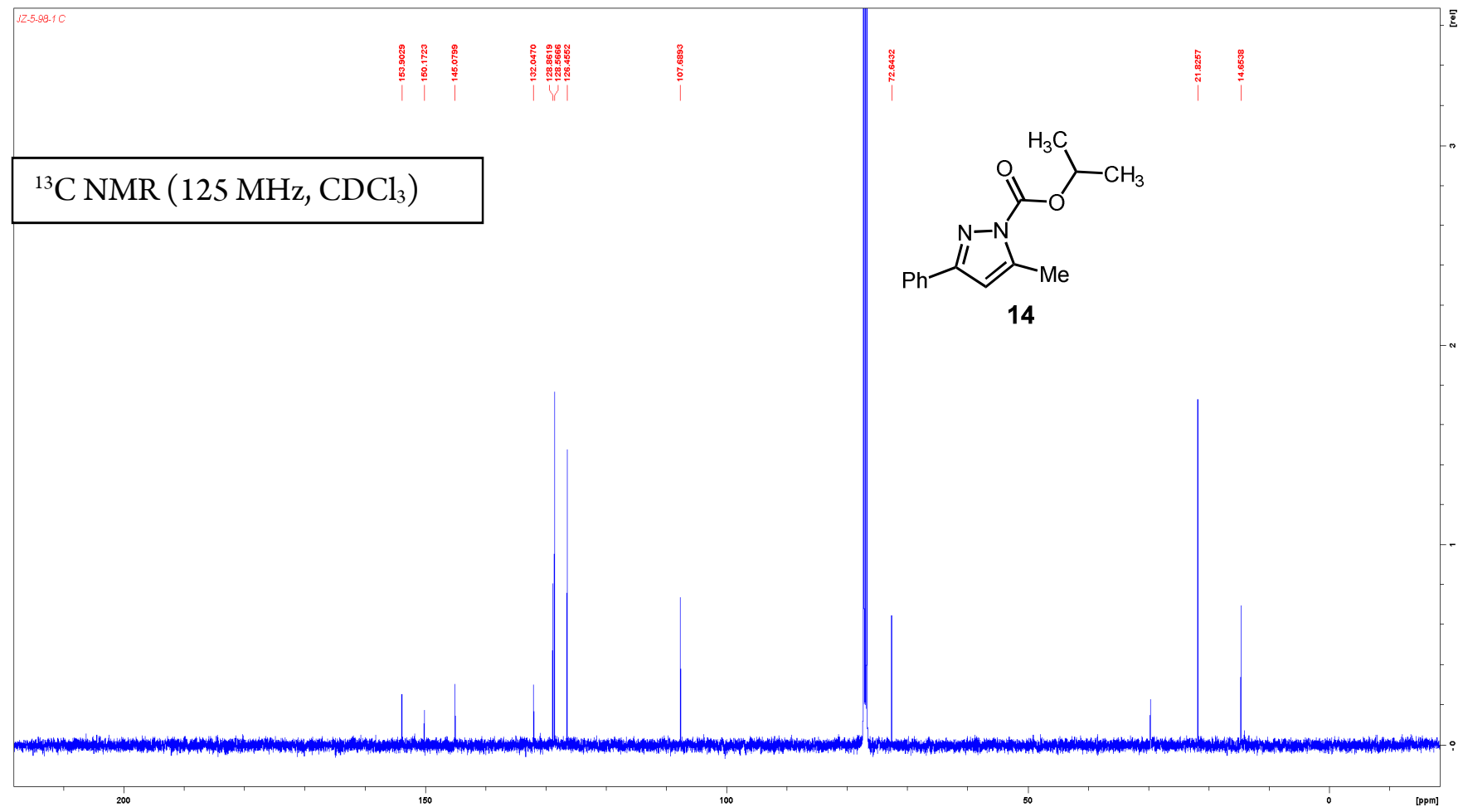

Figure S61: ${ }^{13} \mathrm{C}$ NMR spectrum of 14 


\section{References}

1. Wang, Y.; Zhu, J.; Durham, A. C.; Lindberg, H.; Wang, Y.-M., $\alpha-C-H$ Functionalization of $\pi$-Bonds Using Iron Complexes: Catalytic Hydroxyalkylation of Alkynes and Alkenes. J. Am. Chem. Soc. 2019, 141 (50), 19594-19599.

2.Sung Chun, Y.; Kon Lee, K.; Ok Ko, Y.; Shin, H.; Lee, S.-g., The first chemoselective tandem acylation of the Blaise reaction intermediate: a novel method for the synthesis of $\alpha$-acyl- $\beta$-enamino esters, key intermediate for pyrazoles. Chem. Commun. 2008, (41), 5098-5100.

3. Alberola, A.; Bleye, L. C., González-Ortega, A.; Sádaba, M. L; Sañud, M. C., Scope and Limitations in the Regioselective Synthesis of 1,3,5-Trisubstituted Pyrazoles from $\beta$-Amino Enones and Hydrazine Derivatives. ${ }^{13} \mathrm{C}$-Chemical Shift Prediction Rules for 1,3,5-Trisubstituted Pyrazoles. Heterocycles 1970, 55 (2), 331-351.

4. Khairnar, P. V.; Lung, T.-H.; Lin, Y.-J.; Wu, C.-Y.; Koppolu, S. R.; Edukondalu, A.; Karanam, P.; Lin, W., An Intramolecular Wittig Approach toward Heteroarenes: Synthesis of Pyrazoles, Isoxazoles, and Chromenone-oximes. Org. Lett. 2019, 21 (11), 4219-4223.

5. (a) Wen, J.; Fu, Y.; Zhang, R.-Y.; Zhang, J.; Chen, S.-Y.; Yu, X.-Q., A simple and efficient synthesis of pyrazoles in water. Tetrahedron 2011, 67 (49), 9618-9621. (b) Kashima, C.; Harada, H.; Kita, I.; Fukuchi, I,; Hosomi, A., The preparation of N-acylpyrazoles and their behavior toward alcohol. Synthesis 1994, 1994 (1), 61-65. (c) Faure, R.; Vincent, É.-J.; Rousseau, A.; Claramunt, R. M.; Elguero, J., High resolution 13C nuclear magnetic resonance spectra of solid pyrazoles. Application to annular tautomerism. Can. J. Chem. 1988, 66 (5), 1141-1146. (d) Katritzky, A.R.; Ramsden, C.A.; Joule, J.A.; Zhdankin, V.V. Reactivity of Five-membered Rings with Two or More Heteroatoms. Handbook of Heterocyclic Chemistry, 3rd ed.; Elsevier Inc.: Amsterdam, The Netherlands, 2010; pp. 498.

6. West, R. C.; Hill, A. F., Advances in Organometallic Chemistry. Elsevier Science: 1995.

7. Lichtenberg, D. W.; Wojcicki, A., Reactions of coordinated propargyl and allene ligands in cyclopentadienyliron dicarbonyl complexes. J. Organomet. Chem. 1975, 94 (2), 311-326. 